\title{
ALGEBRAIC ANALYSIS OF CONTROL SYSTEMS DEFINED BY PARTIAL DIFFERENTIAL EQUATIONS
}

\author{
J.-F. Pommaret \\ CERMICS, Ecole Nationale des Ponts et Chaussées, \\ 6/8 av Blaise Pascal, 77455 Marne-la-Vallée cedex 02, France \\ e-mail: pommaret@cermics.enpc.fr
}

\begin{abstract}
The present chapter contains the material taught within the module P2 of FAP 2004. The purpose of this intensive course is first to provide an introduction to "algebraic analysis". This fashionable though quite difficult domain of pure mathematics today has been pioneered by V.P. Palamodov, M. Kashiwara and B. Malgrange around 1970, after the work of D.C. Spencer on the formal theory of systems of partial differential equations. We shall then focus on its application to control theory in order to study linear control systems defined by partial differential equations with constant or variable coefficients, also called multidimensional control systems, by means of new methods from module theory and homological algebra. We shall revisit a few basic concepts and prove, in particular, that controllability, contrary to a well established engineering tradition or intuition, is an intrinsic structural property of a control system, not depending on the choice of inputs and outputs among the control variables or even on the presentation of the control system. Our exposition will be rather elementary as we shall insist on the main ideas and methods while illustrating them through explicit examples. Meanwhile, we want to stress out the fact that these new techniques bring striking results even on classical control systems of Kalman type !.
\end{abstract}

Key-Words: Algebraic Analysis, Partial Differential Equations, Module Theory, Torsion Module, Projective Module, Projective Equivalence, Homological Algebra, Extension Functor, Control Theory, Controllability, Observability, Characteristic Set.

\section{I.1 INTRODUCTION}

We start recalling and revisiting the important controllability concept in classical control theory. For this, we shall adopt standard notations for the input $u=\left(u^{1}, \ldots, u^{p}\right)$, the state $x=\left(x^{1}, \ldots, x^{n}\right)$ and the output $y=\left(y^{1}, \ldots, y^{m}\right)$, the dot indicating time derivative.

A control system with coefficients in a constant field $k$ (say $\mathbb{Q}, \mathbb{R}, \mathbb{C}$ in general) is said to be in "Kalman form" if it can be written as $\dot{x}=A x+B u$, where $A$ is a constant $n \times n$ matrix and $B$ is a constant $n \times p$ matrix with maximum rank $p$, where we understand that $A$ and $B$ have coefficients in $k$. If we should like to characterize such a system of ordinary differential (OD) equations, we could say that it is linear, first order, with no equation of order zero and with no derivative of $u$ appearing in the OD equations.

Definition 1.1: The above control system is said to be controllable if, starting from any initial point $x_{0}$ at time $t=0$, it is possible to find an input such that the corresponding trajectory is passing through an arbitrary final point $x_{T}$ at a final time $T$.

The following celebrated test has been given by E.R. Kalman in 1963 [6]:

Theorem 1.2: The above control system is controllable if and only if the controllability matrix $\left(B, A B, A^{2} B, \ldots, A^{n-1} B\right)$ has maximum rank $n$.

Of course, in general, starting with $r k(B)=l_{0}=p$, the rank will increase successively and we may set $r k(B, A B)=l_{0}+l_{1}, \ldots, r k\left(B, A B, \ldots, A^{n-1} B\right)=l_{0}+\ldots+l_{n-1}$ as the rank will not increase anymore because of the Cayley-Hamilton theorem saying that $A^{n}$ is a linear combination of the previous lower powers of $A$.

In order to provide a preview of the type of techniques that will be used in the sequel, we provide a new short 
homological proof of the following technical result [25]:

Proposition 1.3: $p=l_{0} \geq l_{1} \geq \ldots \geq l_{n-1} \geq 0$.

Proof: Denoting also by $B$ the vector space over $k$ generated by the column vectors $\left(b_{1}, \ldots, b_{p}\right)$ of $B$, we may introduce the vector spaces $S_{i}=B+A B+\ldots+A^{i} B$ over $k$ for $i=0,1, \ldots, n-1$ and obtain $\operatorname{dim}\left(S_{i} / S_{i-1}\right)=l_{i}$. Denoting also by $A$ the multiplication by $A$, we get $A S_{i}=A B+A^{2} B+\ldots+A^{i+1} B \subseteq S_{i+1}$ and $S_{i+1}=A S_{i}+B$. In this very elementary situation that will be generalized in the sequel, we shall say that a square is "commutative" if the composition of the maps (matrices) along two sides of the square is equal to the composition of the maps along the two other opposite sides, in a standard way. Similarly, a chain of maps will be called a sequence if the composition of two successive maps (matrices) is zero. Accordingly, a sequence (line or column) will be said to be "exact" if the kernel of any map is equal to the image of the preceding map, a " 0 " on the left meaning injectivity (monomorphism) and a "0" on the right meaning surjectivity (epimorphism). We have the following commutative and exact diagram, namely a diagram where all squares are commutative while all lines and columns are exact:

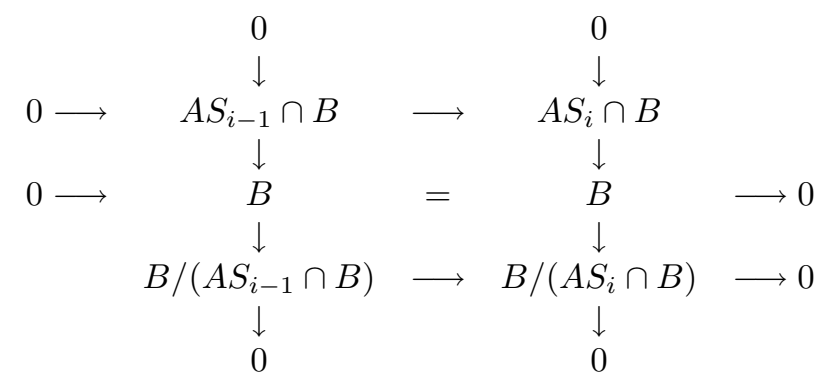

Using the snake theorem (see subsection on homological algebra) or counting the dimensions, we notice that the cokernel $\left(A S_{i} \cap B\right) /\left(A S_{i-1} \cap B\right)$ of the upper map is isomorphic to the kernel of the lower map. We use the lower line of this diagram as the right column of the following commutative and exact diagram:

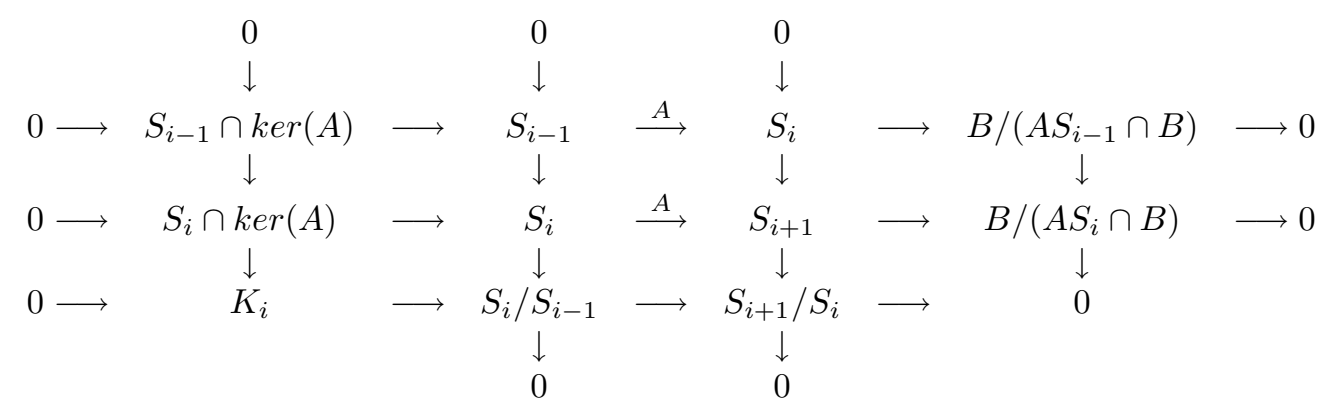

where $K_{i}$ is the kernel of the induced lower central map and we have used the relation $S_{i+1} / A S_{i} \simeq B /\left(A S_{i} \cap B\right)$. Counting the dimensions, we get $l_{i}=l_{i+1}+\operatorname{dim}\left(K_{i}\right) \geq l_{i+1}$. Finally, again using the snake theorem, we get the long exact sequence:

$$
0 \rightarrow S_{i-1} \cap \operatorname{ker}(A) \rightarrow S_{i} \cap \operatorname{ker}(A) \rightarrow K_{i} \rightarrow B /\left(A S_{i-1} \cap B\right) \rightarrow B /\left(A S_{i} \cap B\right) \rightarrow 0
$$

and thus the short exact sequence:

$$
0 \rightarrow\left(S_{i} \cap \operatorname{ker}(A)\right) /\left(S_{i-1} \cap \operatorname{ker}(A)\right) \rightarrow K_{i} \rightarrow\left(A S_{i} \cap B\right) /\left(A S_{i-1} \cap B\right) \rightarrow 0
$$

where we have used the previous isomorphism, as a way to compute $\operatorname{dim}\left(K_{i}\right)$.

Q.E.D.

The reader will have noticed how tricky is such a proof that could be quite tedious otherwise, though we advise the reader to draw pictures of the various spaces involved and their inclusions in order to understand the meaning of the respective quotients.

Surprisingly, through this engineering setting, if we understand that the $l_{i}$, called "controllability indices", can be determined by means of elementary computer algebra (rank of matrices), it seems that we are very far from being able to extend these indices to a more general framework. Indeed, not a single of the previous results can 
be extended to systems of partial differential (PD) equations, or even to systems of OD equations containing the derivatives of the inputs or having variable coefficients. Also, it seems strange that controllability, defined in a purely functional way, could be tested in a purely formal way. Finally, it seems that controllability is highly depending on the choice of inputs and outputs among the control variables, according to a well established engineering tradition.

In order to provide a first feeling that the proper concept of controllability must be revisited, we provide a short but illuminating example:

Example 1.4: Let us consider the system of two OD equations:

$$
\dot{y}^{1}-a y^{2}-\dot{y}^{3}=0 \quad, \quad y^{1}-\dot{y}^{2}+\dot{y}^{3}=0
$$

depending on a constant parameter $a$.

First choosing the control variables to be $y^{1}=x^{1}, y^{2}=x^{2}, y^{3}=u$ and setting $\bar{x}^{1}=x^{1}-u, \bar{x}^{2}=x^{2}-u$, we get the Kalman form:

$$
\dot{\bar{x}}^{1}=a \bar{x}^{2}+a u \quad, \quad \dot{\bar{x}}^{2}=\bar{x}^{1}+u
$$

and the system is controllable, with controlability indices $(1,1)$, if and only if $a \neq 0, a \neq 1$.

Now choosing the control variables to be $y^{1}=x^{1}, y^{2}=u, y^{3}=x^{2}$, and setting anew $\bar{x}^{1}=x^{1}-u, \bar{x}^{2}=x^{2}-u$, though with a totally different meaning, we get the totally different Kalman form:

$$
\dot{\bar{x}}^{1}=-\bar{x}^{1}+(a-1) u \quad, \quad \dot{\bar{x}}^{2}=-\bar{x}^{1}-u
$$

and this new system is controllable, with the same controllability indices, if and only if $a \neq 0, a \neq 1$ too.

It follows from this example that controllability must be a structural property of a control sysem, neither depending on the choice of inputs and outputs among the control variables, nor even on the presentation of the control sytem (change of the control variables eventually leading to change the order of the system). The next definition is crucial for revisiting controllability and extending it to systems of PD equations $[16,18,19]$. It stems from the fact that, in engineering sciences, a measurement apparatus (thermometer, manometer,...) is always measuring a scalar quantity (temperature, pressure,...).

Definition 1.5: An autonomous (or torsion) element is an observable, that is to say a linear combination of the control variables and their derivatives, which satisfies at least one (and thus only one of minimum order when there is only one independent variable) OD or PD equation by itself. An observable satisfying no OD or PD equation for itself will be said to be "free".

This leads to the following formal definition:

Definition 1.6: A control system is controllable if it has no nontrivial autonomous element, that is any observable is free.

Example 1.7: In the preceding example, if $a=0$, setting $z=y^{1}-y^{3}$, we get $\dot{z}=0$. Also, if $a=1$, setting now $z=y^{1}-y^{2}$ and adding the first OD equation to the second, we get $\dot{z}+z=0$.

Though it does not seem evident at first sight, we have:

Proposition 1.8: The preceding Definition is coherent with the Kalman test.

Proof: Using the given OD equations and their derivatives in order to compute $\dot{x}, \ddot{x}, \ldots$ from the arbitrary/parametric $x, u, \dot{u}, \ddot{u}, \ldots$ we could for simplicity imagine that $z=\lambda x+\mu_{0} u+\mu_{1} \dot{u}$ does satisfy the single OD equation $\dot{z}+\nu z=0$. Differentiating $z$ and substituting, we obtain at once the necessary conditions $\mu_{1}=0 \rightarrow \mu_{0}=0$. However, we notice that, if $z$ is an autonomous element, then of course $\dot{z}, \ddot{z}, \ldots$ are again autonomous elements. Hence, from $z=\lambda x$ we successively get $\dot{z}=\lambda A x+\lambda B u \rightarrow \lambda B=0, \ddot{z}=\lambda A^{2} x+\lambda A B u \rightarrow \lambda A B u=0, \ldots$ It follows that there are as many autonomous elements linearly independent over $k$ as the corank of the controllability matrix. Conversely, we notice that, if there exists at least one autonomous element, the control system is surely not controllable in any sense as there is no way to control the OD equation satisfied by this autonomous element. 
However, if a control system is given by high order input/output OD equations, the search for autonomous elements is no longer simple as one cannot use the above computation which is essentially based on the fact that no derivative of the input does appear in the OD equations of the Kalman form.

We now raise another problem.

Ordinary differential $(O D)$ control theory studies input/output relations defined by systems of ordinary differential (OD) equations. In this case, with standard notations, if a control system is defined by input/state/output relations:

$$
\dot{x}=A x+B u \quad, \quad y=C x+D u
$$

with $\operatorname{dim}(x)=n$, this system is "controllable" if $r k\left(B, A B, \ldots, A^{n-1} B\right)=n$, as we already said, and "observable" if $r k\left(\tilde{C}, \tilde{A} \tilde{C}, \ldots, \tilde{A}^{n-1} \tilde{C}\right)=n$ where the tilde sign indicates the transpose of a matrix [25]. Accordingly, the so-called "dual system":

$$
\dot{x}_{a}=-\tilde{A} x_{a}-\tilde{C} u_{a} \quad, \quad y_{a}=\tilde{B} x_{a}+\tilde{D} u_{a}
$$

is controllable (observable) if and only if the given system is observable (controllable). However, and despite many attempts, such a dual definition still seems purely artificial as one cannot avoid introducing the state. The same method could be applied to delay systems with constant coefficients. One must nevertheless notice that, if we do not want to define observability as a property "dual" to controllability, the standard meaning, namely the possibility to recover the state from the input and the output is clear. Indeed, by differentiation, we successively get $y=C x+\ldots, \dot{y}=C A X+\ldots, \ddot{y}=C A^{2} x+\ldots$, and so on, where the missing terms only involve the input and its derivatives.

Hence, if the derivatives of the inputs do appear in the control system, for example in the SISO system $\dot{x}-\dot{u}=0$, not a word is left from the original functional definition of controllability which is only valid for systems in "Kalman form" and the same comment can be made for the corresponding duality.

More generally, "partial differential (PD) control theory" will study input/output relations defined by systems of partial differential (PD) equations. At first sight, we have no longer a way to generalize the Kalman form and not a word of the preceding approach is left as, in most cases, the number of arbitrary parametric derivatives playing the rôle of state could be infinite. However, even if the definition of autonomous elements is still meaningful though we have no longer any way to test it, we also understand that a good definition of controllability and duality should also be valid for control systems with variable coefficients. A similar comment can be made for the definition of the transfer matrix.

Example 1.9: Denoting by $y_{i}^{k}=d_{i} y^{k}$ for $i=1,2$ and $k=1,2,3$ the formal derivatives of the three differential indeterminates $y^{1}, y^{2}, y^{3}$, we consider the system of three PD equations for 3 unknowns and 2 independent variables $[5,18]$ :

$$
\left\{\begin{array}{cccc}
y_{2}^{2} & +y_{2}^{3} & -y_{1}^{3} & -y_{1}^{2}=0 \\
y_{2}^{1} & -y_{2}^{3} & -y_{1}^{3} & -y_{1}^{2}=0 \\
y_{1}^{1} & & -2 y_{1}^{3} & -y_{1}^{2}=0
\end{array}\right.
$$

One can check that one among $\left(y^{1}, y^{2}, y^{3}\right)$ can be given arbitrarily like in the preceding example. Also, setting $z=y^{1}-y^{2}-2 y^{3}$, we get both $z_{1}=0, z_{2}=0$ and $z$ is an autonomous element. Then one can prove that any other autonomous element can be expressible by means of a differential operator acting on $z$ which is therefore a generator (exercise). Accordingly, in the present situation, any autonomous element is a constant multiple of $z$ but no other analogy can be exhibited.

Keeping aside these problems for the moment, let us now turn for a few pages to the formal theory of systems of $\mathrm{OD}$ or $\mathrm{PD}$ equations.

In 1920, M. Janet provided an effective algorithm for looking at the formal (power series) solutions of systems of ordinary differential (OD) or partial differential (PD) equations [5]. The interesting point is that this algorithm also allows to determine the compatibility conditions $\mathcal{D}_{1} \eta=0$ for solving (formally again but this word will not be repeated) inhomogeneous systems of the form $\mathcal{D} \xi=\eta$ when $\mathcal{D}$ is an OD or PD operator and $\xi, \eta$ certain functions. 
Similarly, one can also determine the compatibility conditions $\mathcal{D}_{2} \zeta=0$ for solving $\mathcal{D}_{1} \eta=\zeta$, and so on. With no loss of generality, this construction of a "differential sequence" can be done in such a canonical way that we successively obtain $\mathcal{D}_{1}, \mathcal{D}_{2}, \ldots, \mathcal{D}_{n}$ from $\mathcal{D}$ and $\mathcal{D}_{n}$ is surjective when $n$ is the number of independent variables.

With no reference to the above work, D.C. Spencer developed, from 1965 to 1975, the formal theory of systems of PD equations by relating the preceding results to homological algebra and jet theory [24]. However, this tool has been largely ignored by mathematicians and, "a fortiori", by engineers or even physicists. Therefore, the module theoretic counterpart, today known as "algebraic analysis", which has been pioneered around 1970 by V.P. Palamodov for the constant coefficient case [14], then by M. Kashiwara [7] and B. Malgrange [11] for the variable coefficient case, as it heavily depends on the previous difficult work and looks like even more abstract, has been totally ignored within the range of any application before 1990, when U. Oberst revealed its importance for control theory, in the constant coefficient case only [13].

The purpose of this lecture will essentially be to repare this gap by explaining, in a self-contained way on a few explicit examples, what is the powerfulness of this new approach for understanding both the structural and input/output properties of linear PD control systems, also called multidimensional or simply n-dimensional. Meanwhile, the reader will evaluate the price to pay for such a better understanding. Needless to say that many results obtained could not even be imagined without this new approach, dating back to 1986 when we gave for the first time the formal definition of controllability of a control system [16] but now largely acknowledged by the control community [26,27].

\section{I.2 MOTIVATING EXAMPLES}

As we always use to say, the difficulty in studying systems of PD equations is not only of a functional nature (unicity and existence of solutions,...) but also of a formal nature (integrability and compatibility conditions,...). This is the reason for which the study of algebraic analysis is at once touching delicate points of differential geometry, the main one being formal integrability. Hence, forgetting about control theory for a few pages, we now explain this concept and other related ones on a few tricky motivating examples. It will therefore be a first striking challenge for the reader to wonder what certain of these academic/engineering examples have to do with controllability!.

Motivating Example 2.1: With two independent variables $\left(x^{1}, x^{2}\right)$, one unknown $y$ and standard notations for PD equations and computer algebra (MACSYMA, MAPLE, MATHEMATICA,...), we consider the following third order system of $\mathrm{PD}$ equations with second member $(u, v)$ :

$$
\left\{\begin{array}{l}
P y \equiv d_{222} y+x^{2} y=u \\
Q y \equiv d_{2} y+d_{1} y=v
\end{array}\right.
$$

where $P$ and $Q$ are PD operators with coefficients in the (differential) field $K=\mathbb{Q}\left(x^{1}, x^{2}\right)$ of rational functions in $x^{1}$ and $x^{2}$. We check the identity $Q P-P Q \equiv 1$ and obtain easily:

$$
y=Q u-P v=d_{2} u+d_{1} u-d_{222} v-x^{2} v
$$

Substituting in the previous PD equations, we therefore obtain the two generating $6^{\text {th }}$-order compatibility conditions for $(u, v)$ in the form:

$$
\left\{\begin{array}{l}
A \equiv P Q u-P^{2} v-u=0 \\
B \equiv Q^{2} u-Q P v-v=0
\end{array}\right.
$$

These two compatibility conditions are not differentially independent as we check at once:

$$
\begin{aligned}
Q A & \equiv Q P Q u-Q P^{2} v-Q u \\
& \equiv(1+P Q) Q u-(1+P Q) P v-Q u \\
& \equiv P Q^{2} u-P Q P v-P v \\
& \equiv P B
\end{aligned}
$$

Finally, setting $u=0, v=0$, we notice that the preceding homogeneous system can be written in the form $\mathcal{D} y=0$ and admits the only solution $y=0$. 
Motivating Example 2.2: Again with two independent variables $\left(x^{1}, x^{2}\right)$ and one unknown $y$, let us consider the following second order system with constant coefficients:

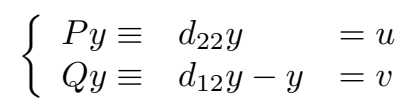

where now $P$ and $Q$ are PD operators with coefficients in the subfield $k=\mathbb{Q}$ of constants of $K$. We obtain at once:

$$
y=d_{11} u-d_{12} v-v
$$

and could hope to obtain the $4^{t h}$-order generating compatibility conditions by substitution, that is to say:

$$
\begin{cases}A \equiv d_{1122} u-d_{1222} v-d_{22} v-u & =0 \\ B \equiv d_{1112} u-d_{11} u-d_{1122} v & =0\end{cases}
$$

However, in this particular case, we notice that there is an unexpected unique second order generating compatibility condition of the form:

$$
C \equiv d_{12} u-u-d_{22} v=0
$$

as we now have indeed $P Q-Q P=0$ both with $A \equiv d_{12} C+C$ and $B \equiv d_{11} C$, a result leading to $C \equiv$ $d_{22} B-d_{12} A+A$. Accordingly, the systems $A=0, B=0$ on one side and $C=0$ on the other side are completely different though they have the same solutions in $u, v$.

Finally, setting $u=0, v=0$, we notice that the preceding homogeneous system can be written in the form $\mathcal{D} y=0$ and admits the only solution $y=0$, like the preceding example.

Remark 2.3: It is only in the last section of this chapter that we shall understand why such a specific result could only happen for systems of PD equations with constant coefficients. Indeed, this result will be shown to be a straightforward consequence of the famous Quillen-Suslin theorem which can be considered as one of the most difficult theorems of mathematics [23].

Motivating Example 2.4: (See [18] for more details on this interesting example first provided by M. Janet in [5]). With $n=3$, let us consider the second order system:

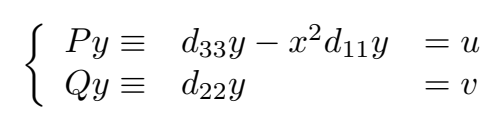

where now $P$ and $Q$ are PD operators with coefficients in the (differential) field $K=\mathbb{Q}\left(x^{1}, x^{2}, x^{3}\right)$. Introducing as before:

$$
d_{112} y=\frac{1}{2}\left(d_{33} v-x^{2} d_{11} v-d_{22} u\right)=w
$$

we finally get the two following compatibility conditions:

$$
\begin{cases}A \equiv d_{233} v-x^{2} d_{112} v-3 d_{11} v-d_{222} u & =0 \\ B \equiv d_{3333} w-2 x^{2} d_{1133} w+\left(x^{2}\right)^{2} d_{1111} w-d_{11233} u+x^{2} d_{11112} u-d_{1111} u & =0\end{cases}
$$

These two compatibility conditions of respective orders 3 and 6 are differentially dependent as one checks at once through computer algebra:

$$
d_{3333} A-2 x^{2} d_{1133} A+\left(x^{2}\right)^{2} d_{1111} A-2 d_{2} B=0
$$

However, and contrary to the two preceding motivating examples, we now have no way to know whether $A$ and $B$ are the only two generating compatibility conditions.

Finally, in the present situation, the space of solutions of the system $\mathcal{D} y=0$ can be seen to be expressible by polynomials in $\left(x^{1}, x^{2}, x^{3}\right)$ and has dimension 12 over the constants.

Having in mind the preceding examples and a computer algebra framework, we are in a position to explain in a simple manner how systems of PD equations can be divided into two classes, namely the "good" ones and the "bad" ones. This terminology will lead, in the next section, to an intrinsic/coordinate-free formulation where the good systems will be called "formally integrable" systems.

For understanding the difference, let us plug a given system of order $q$ and use computer algebra to have all possible information about the various derivatives of the unknowns at the orders $q+1, q+2, \ldots$, by differentiating the given $\mathrm{PD}$ equations successively once, twice, ... and so on. In the case of the first example with 
$q=3$, differentiating once we get the two new second order PD equation $d_{12} y+d_{11} y=0, d_{22} y+d_{12} y=0$. Similarly, differentiating twice we get four linearly independent PD equations of strict order three, namely $d_{222} y+x^{2} y=0, d_{122} y-x^{2} y=0, d_{112} y+x^{2} y=0, d_{111} y-x^{2} y=0$ though we had only one initially. Finally, differentiating three times, we even get the zero order equation $y=0$, that we need again to differentiate once in order to get separately $d_{1} y=0, d_{2} y=0$, and so on. In the case of the second example, the things are similar and we understand that, in general, we have to differentiate $r+s(r)$ times in order to know all the possible information on all the derivatives up to order $q+r$. The situation is even more tricky in the case of the third example as, differentiating once with respect to $x^{1}, x^{2}, x^{3}$ respectively, we get 6 linearly independent PD equations of strict order 3, with no information "backwards" on the order 2 as in the preceding situations. However, we are not finished with the order 3 as indeed, differentiating twice, that is once more, we get the new equation $d_{112} y=0$ but we are not sure that,differentiating 100 times will not produce new equations. In fact, in order to be convinced about the difficulty of this problem and how highly it depends on the coefficients, we ask the reader, as an exercise, to work out this example with $\left(x^{2}\right)^{r}$ in place of $x^{2}$ for $r=1,2,3, \ldots$ successively.

It is essential to notice that, contrary to the use of Gröbner bases, the preceding results do not depend on any change of both the independent or the dependent variables among them as the order is unchanged.

Contrary to the previous (very) bad examples, in the case of good examples, differentiating just $r$ times will provide all information on the derivatives up to order $q+r$.

Example 2.5: With all indices ranging from 1 to $n$, let us consider the so-called Killing system:

$$
\frac{1}{2}(\mathcal{L}(\xi) \omega)_{i j} \equiv \frac{1}{2}\left(\omega_{r j} \partial_{i} \xi^{r}+\omega_{i r} \partial_{j} \xi^{r}+\xi^{r} \partial_{r} \omega_{i j}\right)=\epsilon_{i j}
$$

where $\omega_{i j}=1$ if $i=j$ and 0 if $i \neq j$ and we have used the standard Einstein implicit summation on the up and down indices. This system is used in continuum mechanics to define the (small) strain tensor $\epsilon$ from the displacement vector $\xi$ by using the Lie derivative of the euclidean metric $\omega$ along the vector field $\xi$. If we look for a displacement providing no strain, we have just to set $\epsilon=0$ and to notice (tricky exercise !) that differentiating once provides the $n^{2}(n+1) / 2$ second order PD equations $d_{i j} \xi^{k}=0$ that can be easily integrated. Hence we get $\xi^{k}=A_{r}^{k} x^{r}+B^{k}$ with $A$ an arbitrary skew-symmetric constant matrix and $B$ an arbitrary vector, that is to say we recognize an arbitrary infinitesimal rigid motion. This is a good system indeed as the $n+1$ derivatives up to order one are arbitrary, the $n(n+1) / 2$ given equations provide all information on the $n^{2}$ derivatives of strict order one and all the other derivatives of order $\geq 2$ vanish.

More generally, any linear system with constant coefficients having only derivatives of the same order appearing in it is automatically good.

In order to look for the compatibility conditions for $\epsilon$, we notice that the compatibilty conditions of order $r$ are obtained by Cramer's rule through the elimination of the $n(n+r) ! /(n-1) !(r+1)$ ! derivatives of strict order $r+1$ of the $\xi$ from the $n(n+1)(n+r-1) ! / 2(n-1) ! r$ ! derivatives of strict order $r$ of the $\epsilon$. Accordingly, we get no compatibility condition of order one and $n^{2}(n+1)^{2} / 4-n^{2}(n+1)(n+2) / 6=n^{2}\left(n^{2}-1\right) / 12$ compatibility conditions of order two. For the case $n=2$ of 2 -dimensional plane elasticity, we get the only second order compatibility condition :

that will be used in the sequel.

$$
\partial_{11} \epsilon_{22}+\partial_{22} \epsilon_{11}-2 \partial_{12} \epsilon_{12}=0
$$

We now explain the close relationship existing between the search for compatibility conditions and the study of autonomous elements. Indeed, in order to look for the self-governing PD equations of a given autonomous element, we just need to write out the system of PD equations with zero second member, add the expression of the autonomous element as a left member/operator, keeping the autonomous element as a right member and look for the set of compatibility conditions of the total system thus obtained.

Examp 2.6: With respect to the system:

$$
d_{22} y=0 \quad, \quad d_{12} y=0
$$

if we add $d_{1} y=z$ we get $d_{2} z=0$ while if we add $d_{2} y=z$, we get $d_{2} z=0, d_{1} z=0$.

This result proves that, contrary to the OD case where a given autonomous element always satisfies a single generating $\mathrm{OD}$ equation, in the PD case the situation can be far more complicate. In fact, there is a way to classify autonomous elements according to the type of system they satisfy. This result, leading to the concept of "purity", 
involves a lot of delicate homological algebra and is out of the scope of this chapter. For more details, we invite the interested reader to look at [19, section V.1.3]

In view of the importance of autonomous elements in differential algebra [16,18], we first notice that the corresponding concept can be easily extended to nonlinear systems and we have the following proposition:

Proposition 2.7: The sum, product, quotient and derivatives of two autonomous elements are again autonomous elements.

Proof: We only prove this result for the sum as the other proofs are similar. As an autonomous element satisfies at least one OD or PD equation of order $q$ for itself, the number of its arbitrary/parametric derivatives up to order $q+r$ is at most equal to:

$$
(n+q+r) ! / n !(q+r) !-(n+r) ! / n ! r !=\frac{q}{(n-1) !} r^{n-1}+\ldots
$$

and this number is multiplied by 2 in the sum while the number of derivatives of the sum up to order $r$ is $(n+r) ! / n ! r !=\frac{1}{n !} r^{n}+\ldots$ Accordingly, when $r$ is large enough, this last number is greater than the preceding number and the sum cannot be free.

Q.E.D.

We end this section presenting two examples where any observable is autonomous.

Example 2.8: (More details concerning the Bénard problem can be found in [18,p 366]). When a viscous liquid is in between two horizontal parallel plates with distance $L$ between them, the lower heated one being at temperature $T_{0}$ while the upper one is at temperature $T_{1}$ with $T_{0}-T_{1}=A g L>0$ where $\vec{g}$ is the vertical gravity, its stationary evolution is governed by the following linear Boussinesq system of five PD equations:

$$
\begin{gathered}
\vec{\nabla} \cdot \vec{v}=0 \\
\eta \Delta \vec{v}-\vec{\nabla} \pi-\alpha a \theta \vec{g}=0 \\
\lambda \Delta \theta-\frac{A}{g} \vec{g} \cdot \vec{v}=0
\end{gathered}
$$

describing successively the continuity equation, the three Navier-Stokes equations and the heat equation. In this system, the mass per unit volume is $\rho=a\left(1-\alpha\left(T-T_{0}\right)\right)$ with $a=\rho\left(T_{0}\right), \eta$ is the viscosity coefficient, $\pi$ and $\theta$ are the respective perturbations of the pressure and temperarure around an equilibrium state and $\lambda=\kappa a c$ where $\kappa$ is the thermal conductivity and $c$ is the heating coefficient at constant pressure and temperature $T_{0}$.

We prove that $\theta$ is an autonomous element.

Taking into account the continuity equation while remembering a well known vector identity, we obtain at once:

$$
\vec{\nabla} \wedge \vec{\nabla} \wedge \vec{v}=\vec{\nabla}(\vec{\nabla} \cdot \vec{v})-\Delta \vec{v}=-\Delta \vec{v}
$$

Now, applying twice the curl operator to the Navier-Stokes equations for eliminating $\pi$ and projecting onto the vertical axis $x^{3}$, we get:

$$
\eta \Delta \Delta v^{3}+\alpha a g\left(d_{11}+d_{22}\right) \theta=0
$$

Using the heat equation in the form $\lambda \Delta \theta=A v^{3}$ and introducing the dimensionless Rayleigh number $R=$ $g \alpha A L^{4} a^{2} c / \eta \kappa$, we finally obtain the following sixth order PD equation:

$$
\Delta \Delta \Delta \theta-\frac{R}{L^{4}}\left(d_{11}+d_{22}\right) \theta=0
$$

The same equation is satisfied by $v^{3}$ but we notice that the vertical component $\zeta=d_{1} v^{2}-d_{2} v^{1}$ only satisfies $\Delta \zeta=0$.

Example 2.9: The Euler equations for an incompressible fluid with speed $\vec{v}$, pressure $p$ and mass per unit volume set to 1 , are made by the nonlinear system:

$$
\vec{\nabla} \cdot \vec{v}=0 \quad, \quad d_{t} \vec{v}+(\vec{v} \cdot \vec{\nabla}) \vec{v}+\vec{\nabla} p=0
$$


For a 1-dimensional flow, we get $d_{x} v=0, d_{t} v+v d_{x} v+d_{x} p=0$ and thus both $v$ and $p$ are autonomous because $d_{x} v=0, d_{x x} p=0$. For a 2-dimensional flow, $v^{1}$ is autonomous but its highly nonlinear fifth order decoupling PD equation is covering one full page of book [19,p 40].

We end this section with a nonlinear example, showing out that the study of linear systems can be of some help for studying the structural properties of nonlinear systems by means of linearization, only if we are able to deal with linear systems with variable coefficients.

Example 2.10: Let us consider the single input/single output (SISO) system $u \dot{y}-\dot{u}=a=c s t$ and ask about its controllability (See [18] for more details). Of course, if $a=0$, setting $z=y-\log u$, we get $\dot{z}=0$ and the system cannot be controlled as there is one autonomous element. Introducing the variations $U=\delta u, Y=\delta y$, the generic linearization (not to be confused with the linearization at a specific solution) becomes:

$$
u \dot{Y}-\dot{U}+\dot{y} U=0
$$

as the constant parameter $a$ is untouched. It seems that this system is no longer depending on $a$ but the reader must not forget that $u=u(t)$ and $y=y(t)$ are solutions of the given nonlinear system which definitively depends on $a$. According to the previous comments, it just remains to study under what condition on $a$ the above linear system with variable coefficients is controllable. The use of such a study is as follows. If the linearized system is controlabe, the nonlinear system is surely controllable because, otherwise, it should have at least one autonomous element, the linearization of which should satisfy the corresponding linearized decoupling equation. The converse is not evident as an autonomous element for the linearized system may not necessarily come from the linearization of an autonomous element for the nonlinear system. In fact, one can prove that that this converse is only true for OD systems but false in general for PD systems as counterexamples may exist [19].

In the present example, when $a=0$, we easily check that $\delta z=Z=Y-\frac{1}{u} U$ satisfies $\dot{Z}=0$ and the problem is thus to obtain this critical value $a=0$ directly from the linear system, a result highly not evident at first sight, even on this elementary example.

\section{I.3 ALGEBRAIC ANALYSIS}

It becomes clear from the examples of the second section that there is a need for classifying the properties of systems of $\mathrm{PD}$ equations in a way that does not depend on their presentations and this is the purpose of algebraic analysis.

\section{I.3.1 Module Theory}

Before entering the heart of this chapter, we need a few technical definitions and results from commutative algebra $[4,8,12,23]$.

First of all, we start defining rings and modules.

Definition 3.1.1: A ring $A$ is a non-empty set with two associative binary operations called addition and $m u$ ultiplication, respectively sending $a, b \in A$ to $a+b \in A$ and $a b \in A$ in such a way that $A$ becomes an abelian group for the multiplication, so that $A$ has a zero element denoted by 0 , every $a \in A$ has an additive inverse denoted by $-a$ and the multiplication is distributive over the addition, that is to say $a(b+c)=a b+a c,(a+b) c=a c+b c, \forall a, b, c \in A$. A $\operatorname{ring} A$ is said to be unitary if it has a (unique) element $1 \in A$ such that $1 a=a 1=a, \forall a \in A$ and commutative if $a b=b a, \forall a, b \in A$.

A non-zero element $a \in A$ is called a zero-divisor if one can find a non-zero $b \in A$ such that $a b=0$ and a ring is called an integral domain if it has no zero-divisor.

Definition 3.1.2: A ring $K$ is called a field if every non-zero element $a \in K$ is a unit, that is one can find an element $b \in K$ such that $a b=1 \in K$.

Definition 3.1.3: A module $M$ over a ring $A$ or simply an $A$-module is a set of elements $x, y, z, \ldots$ which is an abelian group for an addition $(x, y) \rightarrow x+y$ with an action $A \times M \rightarrow M:(a, x) \rightarrow a x$ satisfying:

- $\quad a(x+y)=a x+a y, \forall a \in A, \forall x, y \in M$

- $\quad a(b x)=(a b) x, \forall a, b \in A, \forall x \in M$

- $\quad(a+b) x=a x+b x, \forall a, b \in A, \forall x \in M$

- $1 x=x, \forall x \in M$ 
The set of modules over a ring $A$ will be denoted by $\bmod (A)$. A module over a field is called a vector space.

Definition 3.1.4: A map $f: M \rightarrow N$ between two $A$-modules is called a homomorphism over $A$ if $f(x+y)=$ $f(x)+f(y), \forall x, y \in M$ and $f(a x)=a f(x), \forall a \in A, \forall x \in M$. We successively define:

$$
\begin{array}{ll}
\bullet & \operatorname{ker}(f)=\{x \in M \mid f(x)=0\} \\
\bullet & \operatorname{im}(f)=\{y \in N \mid \exists x \in M, f(x)=y\} \\
\text { - } & \operatorname{coker}(f)=N / \operatorname{im}(f)
\end{array}
$$

Definition 3.1.5: We say that a chain of modules and homomorphisms is a sequence if the composition of two successive such homomorphisms is zero. A sequence is said to be exact if the kernel of each map is equal to the image of the map preceding it. An injective homomorphism is called a monomorphism, a surjective homomorphism is called an epimorphism and a bijective homomorphism is called an isomorphism. A short exact sequence is an exact sequence made by a monomorphism followed by an epimorphism.

The proof of the following proposition is left to the reader as an exercise:

Proposition 3.1.6: If one has a short exact sequence:

$$
0 \longrightarrow M^{\prime} \stackrel{f}{\longrightarrow} M \stackrel{g}{\longrightarrow} M^{\prime \prime} \longrightarrow 0
$$

then the following conditions are equivalent:

- There exists a monomorphism $v: M^{\prime \prime} \rightarrow M$ such that $g \circ v=i d_{M^{\prime \prime}}$.

- There exists an epimorphism $u: M \rightarrow M^{\prime}$ such that $u \circ f=i d_{M^{\prime}}$.

- There exist isomorphisms $\varphi=(u, g): M \rightarrow M^{\prime} \oplus M^{\prime \prime}$ and $\psi=f+v: M^{\prime} \oplus M^{\prime \prime} \rightarrow M$ that are inverse to each other and provide an isomorphism $M \simeq M^{\prime} \oplus M^{\prime \prime}$

Definition 3.1.7: In the above situation, we say that the short exact sequence splits and $u(v)$ is called a lift for $f(g)$. In particular we have the relation: $f \circ u+v \circ g=i d_{M}$.

Definition 3.1.8: A left (right) ideal $\mathfrak{a}$ in a $\operatorname{ring} A$ is a submodule of $A$ considered as a left (right) module over itself. When the inclusion $\mathfrak{a} \subset A$ is strict, we say that $\mathfrak{a}$ is a proper ideal of $A$.

Lemma 3.1.9: If $\mathfrak{a}$ is an ideal in a ring $A$, the set of elements $\operatorname{rad}(\mathfrak{a})=\left\{a \in A \mid \exists n \in \mathbb{N}, a^{n} \in \mathfrak{a}\right\}$ is an ideal of $A$ containing $\mathfrak{a}$ and called the radical of $\mathfrak{a}$. An ideal is called perfect or radical if it is equal to its radical.

Definition 3.1.10: For any subset $S \subset A$, the smallest ideal containing $S$ is called the ideal generated by $S$. An ideal generated by a single element is called a principal ideal and a ring is called a principal ideal ring if any ideal is principal. The simplest example is that of polynomial rings in one indeterminate over a field. When $\mathfrak{a}$ and $\mathfrak{b}$ are two ideals of $A$, we shall denote by $\mathfrak{a}+\mathfrak{b}(\mathfrak{a} \mathfrak{b})$ the ideal generated by all the sums $a+b$ (products $a b$ ) with $a \in \mathfrak{a}, b \in \mathfrak{b}$.

Definition 3.1.11: An ideal $\mathfrak{p}$ of a ring $A$ is called a prime ideal if, whenever $a b \in \mathfrak{p}(a A b \in \mathfrak{p}$ in the noncommutative case) then either $a \in \mathfrak{p}$ or $b \in \mathfrak{p}$. The set of proper prime ideals of $A$ is denoted by $\operatorname{spec}(A)$ and called the spectrum of $A$.

Definition 3.1.12: The annihilator of a module $M$ in $A$ is the ideal $a n n_{A}(M)$ of $A$ made by all the elements $a \in A$ such that $a x=0, \forall x \in M$.

From now on, all rings considered will be unitary integral domains, that is rings containing 1 and having no zero-divisor. For the sake of clarity, as a few results will also be valid for modules over non-commutative rings, we shall denote by ${ }_{A} M_{B}$ a module $M$ which is a left module for $A$ with operation $(a, x) \rightarrow a x$ and a right module for $B$ with operation $(x, b) \rightarrow x b$. In the commutative case, lower indices are not needed. If $M={ }_{A} M$ and $N={ }_{A} N$ are two left $A$-modules, the set of $A$-linear maps $f: M \rightarrow N$ will be denoted by $h_{o m}(M, N)$ or simply $h o m(M, N)$ when there will be no confusion and there is a canonical isomorphism $h o m(A, M) \simeq M: f \rightarrow f(1)$ with inverse $x \rightarrow(a \rightarrow a x)$. When $A$ is commutative, $\operatorname{hom}(M, N)$ is again an $A$-module for the law $(b f)(x)=f(b x)$ as we have indeed:

$$
(b f)(a x)=f(b a x)=f(a b x)=a f(b x)=a(b f)(x) .
$$


In the non-commutative case, things are much more complicate and we have:

Lemma 3.1.13: Given ${ }_{A} M_{B}$ and ${ }_{A} N$, then $\operatorname{hom}_{A}(M, N)$ becomes a left module over $B$ for the law $(b f)(x)=f(x b)$.

Proof: We just need to check the two relations:

$$
\begin{gathered}
(b f)(a x)=f(a x b)=a f(x b)=a(b f)(x), \\
\left(b^{\prime}\left(b^{\prime \prime} f\right)\right)(x)=\left(b^{\prime \prime} f\right)\left(x b^{\prime}\right)=f\left(x b^{\prime} b^{\prime \prime}\right)=\left(\left(b^{\prime} b^{\prime \prime}\right) f\right)(x) .
\end{gathered}
$$

Q.E.D.

A similar result can be obtained (exercise) with ${ }_{A} M$ and ${ }_{A} N_{B}$, where $\operatorname{hom}_{A}(M, N)$ now becomes a right $B$ module for the law $(f b)(x)=f(x) b$.

Now we recall that a sequence of modules and maps is exact if the kernel of any map is equal to the image of the map preceding it and we have:

Theorem 3.1.14: If $M, M^{\prime}, M^{\prime \prime}$ are $A$-modules, the sequence:

$$
M^{\prime} \stackrel{f}{\rightarrow} M \stackrel{g}{\rightarrow} M^{\prime \prime} \rightarrow 0
$$

is exact if and only if the sequence:

$$
0 \rightarrow \operatorname{hom}\left(M^{\prime \prime}, N\right) \rightarrow \operatorname{hom}(M, N) \rightarrow \operatorname{hom}\left(M^{\prime}, N\right)
$$

is exact for any $A$-module $N$.

Proof: Let us consider homomorphisms $h: M \rightarrow N, h^{\prime}: M^{\prime} \rightarrow N, h^{\prime \prime}: M^{\prime \prime} \rightarrow N$ such that $h^{\prime \prime} \circ g=h, h \circ f=h^{\prime}$. If $h=0$, then $h^{\prime \prime} \circ g=0$ implies $h^{\prime \prime}\left(x^{\prime \prime}\right)=0, \forall x^{\prime \prime} \in M^{\prime \prime}$ because $g$ is surjective and we can find $x \in M$ such that $x^{\prime \prime}=g(x)$. Then $h^{\prime \prime}\left(x^{\prime \prime}\right)=h^{\prime \prime}(g(x))=h^{\prime \prime} \circ g(x)=0$. Now, if $h^{\prime}=0$, we have $h \circ f=0$ and $h$ factors through $g$ because the initial sequence is exact. Hence there exists $h^{\prime \prime}: M^{\prime \prime} \rightarrow N$ such that $h=h^{\prime \prime} \circ g$ and the second sequence is exact.

We let the reader prove the converse as an exercise.

Q.E.D.

Similarly, one can prove (exercise):

Corollary 3.1.15: The short exact sequence:

$$
0 \rightarrow M^{\prime} \rightarrow M \rightarrow M^{\prime \prime} \rightarrow 0
$$

splits if and only if the short exact sequence:

$$
0 \rightarrow \operatorname{hom}\left(M^{\prime \prime}, N\right) \rightarrow \operatorname{hom}(M, N) \rightarrow \operatorname{hom}\left(M^{\prime}, N\right) \rightarrow 0
$$

is exact for any module $N$.

Definition 3.1.16: If $M$ is a module over a $\operatorname{ring} A$, a system of generators of $M$ over $A$ is a family $\left\{x_{i}\right\}_{i \in I}$ of elements of $M$ such that any element of $M$ can be written $x=\sum_{i \in I} a_{i} x_{i}$ with only a finite number of nonzero $a_{i}$.

Definition 3.1.17: An $A$-module is called noetherian if every submodule of $M$ (and thus $M$ itself) is finitely generated.

One has the following technical lemma (exercise):

Lemma 3.1.18: In a short exact sequence of modules, the central module is noetherian if and only if the two other modules are noetherian. 
We obtain in particular:

Proposition 3.1.19: If $A$ is a noetherian ring and $M$ is a finitely generated module over $A$, then $M$ is noetherian.

Proof: Applying the lemma to the short exact sequence $0 \rightarrow A^{r-1} \rightarrow A^{r} \rightarrow A \rightarrow 0$ where the epimorphism on the right is the projection onto the first factor, we deduce by induction that $A^{r}$ is noetherian. Now, if $M$ is generated by $\left\{x_{1}, \ldots, x_{r}\right\}$, there is an epimorphism $A^{r} \rightarrow M:(1,0, \ldots, 0) \rightarrow x_{1}, \ldots,\{0, \ldots, 0,1\} \rightarrow x_{r}$ and $M$ is noetherian because of the lemma.

Q.E.D.

In the preceding situation, the kernel of the epimorphism $A^{r} \rightarrow M$ is also finitely generated, say by $\left\{y_{1}, \ldots, y_{s}\right\}$ and we therefore obtain the exact sequence $A^{s} \rightarrow A^{r} \rightarrow M \rightarrow 0$ that can be extended inductively to the left.

Definition 3.1.20: In this case, we say that $M$ is finitely presented.

We now present the technique of localization that will play a central part and is used to introduce rings and modules of fractions. We shall define the procedure in the non-commutative case but the reader will discover that, in the commutative case, localization is just the formal counterpart superseding Laplace transform as there is no longer any technical assumption on the initial data. Indeed, it is well known that, if the Laplace transform is $f(t) \rightarrow \hat{f}(s)=\int_{0}^{\infty} e^{-s t} f(t) d t$, then $d f(t) / d t \rightarrow s \hat{f}(s)$ only if we suppose that $f(0)=0$. Of course, the achievement of introducing rational functions through the transfer matrix is the heart of the procedure which is also valid in the multidimensional (PD) case. However, it is essential to notice that only the localization technique can be applied to systems with variable coefficients.

We start with a basic definition:

Definition 3.1.21: A subset $S$ of a $\operatorname{ring} A$ is said to be multiplicatively closed if $\forall s, t \in S \Rightarrow s t \in S$ and $1 \in S$.

Example 3.1.22: We provide a few useful cases:

- $\forall a \in A$, we may consider $S_{a}=\left\{1, a, a^{2}, \ldots\right\}$.

- $\forall \mathfrak{p} \in \operatorname{spec}(A)$, we may consider $S=A-\mathfrak{p}$.

- For any ideal $\mathfrak{a} \in A$, we may consider $S=\{1+a \mid a \in \mathfrak{a}\}$.

- We may consider the set $S$ of non-zerodivisors of $A$. In particular, if $A$ is an integral domain, we may consider $S=A-\{0\}$.

In a general way, whenever $A$ is a non-commutative ring, that is $a b \neq b a$ when $a, b \in A$, we shall set the following definition:

Definition 3.1.23: By a left ring of fractions or left localization of a noncommutative ring $A$ with respect to a multiplicatively closed subset $S$ of $A$, we mean a ring denoted by $S^{-1} A$ and a homomorphism $\theta=\theta_{S}: A \rightarrow S^{-1} A$ such that:

1) $\theta(s)$ is invertible in $S^{-1} A, \forall s \in S$.

2) Each element of $S^{-1} A$ or fraction has the form $\theta(s)^{-1} \theta(a)$ for some $s \in S, a \in A$.

3) $\operatorname{ker}(\theta)=\{a \in A \mid \exists s \in S, s a=0\}$.

A right ring of fractions or right localization can be similarly defined.

In actual practice, a fraction will be simply written $s^{-1} a$ and we have to distinguish carefully $s^{-1} a$ from $a s^{-1}$. We shall recover the standard notation $a / s$ of the commutative case when two fractions $a / s$ and $b / t$ can be reduced to the same denominator $s t=t s$. The following proposition is essential and will be completed by two technical lemmas that will be used for constructing localizations.

Proposition 3.1.24: If there exists a left localization of $A$ with respect to $S$, then we must have:

1) $S a \cap A s \neq 0, \forall a \in A, \forall s \in S$.

2) If $s \in S$ and $a \in A$ are such that $a s=0$, then there exists $t \in S$ such that $t a=0$.

Proof: The element $\theta(a) \theta(s)^{-1}$ in $S^{-1} A$ must be of the form $\theta(t)^{-1} \theta(b)$ for some $t \in S, b \in A$. Accordingly, $\theta(a) \theta(s)^{-1}=\theta(t)^{-1} \theta(b) \Rightarrow \theta(t) \theta(a)=\theta(b) \theta(s)$ and thus $\theta(t a-b s)=0 \Rightarrow \exists u \in S, u t a=u b s$ with $u t \in S, u b \in A$. 
Finally, as $=0 \Rightarrow \theta(a) \theta(s)=0 \Rightarrow \theta(a)=0$ because $\theta(s)$ is invertible in $S^{-1} A$. Hence $\exists t \in S$ such that $t a=0$.

Q.E.D.

Definition 3.1.25: A set $S$ satisfying the condition 1) is called a left Ore set.

Lemma 3.1.26: If $S$ is a left Ore set in a noetherian ring, then $S$ also satisfies the condition 2) of the preceding lemma.

Proof: Let $s \in S$ and $a \in A$ be such that $a s=0$. Denoting the left annihilator by $\operatorname{lann}$, we have $l a n n\left(s^{n}\right) \subseteq$ $\operatorname{lann}\left(s^{n+1}\right)$ for each integer $n$. As $A$ is noetherian, $\operatorname{lann}\left(s^{n}\right)=\operatorname{lann}\left(s^{n+1}\right)$ for $n \gg 0$. Using the left Ore condition, we can find $t \in S, b \in A$ such that $t a=b s^{n}$ and thus $b s^{n+1}=\operatorname{tas}=0 \Rightarrow b \in \operatorname{lann}\left(s^{n+1}\right)=\operatorname{lann}\left(s^{n}\right) \Rightarrow \operatorname{ta}=0$. We notice that $S^{-1} A$ is the zero ring if and only if $0 \in S$ and the second condition is not needed when $A$ is an integral domain.

Q.E.D.

Lemma 3.1.27: If $S$ is a left Ore set in a ring $A$, then $A s \cap A t \cap S \neq 0, \forall s, t \in S$ and two fractions can be brought to the same denominator.

Proof: From the left Ore condition, we can find $u \in S$ and $a \in A$ such that $u s=a t \in S$. More generally, we can find $u, v \in A$ such that $u s=v t \in S$ and we successively get:

$$
\begin{gathered}
\theta(u s)^{-1} \theta(u a)=\theta(s)^{-1} \theta(u)^{-1} \theta(u) \theta(a)=\theta(s)^{-1} \theta(a), \forall a \in A \\
\theta(v t)^{-1} \theta(v b)=\theta(t)^{-1} \theta(v)^{-1} \theta(v) \theta(b)=\theta(t)^{-1} \theta(b)
\end{gathered}
$$

so that the two fractions $\theta(s)^{-1} \theta(a)$ and $\theta(t)^{-1} \theta(b)$ can be brought to the same denominator $\theta(u s)=\theta(v t)$.

Q.E.D.

We are now in position to construct the ring of fractions $S^{-1} A$ whenever $S$ satifies the two conditions of the last proposition. For this, using the preceding lemmas, let us define an equivalence relation on $S \times A$ by saying that $(s, a) \sim(t, b)$ if one can find $u, v \in S$ such that $u s=v t \in S$ and $u a=v b$. Such a relation is clearly reflexive and symmetric, thus we only need to prove that it is transitive. So let $\left(s_{1}, a_{1}\right) \sim\left(s_{2}, a_{2}\right)$ and $\left(s_{2}, a_{2}\right) \sim\left(s_{3}, a_{3}\right)$. Then we can find $u_{1}, u_{2} \in A$ such that $u_{1} s_{1}=u_{2} s_{2} \in S$ and $u_{1} a_{1}=u_{2} a_{2}$. Also we can find $v_{2}, v_{3} \in A$ such that $v_{2} s_{2}=v_{3} s_{3} \in S$ and $v_{2} a=v_{3} a_{3}$. Now, from the Ore condition, one can find $w_{1}, w_{3} \in A$ such that $w_{1} u_{1} s_{1}=w_{3} v_{3} s_{3} \in S$ and thus $w_{1} u_{2} s_{2}=w_{3} v_{2} s_{2} \in S$, that is to say $\left(w_{1} u_{2}-w_{3} v_{2}\right) s_{2}=0$. Hence, unless $A$ is an integral domain, using the second condition of the last proposition, we can find $t \in S$ such that $t\left(w_{1} u_{2}-w_{3} v_{2}\right)=0 \Rightarrow t w_{1} u_{2}=t w_{3} v_{2}$. Hence, changing $w_{1}$ and $w_{3}$ if necessary, we may finally assume that $w_{1} u_{2}=w_{3} v_{2} \Rightarrow w_{1} u_{1} a_{1}=w_{1} u_{2} a_{2}=w_{3} v_{2} a_{2}=w_{3} v_{3} a_{3}$ as wished. We finally define $S^{-1} A$ to be the quotient of $S \times A$ by the above equivalence relation with $\theta: A \rightarrow S^{-1} A: a \rightarrow 1^{-1} a$.

The sum $(s, a)+(t, b)$ will be defined to be $(u s=v t, u a+v b)$ and the product $(s, a) \times(t, b)$ will be defined to be (st, $a b)$.

A similar approach can be used in order to define and construct modules of fractions whenever $S$ satifies the two conditions of the last proposition. For this we need a preliminary lemma:

Lemma 3.1.28: If $S$ is a left Ore set in a $\operatorname{ring} A$ and $M$ is a left module over $A$, the set:

$$
t_{S}(M)=\{x \in M \mid \exists s \in S, s x=0\}
$$

is a submodule of $M$ called the $S$-torsion submodule of $M$.

Proof: If $x, y \in t_{S}(M)$, we may find $s, t \in S$ such that $s x=0, t y=0$. Now, we can find $u, v \in A$ such that $u s=v t \in S$ and we successively get $u s(x+y)=u s x+v t y=0 \Rightarrow x+y \in t_{S}(M)$. Also, $\forall a \in A$, using the Ore condition for $S$, we can find $b \in A, t \in S$ such that $t a=b s$ and we get $\operatorname{tax}=b s x=0 \Rightarrow a x \in t_{S}(M)$.

Q.E.D.

Definition 3.1.29: By a left module of fractions or left localization of $M$ with respect to $S$, we mean a left module $S^{-1} M$ over $S^{-1} A$ both with a homomorphism $\theta=\theta_{S}: M \rightarrow S^{-1} M: x \rightarrow 1^{-1} x$ such that:

1) Each element of $S^{-1} M$ has the form $s^{-1} \theta(x)$ for $s \in S, x \in M$. 
2) $\operatorname{ker}\left(\theta_{S}\right)=t_{S}(M)$

In order to construct $S^{-1} M$, we shall define an equivalence relation on $S \times M$ by saying that $(s, x) \sim(t, y)$ if there exists $u, v \in A$ such that $u s=v t \in S$ and $u x=v y$. Checking that this relation is reflexive, symmetric and transitive can be done as before (exercise) and we define $S^{-1} M$ to be the quotient of $S \times M$ by this equivalence relation.

The main property of localization is expressed by the following theorem:

Theorem 3.1.30: If one has an exact sequence:

$$
M^{\prime} \stackrel{f}{\longrightarrow} M \stackrel{g}{\longrightarrow} M^{\prime \prime}
$$

then one also has the exact sequence:

$$
S^{-1} M^{\prime} \stackrel{S^{-1} f}{\longrightarrow} S^{-1} M \stackrel{S^{-1} g}{\longrightarrow} S^{-1} M^{\prime \prime}
$$

where $S^{-1} f\left(s^{-1} x\right)=s^{-1} f(x)$.

Proof: As $g \circ f=0$, we also have $S^{-1} g \circ S^{-1} f=0$ and thus $i m\left(S^{-1} f\right) \subseteq \operatorname{ker}\left(S^{-1} g\right)$.

In order to prove the reverse inclusion, let $s^{-1} x \in \operatorname{ker}\left(S^{-1} g\right)$. We have therefore $s^{-1} g(x)=0$ in $S^{-1} M^{\prime \prime}$ and there exists $t \in S$ such that $t g(x)=g(t x)=0$ in $M^{\prime \prime}$. As the initial sequence is exact, we can find $x^{\prime} \in M^{\prime}$ such that $t x=f\left(x^{\prime}\right)$. Accordingly, in $S^{-1} M$ we have $s^{-1} x=s^{-1} t^{-1} t x=(t s)^{-1} t x=(t s)^{-1} f\left(x^{\prime}\right)=S^{-1} f\left((t s)^{-1} x^{\prime}\right)$ and thus $\operatorname{ker}\left(S^{-1} g\right) \subseteq i m\left(S^{-1} f\right)$.

Q.E.D.

The proof of the following corollary is left to the reader as an exercise:

Corollary 3.1.31: If $M^{\prime}$ and $M^{\prime \prime}$ are submodules of an $A$-module $M$ and $S$ is a multiplicatively closed subset of $A$, we have the relations:

- $S^{-1}\left(M^{\prime} \cap M^{\prime \prime}\right)=\left(S^{-1} M^{\prime}\right) \cap\left(S^{-1} M^{\prime \prime}\right)$.

- $S^{-1}\left(M^{\prime}+M^{\prime \prime}\right)=\left(S^{-1} M^{\prime}\right)+\left(S^{-1} M^{\prime \prime}\right)$.

- $S^{-1}\left(M^{\prime} \oplus M^{\prime \prime}\right)=\left(S^{-1} M^{\prime}\right) \oplus\left(S^{-1} M^{\prime \prime}\right)$.

- $S^{-1}\left(M / M^{\prime}\right)=\left(S^{-1} M\right) /\left(S^{-1} M^{\prime}\right)$.

We now turn to the definition and brief study of tensor products of modules over rings that will not be necessarily commutative unless stated explicitly.

Let $M=M_{A}$ be a right $A$-module and $N={ }_{A} N$ be a left $A$-module. We may introduce the free $\mathbb{Z}$-module made by finite formal linear combinations of elements of $M \times N$ with coefficients in $\mathbb{Z}$.

Definition 3.1.32: The tensor product of $M$ and $N$ over $A$ is the $\mathbb{Z}$-module $M \otimes_{A} N$ obtained by quotienting the above $\mathbb{Z}$-module by the submodule generated by the elements of the form:

$$
\left(x+x^{\prime}, y\right)-(x, y)-\left(x^{\prime}, y\right),\left(x, y+y^{\prime}\right)-(x, y)-\left(x, y^{\prime}\right),(x a, y)-(x, a y)
$$

and the image of $(x, y)$ will be denoted by $x \otimes y$.

It follows from the definition that we have the relations:

$$
\left(x+x^{\prime}\right) \otimes y=x \otimes y+x^{\prime} \otimes y, x \otimes\left(y+y^{\prime}\right)=x \otimes y+x \otimes y^{\prime}, x a \otimes y=x \otimes a y
$$

and there is a canonical isomorphism $M \otimes_{A} A \simeq M, A \otimes_{A} N \simeq N$. When $A$ is commutative, we may use left modules only and $M \otimes_{A} N$ becomes a left $A$-module.

Example 3.1.33: If $A=\mathbb{Z}, M=\mathbb{Z} / 2 \mathbb{Z}$ and $N=\mathbb{Z} / 3 \mathbb{Z}$, we have $(\mathbb{Z} / 2 \mathbb{Z}) \otimes_{\mathbb{Z}}(\mathbb{Z} / 3 \mathbb{Z})=0$ because $x \otimes y=$ $3(x \otimes y)-2(x \otimes y)=x \otimes 3 y-2 x \otimes y=0-0=0$.

As a link with localization, we let the reader prove that the multiplication map $S^{-1} A \times M \rightarrow S^{-1} M$ given by $\left(s^{-1} a, x\right) \rightarrow s^{-1} a x$ induces an isomorphism $S^{-1} A \otimes_{A} M \rightarrow S^{-1} M$ of modules over $S^{-1} A$ when $S^{-1} A$ is considered 
as a right module over $A$ and $M$ as a left module over $A$.

When $A$ is a commutative integral domain and $S=A-\{0\}$, the field $K=Q(A)=S^{-1} A$ is called the field of fractions of $A$ and we have the short exact sequence:

$$
0 \longrightarrow A \longrightarrow K \longrightarrow K / A \longrightarrow 0
$$

If now $M$ is a left $A$-module, we may tensor this sequence by $M$ on the right with $A \otimes M=M$ but we do not get in general an exact sequence. The defect of exactness on the left is nothing else but the torsion submodule $t(M)=\{m \in M \mid \exists 0 \neq a \in A, a m=0\} \subseteq M$ and we have the long exact sequence:

$$
0 \longrightarrow t(M) \longrightarrow M \longrightarrow K \otimes_{A} M \longrightarrow K / A \otimes_{A} M \longrightarrow 0
$$

as we may describe the central map as follows:

$$
m \longrightarrow 1 \otimes m=\frac{a}{a} \otimes m=\frac{1}{a} \otimes a m \quad, \quad \forall 0 \neq a \in A
$$

Such a result, based on the localization technique, allows to understand why controllability has to do with the so-called "simplification" of the transfer matrix. In particular, a module $M$ is said to be a torsion module if $t(M)=M$ and a torsion-free module if $t(M)=0$.

Definition 3.1.34: A module in $\bmod (A)$ is called a free module if it has a basis, that is a system of generators linearly independent over $A$. When a module $F$ is free, the number of generators in a basis, and thus in any basis (exercise), is called the rank of $F$ over $A$ and is denoted by $\operatorname{rank}_{A}(F)$. In particular, if $F$ is free of finite rank $r$, then $F \simeq A^{r}$.

More generally, if $M$ is any module over a ring $A$ and $F$ is a maximum free submodule of $M$, then $M / F=T$ is a torsion module. Indeed, if $x \in M, x \notin F$, then one can find $a \in A$ such that $a x \in F$ because, otherwise, $F \subset\{F, x\}$ should be free submodules of $M$ with a strict inclusion. In that case, the rank of $M$ is by definition the rank of $F$ over $A$ and one has equivalently:

Lemma 3.1.35: $r k_{A}(M)=\operatorname{dim}_{K}\left(K \otimes_{A} M\right)$.

Proof: Taking the tensor product by $K$ over $A$ of the short exact sequence $0 \rightarrow F \rightarrow M \rightarrow T \rightarrow 0$, we get an isomorphism $K \otimes_{A} F \simeq K \otimes_{A} M$ because $K \otimes_{A} T=0$ (exercise) and the lemma follows from the definition of the rank.

Q.E.D.

We now provide two proofs of the additivity property of the rank, the second one being also valid for noncommutative rings.

Proposition 3.1.36: If $0 \rightarrow M^{\prime} \stackrel{f}{\rightarrow} M \stackrel{g}{\rightarrow} M^{\prime \prime} \rightarrow 0$ is a short exact sequence of modules over a ring $A$, then we have $r k_{A}(M)=r k_{A}\left(M^{\prime}\right)+r k_{A}\left(M^{\prime \prime}\right)$.

Proof 1: Using localization with respect to the multiplicatively closed subset $S=A-\{0\}$, this proposition is just a straight consequence of the definition of rank and the fact that localization preserves exactness.

Proof 2: Let us consider the following diagram with exact left/right columns and central row:

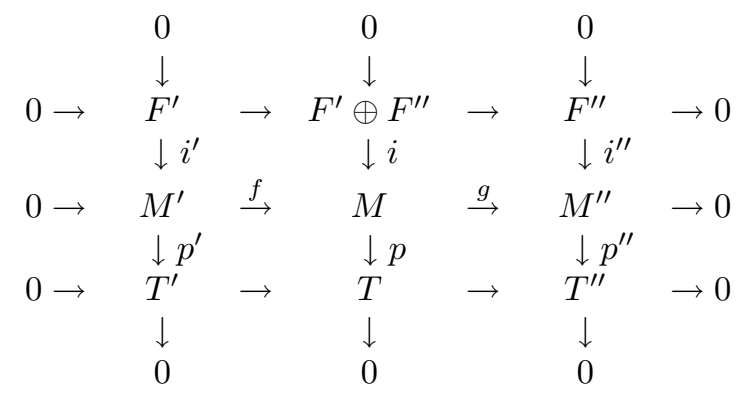

where $F^{\prime}\left(F^{\prime \prime}\right)$ is a maximum free submodule of $M^{\prime}\left(M^{\prime \prime}\right)$ and $T^{\prime}=M^{\prime} / F^{\prime}\left(T^{\prime \prime}=M^{\prime \prime} / F^{\prime \prime}\right)$ is a torsion module. Pulling back by $g$ the image under $i^{\prime \prime}$ of a basis of $F^{\prime \prime}$, we may obtain by linearity a map $\sigma: F^{\prime \prime} \rightarrow M$ and we 
define $i=f \circ i^{\prime} \circ \pi^{\prime}+\sigma \circ \pi^{\prime \prime}$ where $\pi^{\prime}: F^{\prime} \oplus F^{\prime \prime} \rightarrow F^{\prime}$ and $\pi^{\prime \prime}: F^{\prime} \oplus F^{\prime \prime} \rightarrow F^{\prime \prime}$ are the canonical projections on each factor of the direct sum. We have $\left.i\right|_{F^{\prime}}=f \circ i^{\prime}$ and $g \circ i=g \circ \sigma \circ \pi^{\prime \prime}=i^{\prime \prime} \circ \pi^{\prime \prime}$. Hence, the diagram is commutative and thus exact with $r k_{A}\left(F^{\prime} \oplus F^{\prime \prime}\right)=r k_{A}\left(F^{\prime}\right)+r k_{A}\left(F^{\prime \prime}\right)$ trivially. Finally, if $T^{\prime}$ and $T^{\prime \prime}$ are torsion modules, it is easy to check that $T$ is a torsion module too and $F^{\prime} \oplus F^{\prime \prime}$ is thus a maximum free submodule of $M$.

Q.E.D.

Definition 3.1.37: If $f: M \rightarrow N$ is any morphism, the $r a n k$ of $f$ will be defined to be $r k_{A}(f)=r k_{A}(i m(f))$.

We provide a few additional properties of the rank that will be used in the sequel. For this we shall set $M^{*}=\operatorname{hom}_{A}(M, A)$ and, for any moprphism $f: M \rightarrow N$ we shall denote by $f^{*}: N^{*} \rightarrow M^{*}$ the corresponding morphism which is such that $f^{*}(h)=h \circ f, \forall h \in \operatorname{hom}_{A}(N, A)$.

Proposition 3.1.38: When $A$ is a commutative integral domain and $M$ is a finitely presented module over $A$, then $r k_{A}(M)=r k_{A}\left(M^{*}\right)$.

Proof: Applying $\operatorname{hom}_{A}(\bullet, A)$ to the short exact sequence in the proof of the preceding lemma while taking into account $T^{*}=0$, we get a monomorphism $0 \rightarrow M^{*} \rightarrow F^{*}$ and obtain therefore $r k_{A}\left(M^{*}\right) \leq r k_{A}\left(F^{*}\right)$. However, as $F \simeq A^{r}$ with $r<\infty$ because $M$ is finitely generated, we get $F^{*} \simeq A^{r}$ too because $A^{*} \simeq A$. It follows that $r k_{A}\left(M^{*}\right) \leq r k_{A}\left(F^{*}\right)=r k_{A}(F)=r k_{A}(M)$ and thus $r k_{A}\left(M^{*}\right) \leq r k_{A}(M)$.

Now, if $F_{1} \stackrel{d}{\rightarrow} F_{0} \rightarrow M \rightarrow 0$ is a finite presentation of $M$, applying $\operatorname{hom}_{A}(\bullet, A)$ to this presentation, we get the ker/coker exact sequence:

$$
0 \leftarrow N \leftarrow F_{1}^{*} \stackrel{d^{*}}{\leftarrow} F_{0}^{*} \leftarrow M^{*} \leftarrow 0
$$

Applying $\operatorname{hom}_{A}(\bullet, A)$ to this sequence while taking into account the isomorphisms $F_{0}^{* *} \simeq F_{0}, F_{1}^{* *} \simeq F_{1}$, we get the ker/coker exact sequence:

$$
0 \rightarrow N^{*} \rightarrow F_{1} \stackrel{d}{\rightarrow} F_{0} \rightarrow M \rightarrow 0
$$

Counting the ranks, we obtain:

$$
r k_{A}(N)-r k_{A}\left(M^{*}\right)=r k_{A}\left(F_{1}^{*}\right)-r k_{A}\left(F_{0}^{*}\right)=r k_{A}\left(F_{1}\right)-r k_{A}\left(F_{0}\right)=r k_{A}\left(N^{*}\right)-r k_{A}(M)
$$

and thus:

$$
\left(r k_{A}(M)-r k_{A}\left(M^{*}\right)\right)+\left(r k_{A}(N)-r k_{A}\left(N^{*}\right)\right)=0
$$

As both two numbers in this sum are non-negative, they must be zero and we finally get $r k_{A}(M)=r k_{A}\left(M^{*}\right), r k_{A}(N)=$ $r k_{A}\left(N^{*}\right)$.

Q.E.D.

Corollary 3.1.39: Under the condition of the proposition, we have $r k_{A}(f)=r k_{A}\left(f^{*}\right)$.

Proof: Introducing the ker/coker exact sequence:

$$
0 \rightarrow K \rightarrow M \stackrel{f}{\rightarrow} N \rightarrow Q \rightarrow 0
$$

we have: $r k_{A}(f)+r k_{A}(Q)=r k_{A}(N)$. Applying $h_{o m}(\bullet, A)$ and taking into account Theorem 3.1.14, we have the exact sequence:

$$
0 \rightarrow Q^{*} \rightarrow N^{*} \stackrel{f^{*}}{\rightarrow} M^{*}
$$

and thus : $r k_{A}\left(f^{*}\right)+r k_{A}\left(Q^{*}\right)=r k_{A}\left(N^{*}\right)$. Using the preceding proposition, we get $r k_{A}(Q)=r k_{A}\left(Q^{*}\right)$ and $r k_{A}(N)=r k_{A}\left(N^{*}\right)$, that is to say $r k_{A}(f)=r k_{A}\left(f^{*}\right)$.

Q.E.D.

\section{I.3.2 Homological Algebra}

Having in mind the introductory section, we now need a few definittions and results from homological algebra $[4,12,23]$. In all that follows, $A, B, C, \ldots$ are modules over a ring $A$ or vector spaces over a field $k$ and the linear 
maps are making the diagrams commutative.

We start recalling the well known Cramer's rule for linear systems through the exactness of the ker/coker sequence for modules. We introduce the notations $r k=$ rank, nb = number, dim = dimension, ker $=$ kernel, im $=i m a g e$, coker $=$ cokernel. When $\Phi: A \rightarrow B$ is a linear map (homomorphism), we introduce the so-called ker/coker exact sequence:

$$
0 \longrightarrow \operatorname{ker}(\Phi) \longrightarrow A \stackrel{\Phi}{\longrightarrow} B \longrightarrow \operatorname{coker}(\Phi) \longrightarrow 0
$$

where $\operatorname{coker}(\Phi)=B / \operatorname{im}(\Phi)$.

In the case of vector spaces over a field $k$, we successively have $r k(\Phi)=\operatorname{dim}(\operatorname{im}(\Phi)), \operatorname{dim}(\operatorname{ker}(\Phi))=\operatorname{dim}(A)-$ $r k(\Phi), \operatorname{dim}(\operatorname{coker}(\Phi))=\operatorname{dim}(B)-r k(\Phi)=n b$ of compatibility conditions, and obtain by substraction:

$$
\operatorname{dim}(k e r(\Phi))-\operatorname{dim}(A)+\operatorname{dim}(B)-\operatorname{dim}(\operatorname{coker}(\Phi))=0
$$

In the case of modules, using localization, we may replace the dimension by the rank and obtain the same relations because of the additive property of the rank. The following theorem is essential:

Snake Theorem 3.2.1: When one has the following commutative diagram resulting from the the two central vertical short exact sequences by exhibiting the three corresponding horizontal ker/coker exact sequences:

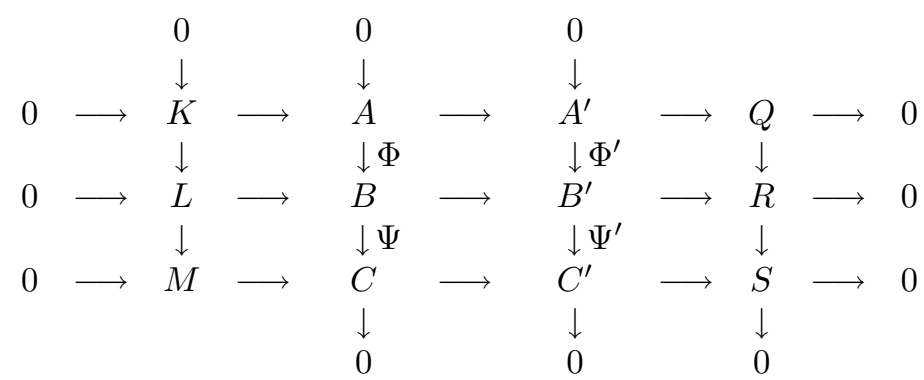

then there exists a connecting map $M \longrightarrow Q$ both with a long exact sequence:

$$
0 \longrightarrow K \longrightarrow L \longrightarrow M \longrightarrow Q \longrightarrow R \longrightarrow S \longrightarrow 0
$$

Proof: We start constructing the connecting map by using the following succession of elements:

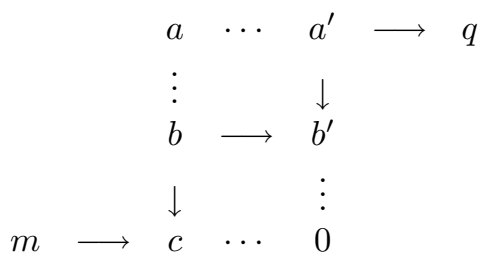

Indeed, starting with $m \in M$, we may identify it with $c \in C$ in the kernel of the next horizontal map. As $\Psi$ is an epimorphism, we may find $b \in B$ such that $c=\Psi(b)$ and apply the next horizontal map to get $b^{\prime} \in B^{\prime}$ in the kernel of $\Psi^{\prime}$ by the commutativity of the lower square. Accordingly, there is a unique $a^{\prime} \in A^{\prime}$ such that $b^{\prime}=\Phi^{\prime}\left(a^{\prime}\right)$ and we may finally project $a^{\prime}$ to $q \in Q$. The map is well defined bacause, if we take another lift for $c$ in $B$, it will differ from $b$ by the image under $\Phi$ of a certain $a \in A$ having zero image in $Q$ by composition. The remaining of the proof is similar and left to the reader as an exercise. The above explicit procedure will not be repeated.

Q.E.D.

We may now introduce cohomology theory through the following definition:

Definition 3.2.2: If one has a sequence $A \stackrel{\Phi}{\longrightarrow} B \stackrel{\Psi}{\longrightarrow} C$, then one may introduce with coboundary $=i m(\Phi) \subseteq$ $\operatorname{ker}(\Psi)=$ cocycle $\subseteq B$ and define the cohomology at $B$ to be the quotient cocycle/coboundary. 
Theorem 3.2.3: The following commutative diagram where the two central vertical sequences are long exact sequences and the horizontal lines are ker/coker exact sequences:

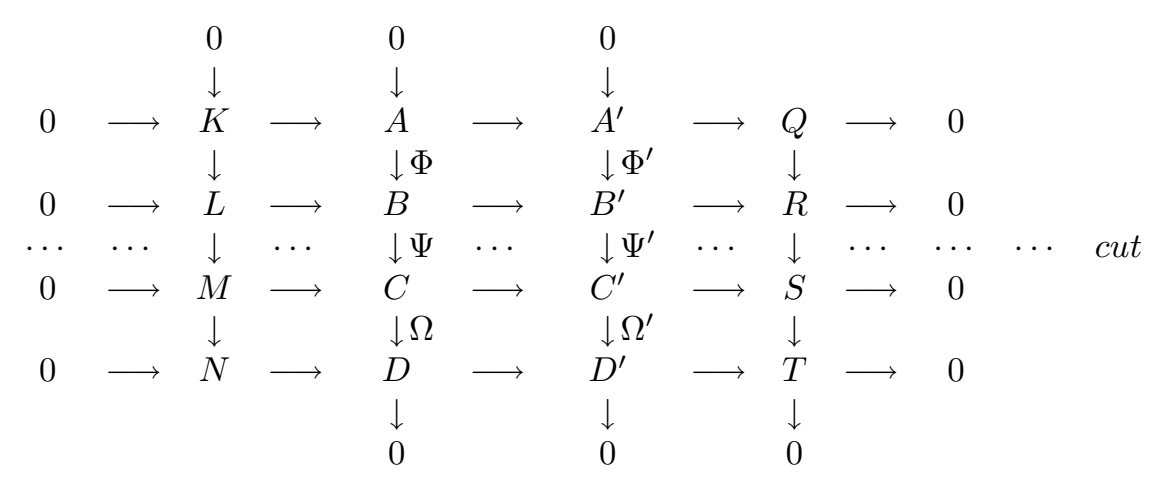

induces an isomorphism between the cohomology at $M$ in the left vertical column and the kernel of the morphism $Q \rightarrow R$ in the right vertical column.

Proof: Let us "cut" the preceding diagram into the following two commutative and exact diagrams by taking into account the relations $\operatorname{im}(\Psi)=\operatorname{ker}(\Omega), \operatorname{im}\left(\Psi^{\prime}\right)=\operatorname{ker}\left(\Omega^{\prime}\right)$ :

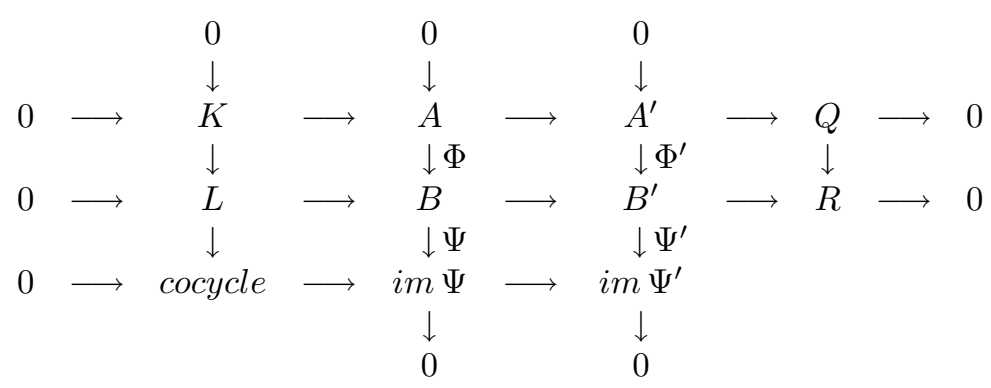

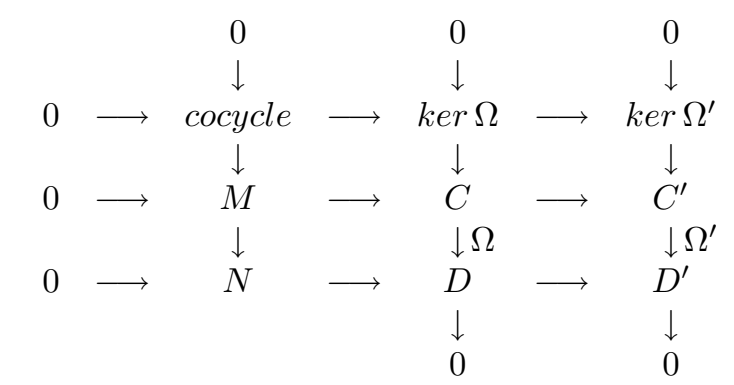

Finally, using the snake theorem, we successively obtain:

$$
\begin{array}{cccc}
\Longrightarrow & \exists & 0 \longrightarrow K \longrightarrow L \stackrel{\Psi}{\longrightarrow} \text { cocycle } \longrightarrow Q \longrightarrow R & \text { exact } \\
\Longrightarrow & \exists & 0 \longrightarrow \text { coboundary } \longrightarrow \text { cocycle } \longrightarrow \operatorname{ker}(Q \longrightarrow R) \longrightarrow 0 & \text { exact } \\
\text { cohomologyat } M \simeq \operatorname{ker}(Q \stackrel{R}{\longrightarrow} & &
\end{array}
$$

Q.E.D.

We now introduce the extension functor in an elementary manner, using the standard notation $h_{0} m_{A}(M, A)=$ $M^{*}$. First of all, by a free resolution of an $A$-module $M$, we understand a long exact sequence:

$$
\ldots \stackrel{d_{2}}{\longrightarrow} F_{1} \stackrel{d_{1}}{\longrightarrow} F_{0} \longrightarrow M \longrightarrow 0
$$

where $F_{0}, F_{1}, \ldots$ are free modules, that is to say modules isomorphic to powers of $A$ and $M=\operatorname{coker}\left(d_{1}\right)=F_{0} /$ im $\left(d_{1}\right)$. We may take out $M$ and obtain the deleted sequence:

$$
\ldots \stackrel{d_{2}}{\longrightarrow} F_{1} \stackrel{d_{1}}{\longrightarrow} F_{0} \longrightarrow 0
$$


which is of course no longer exact. If $N$ is any other $A$-module, we may apply the functor $h_{0} m_{A}(\bullet, N)$ and obtain the sequence:

$$
\ldots \stackrel{d_{2}^{*}}{\longleftarrow} \operatorname{hom}_{A}\left(F_{1}, N\right) \stackrel{d_{1}^{*}}{\longleftarrow} \operatorname{hom}_{A}\left(F_{0}, N\right) \longleftarrow 0
$$

in order to state:

Definition 3.2.4: $\operatorname{ext}_{A}^{0}(M, N)=\operatorname{ker}\left(d_{1}^{*}\right)=\operatorname{hom}_{A}(M, N), \quad \operatorname{ext}_{A}^{i}(M, N)=k e r\left(d_{i+1}^{*}\right) / \operatorname{im}\left(d_{i}^{*}\right), \forall i \geq 1$

One can prove that the extension modules do not depend on the resolution of $M$ chosen and have the following two main properties, the first of which only is classical $[19,23]$.

Proposition 3.2.5: If $0 \rightarrow M^{\prime} \rightarrow M \rightarrow M^{\prime \prime} \rightarrow 0$ is a short exact sequence of $A$-modules, then we have the following connecting long exact sequence:

$$
\begin{aligned}
0 \rightarrow \operatorname{hom}_{A}\left(M^{\prime \prime}, N\right) \rightarrow \operatorname{hom}_{A}(M, N) & \\
& \rightarrow \operatorname{hom}_{A}\left(M^{\prime}, N\right) \rightarrow \operatorname{ext}_{A}^{1}\left(M^{\prime \prime}, N\right) \rightarrow \ldots
\end{aligned}
$$

of extension modules.

We provide two different proofs of the following proposition:

Proposition 3.2.6: $\operatorname{ext}_{A}^{i}(M, A)$ is a torsion module, $\forall i \geq 1$.

Proof 1: Let $F$ be a maximal free submodule of $M$. From the short exact sequence:

$$
0 \longrightarrow F \longrightarrow M \longrightarrow M / F \longrightarrow 0
$$

where $M / F$ is a torsion module, we obtain the long exact sequence:

$$
\begin{aligned}
\ldots \rightarrow \operatorname{ext}_{A}^{i-1}(F, A) & \rightarrow \operatorname{ext}_{A}^{i}(M / F, A) \\
& \rightarrow \operatorname{ext}_{A}^{i}(M, A) \rightarrow \operatorname{ext}_{A}^{i}(F, A) \rightarrow \ldots
\end{aligned}
$$

From the definitions, we obtain $\operatorname{ext}_{A}^{i}(F, A)=0, \forall i \geq 1$ and thus $\operatorname{ext}_{A}^{i}(M, A) \simeq \operatorname{ext}_{A}^{i}(M / F, A), \forall i \geq 2$. Now it is known that the tensor by the field $K$ of any exact sequence is again an exact sequence. Accordingly, we have from the definition:

$$
\begin{aligned}
K \otimes_{A} \operatorname{ext}_{A}^{i}(M / F & , A) \simeq \operatorname{ext}_{A}^{i}(M / F, K) \\
& \simeq \operatorname{ext}_{K}^{i}\left(K \otimes_{A} M / F, K\right)=0, \forall i \geq 1
\end{aligned}
$$

and we finally obtain from the above sequence $K \otimes_{A} \operatorname{ext}_{A}^{i}(M, A)=0 \Rightarrow \operatorname{ext}_{A}^{i}(M, A)$ torsion, $\forall i \geq 1$.

Proof 2: Having in mind that $B_{i}=i m\left(d_{i}^{*}\right)$ and $Z_{i}=\operatorname{ker}\left(d_{i+1}^{*}\right)$, we obtain $r k\left(B_{i}\right)=r k\left(d_{i}^{*}\right)=r k\left(d_{i}\right)$ and $r k\left(Z_{i}\right)=r k\left(F_{i}^{*}\right)-r k\left(d_{i+1}^{*}\right)=r k\left(F_{i}\right)-r k\left(d_{i+1}\right)$. However, we started from a resolution, that is an exact sequence in which $r k\left(d_{i}\right)+r k\left(d_{i+1}\right)=r k\left(F_{i}\right)$. It follows that $r k\left(B_{i}\right)=r k\left(Z_{i}\right)$ and thus $r k\left(H_{i}\right)=r k\left(Z_{i}\right)-r k\left(B_{i}\right)=0$, that is to say $\operatorname{ext}_{A}^{i}(M, A)$ is a torsion module for $i \geq 1, \forall M \in \bmod (A)$.

Q.E.D.

As we have seen in the Motivating Examples, the same module may have many very different presentations. In particular, we have $[5,14]$ :

Schanuel Lemma 3.2.7: If $F_{1}^{\prime} \stackrel{d_{1}^{\prime}}{\longrightarrow} F_{0}^{\prime} \rightarrow M \rightarrow 0$ and $F_{1}^{\prime \prime} \stackrel{d_{1}^{\prime \prime}}{\longrightarrow} F_{0}^{\prime \prime} \rightarrow M \rightarrow 0$ are two presentations of $M$, there exists a presentation $F_{1} \stackrel{d_{1}}{\longrightarrow} F_{0} \rightarrow M \rightarrow 0$ of $M$ projecting onto the preceding ones.

Definition 3.2.8: An $A$-module $P$ is projective if there exists a free module $F$ and another (thus projective) module $Q$ such that $P \oplus Q \simeq F$. Any free module is projective.

Proposition 3.2.9: The short exact sequence:

$$
0 \longrightarrow M^{\prime} \stackrel{f}{\longrightarrow} M \stackrel{g}{\longrightarrow} M^{\prime \prime} \longrightarrow 0
$$


splits whenever $M^{\prime \prime}$ is projective.

Proposition 3.2.10: When $P$ is a projective module and $N$ is any module, we have $\operatorname{ext}_{A}^{i}(P, N)=0, \forall i \geq 1$.

Proposition 3.2.11: When $P$ is a projective module, applying $\operatorname{hom}_{A}(P, \bullet)$ to any short exact sequence gives a short exact sequence.

\section{I.3.3 System Theory}

We recall a few basic facts from jet theory and system theory $[11,18,24]$.

Let $X$ be a manifold of dimension $n$ with local coordinates $x=\left(x^{1}, \ldots, x^{n}\right)$ and $E$ be a vector bundle over $X$ with local coordinates $\left(x^{i}, y^{k}\right)$, where $i=1, \ldots, n$ for the independent variables, $k=1, \ldots, m$ for the dependent variables, and projection $(x, y) \rightarrow x$. A (local) section $\xi: X \rightarrow E: x \rightarrow(x, \xi(x))$ is defined locally by $y^{k}=\xi^{k}(x)$. Under any change of local coordinates $(x, y) \rightarrow(\bar{x}=\varphi(x), \bar{y}=A(x) y)$ the section changes according to $\bar{y}^{l}=\bar{\xi}^{l}(\bar{x})$ in such a way that $\bar{\xi}^{l}(\varphi(x)) \equiv A_{k}^{l}(x) \xi^{k}(x)$ and we may differentiate successively each member in order to obtain, though in a more and more tedious way, the transition rules for the derivatives $\xi^{k}(x), \partial_{i} \xi^{k}(x), \partial_{i j} \xi^{k}(x), \ldots$ up to order $q$. As usual, we shall denote by $J_{q}(E)$ and call q-jet bundle the vector bundle over $X$ with the same transition rules and local jet coordinates $\left(x, y_{q}\right)$ with $y_{q}=\left(y^{k}, y_{i}^{k}, y_{i j}^{k}, \ldots\right)$ or, more generally $y_{\mu}^{k}$ with $1 \leq|\mu| \leq q$ where $\mu=\left(\mu_{1}, \ldots, \mu_{n}\right)$ is a multi-index of length $|\mu|=\mu_{1}+\ldots+\mu_{n}$ and $\mu+1_{i}=\left(\mu_{1}, \ldots, \mu_{i-1}, \mu_{i}+1, \mu_{i+1}, \ldots, \mu_{n}\right)$. The reader must not forget that the above definiions are standard ones in physics or mechanics because of the use of tensors in electromagnetism or elasticity.

Definition 3.3.1: A system of PD equations on $E$ is a vector sub-bundle $R_{q} \subset J_{q}(E)$ locally defined by a constant rank system of linear equations $A_{k}^{\tau \mu}(x) y_{\mu}^{k}=0$.

Substituting the derivatives of a section in place of the corresponding jet coordinates, then differentiating once with respect to $x^{i}$ and substituting the jet coordinates, we get the first prolongation $R_{q+1} \subset J_{q+1}(E)$, defined by the previous equations and by the new equations $A_{k}^{\tau \mu}(x) y_{\mu+1_{i}}^{k}+\partial_{i} A_{k}^{\tau \mu}(x) y_{\mu}^{k}=0$, and, more generally, the $r$-prolongations $R_{q+r} \subset J_{q+r}(E)$ which need not be vector bundles $\left(x y_{x}-y=0 \Longrightarrow x y_{x x}=0\right)$.

Definition 3.3.2: $R_{q}$ is said to be formally integrable if the $R_{q+r}$ are vector bundles and all the generating PD equations of order $q+r$ are obtained by prolonging $R_{q}$ exactly $r$-times only, $\forall r \geq 0$.

The modern way to deal with a linear system of PDE is to use jet coordinates instead of derivatives in order to define a vector subbundle $R_{q} \subset J_{q}(E)$ by a system of local (nondifferential) equations $A_{k}^{\tau \mu}(x) y_{\mu}^{k}=0$ where we have used Einstein summation. The r-prolongation $\rho_{r}\left(R_{q}\right)=J_{r}\left(R_{q}\right) \cap J_{q+r}(E) \subset J_{r}\left(J_{q}(E)\right)$ or simply $R_{q+r}$ will be obtained by substituting derivatives instead of jet coordinates, differentiating $r$ times in the usual way and substituting again jet coordinates. The projections $\pi_{q+r}^{q+r+s}: J_{q+r+s}(E) \longrightarrow J_{q+r}(E)$ induce maps $\pi_{q+r}^{q+r+s}: R_{q+r+s} \longrightarrow R_{q+r}$ which are not in general surjective and we may introduce the families of vector spaces $R_{q+r}^{(s)}=\pi_{q+r}^{q+r+s}\left(R_{q+r+s}\right) \subseteq R_{q+r}$ which may not be vector bundles for any $r, s \geq 0$.

The symbol $g_{q}=R_{q} \cap S_{q} T^{*} \otimes E \subset J_{q}(E)$ of $R_{q}$ is defined by the linear equations:

$$
A_{k}^{\tau \mu}(x) v_{\mu}^{k}=0 \quad|\mu|=q
$$

We let the reader check that the symbol $g_{q+r}$ of $R_{q+r}$ is similarly defined by the linear equations:

$$
A_{k}^{\tau \mu}(x) v_{\mu+\nu}^{k}=0 \quad|\mu|=q,|\nu|=r
$$

Now, to any set $\left(P^{\tau}\right)$ of polynomials of degree $q$ in $k[\chi]$ with $P^{\tau}=A^{\tau \mu} \chi_{\mu}$ and $\chi_{\mu}=\left(\chi_{1}\right)^{\mu_{1}} \ldots\left(\chi_{n}\right)^{\mu_{n}}$ we may associate a trivial vector bundle with fiber dimension one and the linear system $R_{q}$ defined "locally" by the equations:

$$
A^{\tau \mu} y_{\mu}=0 \quad 0 \leq|\mu| \leq q
$$

on the condition to consider it with locally constant coefficients. We notice that such a property insures all the regularity conditions needed for the applications of the formal theory and allows to associate a linear system 
of PD equations in one unknown with constant coefficients to any system of polynomials generating an ideal in $k[\xi]$.

In any case, from now on we shall suppose that the various symbols and projections already defined are vector bundles over $k$, with a slight abuse of language. Apart from the situation coming from pure algebra as we saw, such a case is quite rare in practice and only happens usually in the study of transitive Lie pseudogroups of transformations [18].

If we introduce the cotangent bundle $T^{*}=T^{*}(X)$ with corresponding tensor, exterior and symmetric products respectively denoted by $\otimes, \Lambda, S$, we may define the Spencer map:

$$
\delta: \Lambda^{s} T^{*} \otimes g_{q+r+1} \longrightarrow \Lambda^{s+1} T^{*} \otimes g_{q+r}
$$

by the following local formulas on families of forms:

$$
\left(\delta_{i} v\right)_{\mu}^{k}=v_{\mu+1_{i}}^{k}(\delta v)_{\mu}^{k}=d x^{i} \wedge \delta_{i} v
$$

One has:

$$
((\delta \circ \delta) v)_{\mu}^{k}=d x^{i} \wedge d x^{j} \wedge v_{\mu+1_{i}+1_{j}}^{k} \equiv 0
$$

and thus $\delta \circ \delta=0$. The cohomology at $\Lambda^{s} T^{*} \otimes g_{q+r}$ of the corresponding sequence is denoted by $H_{q+r}^{s}\left(g_{q}\right)$ as it only depends on $g_{q}$ which is said to be $s$-acyclic if $H_{q+r}^{1}=\ldots=H_{q+r}^{s}=0, \forall r \geq 0$, involutive if it is $n$-acyclic and finite type if $g_{q+r}=0$ for $r$ big enough. Contrary to 2-acyclicity, involutivity can be checked by means of a finite algorithm and $g_{q+r}$ becomes involutive for $r$ big enough whenever $g_{q}$ is not involutive.

Indeed, let us define locally:

$$
\left(g_{q}\right)^{i}=\left\{v \in g_{q} \mid \delta_{1} v=0, \ldots, \delta_{i} v=0\right\}
$$

with $\left(g_{q}\right)^{0}=g_{q},\left(g_{q}\right)^{n}=0$ and introduce the local characters:

$$
\alpha_{q}^{i}=\operatorname{dim}\left(g_{q}\right)^{i-1}-\operatorname{dim}\left(g_{q}\right)^{i}
$$

that satisfy the inequalities: $\alpha_{q}^{1} \geq \ldots \geq \alpha_{q}^{n} \geq 0$.

Then $g_{q}$ is involutive if and only if there exists a system of local coordinates, called $\delta$-regular, such that:

$$
\operatorname{dim}\left(g_{q+1}\right)=\alpha_{q}^{1}+2 \alpha_{q}^{2}+\ldots+n \alpha_{q}^{n}
$$

or, equivalently, if we have the following short exact sequences:

$$
0 \longrightarrow\left(g_{q+1}\right)^{i} \longrightarrow\left(g_{q+1}\right)^{i-1} \stackrel{\delta_{i}}{\longrightarrow}\left(g_{q}\right)^{i-1} \longrightarrow 0
$$

The latter definition is the modern version of the multiplicative and nonmultiplicative variables used by Janet that we now recall and illustrate [5].

For this, let us order the $v_{\mu}^{k}$ according to the lexicographic order on $\mu, k$ and let us solve in cascade the various linear equations defining $g_{q}$ with respect to the highest $v_{\mu}^{k}$ each time. Then, let us associate with each such solved equation the multiplicative variables $\left(x^{1}, \ldots, x^{i}\right)$ if the highest $v_{\mu}^{k}$ is of class $i$, that is to say is such that $\mu_{1}=\ldots=\mu_{i-1}=0$ with $\mu_{i} \neq 0$. Of course, this choice will highly depend on the local coordinates that we can always change linearly. One can prove that the following definitionis equivalent to the previous one.

Definition 3.3.3: $g_{q}$ is said to be involutive if its first prolongation with respect to the only multiplicative variables is producing $g_{q+1}$. In that case, the system of coordinates is said to be $\delta$-regular.

Remark 3.3.4: The case of a finite type symbol is the only situation where one can test 2-acyclicity because one can prove easily that the prolongation of a finite type symbol becomes involutive only when the symbol becomes zero $[18,19]$. Also, when $n=2$, we notice that the symbol of the system $y_{11}=0, y_{12}=0$ is involutive though $\left(x^{1}, x^{2}\right)$ is not $\delta$-regular as we may exchange $x^{1}$ and $x^{2}$ to check the preceding definition. 
Example 3.3.5: With $n=3, q=2, m=1$, we provide an example of a symbol which is finite type and 2-acyclic but not involutive. For this, let us consider the symbol $g_{2}$ defined by the 3 equations:

$$
v_{33}-v_{11}=0, \quad v_{23}=0, \quad v_{22}-v_{11}=0
$$

We easily obtain $\operatorname{dim}\left(g_{2}\right)=3$ with only parametric jets $v_{11}, v_{12}, v_{13}$, then $\operatorname{dim}\left(g_{3}\right)=1$ with only parametric jet $v_{111}$ and, surprisingly, $g_{4}=0$. It follows that $g_{2}$ is finite type but cannot be involutive because, if it were so, all the Spencer $\delta$-sequences should be exact, in particular the one finishing at $\Lambda^{3} T^{*} \otimes g_{3}$. Counting the dimensions in this sequence, we should obtain $g_{3}=0$ and a contradiction. A similar comment should prove that $g_{3}$ is of course finite type but not involutive too but we now prove that $g_{3}$ is 2 -acyclic. For this, using the sequence:

$$
0 \rightarrow g_{5} \rightarrow T^{*} \otimes g_{4} \rightarrow \Lambda^{2} T^{*} \otimes g_{3} \rightarrow \Lambda^{3} T^{*} \otimes g_{2} \rightarrow 0
$$

and the fact that $g_{4}=0 \Rightarrow g_{5}=0$, we just need to prove that the last map on the right is an isomorphism. However, the kernel of this map is defined by the 3 equations:

$$
\begin{aligned}
& v_{11,123}=v_{111,23}+v_{112,31}+v_{113,12}=0 \\
& v_{12,123}=v_{121,23}+v_{122,31}+v_{123,12}=0 \\
& v_{13,123}=v_{131,23}+v_{132,31}+v_{133,12}=0
\end{aligned}
$$

that is to say:

$$
v_{111,23}=0, \quad v_{111,31}=0, \quad v_{111,12}=0
$$

Hence, the last map on the right, being a monomorphism between two spaces of the same dimension 3 is an isomorphism and $H_{3}^{2}\left(g_{2}\right)=0$.

The key (absolutely nontrivial) theorem from which all results can be obtained is the following one that can also be extended to nonlinear systems [18,24]:

Theorem 3.3.6: If $g_{q}$ is 2-acyclic, then $\left(R_{q}^{(1)}\right)_{+r}=R_{q+r}^{(1)}, \forall r \geq 0$.

Definition 3.3.7: A system is said to be formally integrable if $R_{q+r}^{(1)}=R_{q+r}, \forall r \geq 0$ and involutive if it is formally integrable with an involutive symbol.

Accordingly, we have the following criterion for formal integrability which is crucial for applications [18,19,24]:

Corollary 3.3.8: If $g_{q}$ is 2-acyclic and $R_{q}^{(1)}=R_{q}$, then $R_{q}$ is formally integrable.

A delicate inductive use of this criterion provides $[18,19]$ :

Corollary 3.3.9: There is a finite algorithm providing two integers $r, s \geq 0$ such that the system $R_{q+r}^{(s)}$ is formally integrable (involutive) with the same solutions as $R_{q}$.

Example 3.3.10: In order to help the reader maturing these new concepts, we illustrate the preceding effective results by showing out, in the case of the Janet motivating example 2.4 , that $r=3, s=2$ with, totally unexpectedly, $g_{5}^{(2)}=0$.

For this, if $\Phi: E \rightarrow F$ is a morphism between vector bundles, we define the $r$-prolongation $\rho_{r}(\Phi): J_{q+r}(E) \rightarrow$ $J_{r}(F)$ by means of the local induction $\Phi: A_{k}^{\tau \mu}(x) y_{\mu}^{k}=u^{\tau} \Rightarrow \rho_{r}(\Phi): A_{k}^{\tau \mu}(x) y_{\mu+\nu}^{k}+\ldots+\partial_{\nu} A_{k}^{\tau \mu}(x) y_{\mu}^{k}$ for $0 \leq|\nu| \leq r$, according to the well known Leibnitz rules of derivations.

Also, if $\Phi: E \rightarrow F$ is a morphism of vector bundles, we may introduce, as in the subsection $1.3 .2, K=$ $\operatorname{ker}(\Phi), Q=\operatorname{coker}(\Phi)=F / i m(\Phi)$ in the following ker-coker long exact sequence of vector budles:

$$
0 \rightarrow K \rightarrow E \rightarrow F \rightarrow Q \rightarrow 0
$$

where one checks the equality $\operatorname{dim}(K)-\operatorname{dim}(E)+\operatorname{dim}(F)-\operatorname{dim}(Q)=0$ because $\operatorname{dim}(E)-\operatorname{dim}(K)=\operatorname{dim}(\operatorname{im}(\Phi))=$ $\operatorname{dim}(F)-\operatorname{dim}(Q)$. 
Finally, introducing $J_{q+r}^{q}(E)=\operatorname{ker}\left(\pi_{q}^{q+r}\right.$, it is easy to check that $J_{q-1}^{q}(E)=S_{q} T^{*} \otimes E$ and we recall the various above results and definitions in the following commutative $\operatorname{diagram} \operatorname{diag}(q, r, s)$ where we have set $F=J_{q}(E) / R_{q}$ :

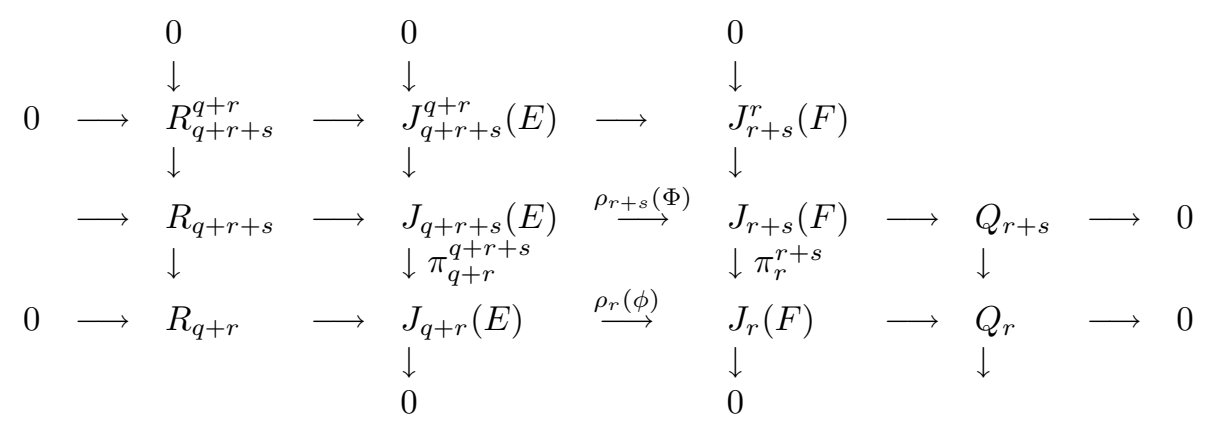

We have $g_{q+r}=R_{q+r}^{q+r-1}$ and the short exact sequences $\operatorname{seq}(q, r, s)$ :

$$
0 \longrightarrow R_{q+r+s}^{q+r} \longrightarrow R_{q+r+s} \longrightarrow R_{q+r}^{(s)} \longrightarrow 0
$$

In the Janet example, $n=3, \operatorname{dim}(E)=m=1, \operatorname{dim}(F)=2, q=2$. We remember that $\operatorname{dim}\left(J_{q}(E)\right)=(n+$ $q) ! / n ! q$ ! and we can easily use computer algebra in order to obtain the numbers $\operatorname{dim}\left(\operatorname{im}\left(\rho_{r}(\Phi)\right)\right)=\operatorname{dim}\left(J_{q+r}(E)\right)-$ $\operatorname{dim}\left(R_{q+r}\right)$ for $r=0,1, \ldots, 6$, that is successively $2,8,20,39,66,102,147$.

We are now ready for applying inductively the preceding theorem and criterion of formal integrability to $R_{2}$ until we reach $R_{5}^{(2)}$ and prove that it is formally integrable with involutive (in fact zero !) symbol or simply involutive. Let us consider $R_{2}$ with $\operatorname{dim}\left(R_{2}\right)=10-2=8$. Then $\operatorname{seq}(2,0,1)$ gives: $\operatorname{dim}\left(R_{2}^{(1)}\right)=\operatorname{dim}\left(R_{3}\right)-\operatorname{dim}\left(g_{3}\right)=$ $12-(10-6)=8=\operatorname{dim}\left(R_{2}\right)$ and thus $R_{2}^{(1)}=R_{2}$. However, we have for $g_{2}$ :

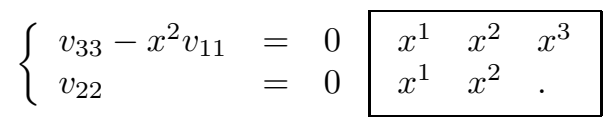

and $g_{2}$ is not involutive because we have for $g_{3}$ :

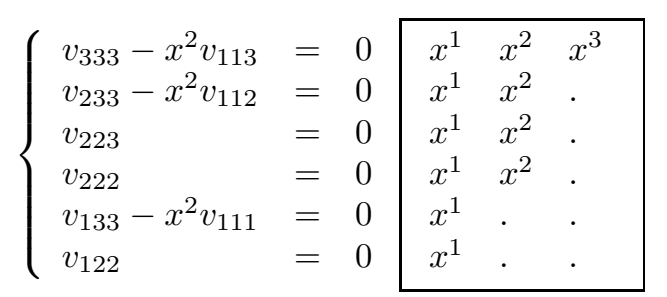

and $\operatorname{dim}\left(g_{3}\right)=10-6=4$ instead of $10-5=5$. Hence $R_{2}$ is not formally integrable. Indeed, $R_{3}^{(1)} \subset R_{3}$ because $\operatorname{seq}(2,1,1)$ gives: $\operatorname{dim}\left(R_{3}^{(1)}\right)=\operatorname{dim}\left(R_{4}\right)-\operatorname{dim}\left(g_{4}\right)=(35-20)-(15-11)=15-4=11<12$ and we may start afresh with $R_{3}^{(1)}$.

Now we notice that $g_{3}$ is involutive because $\operatorname{dim}\left(g_{4}\right)=15-11=4=15-n b$ of multiplicative variables for $g_{3}$. We may thus apply the prolongation theorem to $R_{3}$ and get $\left(R_{3}^{(1)}\right)_{+r}=r_{3+r}^{(1)}$. In particular, if we want to apply the formal integrability criterion to $R_{3}^{(1)}$, we must study $g_{3}^{(1)}$ :

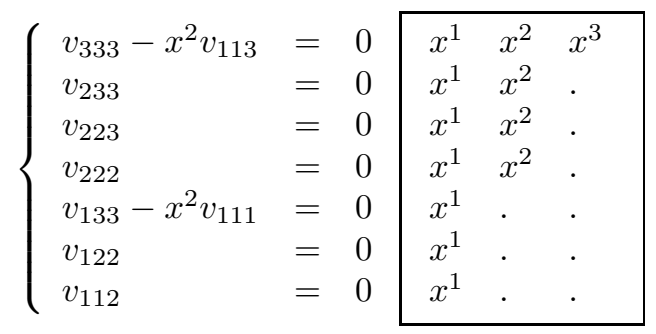

$g_{3}^{(1)}$ is not involutive because of the non-multiplicative variable $x^{3}$ for $v_{112}=0$. However, its first prolongation $g_{4}^{(1)}$ is involutive (exercise). Hence, if we want to check the criterion, we have:

$$
\pi_{4}^{5}\left(\left(R_{4}^{(1)}\right)_{+1}\right)=\pi_{4}^{5}\left(\left(R_{3}^{(1)}\right)_{+2}\right)=\pi_{4}^{5}\left(R_{5}^{(1)}\right)=R_{4}^{(2)} \subseteq R_{4}^{(1)}
$$




$$
\operatorname{dim}\left(R_{4}^{(1)}\right)=\operatorname{dim}\left(R_{5}\right)-\operatorname{dim}\left(g_{5}\right)=(56-39)-4=17-4=13
$$

Now the reader may check by himself that $R_{4}^{(2)}$ is obtained from $R_{4}^{(1)}$ by adding the equation $y_{1111}=0$ and thus $\operatorname{dim}\left(R_{4}^{(2)}\right)=\operatorname{dim}\left(R_{4}^{(1)}\right)-1=12$.

We may start afresh with $R_{4}^{(2)}$ with symbol $g_{4}^{(2)}$ given by:

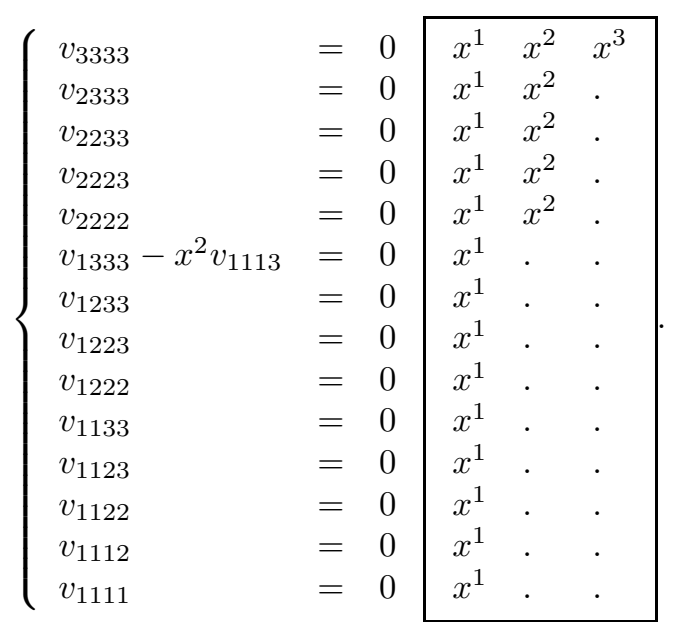

We notice that $g_{4}^{(2)}$ is not involutive because of the non-multiplicative variable $x^{3}$ for $v_{1111}=0$. Its first prolongation is zero and thus trivially involutive. However, we have:

$$
\left(R_{4}^{(2)}\right)_{+1}=\left(\left(R_{4}^{(1)}\right)^{(1)}\right)_{+1}=\left(\left(R_{4}^{(1)}\right)_{+1}\right)^{(1)}=\left(R_{5}^{(1)}\right)^{(1)}=R_{5}^{(2)}
$$

because $g_{4}^{(1)}$ and $g_{3}$ are involutive. But we have $\pi_{4}^{5}\left(R_{5}^{(2)}\right)=R_{4}^{(3)}$ and we deduce from $\operatorname{diag}(2,3,2)$ :

$$
\operatorname{dim}\left(R_{5}^{(2)}\right)=\operatorname{dim}\left(R_{7}\right)-\operatorname{dim}\left(R_{7}^{5}\right)=(120-102)-(64-58)=18-6=12
$$

while we deduce from $\operatorname{diag}(2,2,3)$ :

$$
\operatorname{dim}\left(R_{4}^{(3)}\right)=\operatorname{dim}\left(R_{7}\right)-\operatorname{dim}\left(R_{7}^{4}\right)=(120-102)-(85-79)=18-6=12 .
$$

It follows that $\operatorname{dim}\left(g_{5}^{(2)}\right)=\operatorname{dim}\left(R_{5}^{(2)}\right)-\operatorname{dim}\left(R_{4}^{(3)}\right)=12-12=0$ and $g_{5}^{(2)}=0$ is trivially involutive. However, we have:

$$
\left(R_{5}^{(2)}\right)_{+1}=\left(R_{4}^{(2)}\right)_{+2}=\left(\left(R_{4}^{(1)}\right)^{(1)}\right)_{+2}=\left(\left(R_{4}^{(1)}\right)_{+2}\right)^{(1)}=\left(R_{6}^{(1)}\right)^{(1)}=R_{6}^{(2)}
$$

and $\pi_{5}^{6}\left(R_{6}^{(2)}\right)=R_{5}^{(3)}$. Using similarly $\operatorname{diag}(2,3,3)$, we get:

$$
\operatorname{dim}\left(R_{5}^{(3)}\right)=\operatorname{dim}\left(R_{8}\right)-\operatorname{dim}\left(R_{8}^{5}\right)=(165-147)-(109-103)=18-6=12
$$

and it follows that $R_{5}^{(2)}$ is involutive with zero symbol. In fact, using $\operatorname{diag}(2,4,2)$, we get:

$$
\operatorname{dim}\left(R_{6}^{(2)}\right)=\operatorname{dim}\left(R_{8}\right)-\operatorname{dim}\left(R_{8}^{6}\right)=(165-147)-(81-75)=18-6=12
$$

and we check that $R_{6}^{(2)} \simeq R_{5}^{(2)}$ while $R_{5}^{(3)}=R_{5}^{(2)}$.

We have thus proved that the space of solutions of $R_{2}$ is a 12-dimensional vector space over the constants as it coincides with the space of solutions of the involutive system $R_{5}^{(2)}$ which has zero symbol.

We finally use this result in order to look for the generating compatibility conditions. Indeed, introducing $u$ and $v$ as second members of $R_{2}$, we may therefore add to $R_{5}^{(2)}$ second members involving the derivatives of $u$ and $v$ up to order $7-2=5$. Now, as $R_{5}^{(2)}$ is involutive, the compatibility conditions for the second members only become first order because the criterion only involves one prolongation. Accordingly, the compatibility conditions for $R_{2}$ only involves the derivatives of $u$ and $v$ up to order $1+5=6$ and we have successively:

$$
Q_{1}=Q_{2}=0, \operatorname{dim}\left(Q_{3}\right)=1, \ldots, \operatorname{dim}\left(Q_{6}\right)=21=\operatorname{dim}\left(J_{3}\left(Q_{3}\right)+1\right.
$$


We now specify the correspondence:

$$
S Y S T E M \Leftrightarrow O P E R A T O R \Leftrightarrow M O D U L E
$$

in order to show later on that certain concepts, which are clear in one framework, may become quite obscure in the others and conversely (check this for the formal integrability and torsion concepts for example!).

Having a system of order $q$, say $R_{q} \subset J_{q}(E)$, we can introduce the canonical projection $\Phi: J_{q}(E) \longrightarrow$ $J_{q}(E) / R_{q}=F$ and define a linear differential operator $\mathcal{D}: E \longrightarrow F: \xi(x) \longrightarrow \eta^{\tau}(x)=A_{k}^{\tau \mu}(x) \partial_{\mu} \xi^{k}(x)$. When $\mathcal{D}$ is given, the compatibility conditions for solving $\mathcal{D} \xi=\eta$ can be described in operator form by $\mathcal{D}_{1} \eta=0$ and so on. In general (see the preceding examples), if a system is not formally integrable, it is possible to obtain a formally integrable system, having the same solutions, by "saturating" conveniently the given PD equations through the adjunction of new $\mathrm{PD}$ equations obtained by various prolongations and such a procedure must absolutely be done before looking for the compatibility conditions.

Starting with the work of M. Janet in 1920, effective tests have been provided for checking formal integrability and computer algebra packages dealing with Gröbner bases can be used for such a purpose [4]. However, for reasons that will become clear later on, formal integrability is not sufficient for having certain canonical forms of systems and tests. We have already introduced the Spencer cohomology of the symbol as an intrinsic/coordinate free homological tool for the previous test and now, passing from the symbol to the system, we shall provide an equivalent local description, more useful in practice. For this, changing linearly the independent variables if necessary, we shall solve the maximum number $\beta$ of equations, called equations of class $n$, with respect to the jets of order $q$ and class $n$. Then, we shall solve the maximum number of remaining equations, called equations of class $n-1$, with respect to the jets of order $q$ and class $n-1$, and so on till we get equations of class 1 , the remaining equations being of order $\leq q-1$. For each equation of class $i$ we shall call the variables $x^{1}, \ldots, x^{i}$ (respectively $\left.x^{i+1}, \ldots, x^{n}\right)$ multiplicative (non-multiplicative) while all variables will be non-multiplicative for the equations of order $\leq q-1$. The following definition is essential and studies are in progress in order to test it through computer algebra packages:

Definition 3.3.11: A system of PD equations is said to be involutive if its first prolongation can be achieved by prolonging its equations only with respect to the corresponding multiplicative variables.

It can be proved that this definition is intrinsic though it must be checked in a particular system of coordinates and this point has not been yet overcome by symbolic computations. An involutive system is formally integrable. Also, one can prove that, in this case, the maximum number $\alpha$ of dependent variables that can be given arbitrarily (that are differentially independent) is equal to $m-\beta$. Homogeneous systems with constant coefficients are automatically formally integrable. One can prove $[18,19]$ :

Proposition 3.3.12: When $R_{q}$ is involutive, we shall say that $\mathcal{D}$ is involutive and, in this case only, we get a finite chain of first order involutive operators $\mathcal{D}_{1}, \ldots, \mathcal{D}_{n}$ making up a canonical differential sequence called Janet sequence.

Proof: Let us define the vector bundle $F_{1}$ by the $k e r /$ coker exact sequence of vector budles over $X$ :

$$
0 \longrightarrow g_{q+1} \longrightarrow S_{q+1} T^{*} \otimes E \stackrel{\sigma_{1}(\Phi)}{\longrightarrow} T^{*} \otimes F_{0} \longrightarrow F_{1} \longrightarrow 0
$$

where $\sigma_{1}(\Phi)$ is the restriction of the first prolongation $\rho_{1}(\Phi): J_{q+1}(E) \rightarrow J_{1}\left(F_{0}\right)$ of the epimorphism $\Phi: J_{q}(E) \rightarrow$ $F_{0}$. As $g_{q}$ is involutive and thus at least 2-acyclic, it follows from Theorem 3.2.3 by induction on $r \geq 1$ that we have the following commutative and exact diagram:

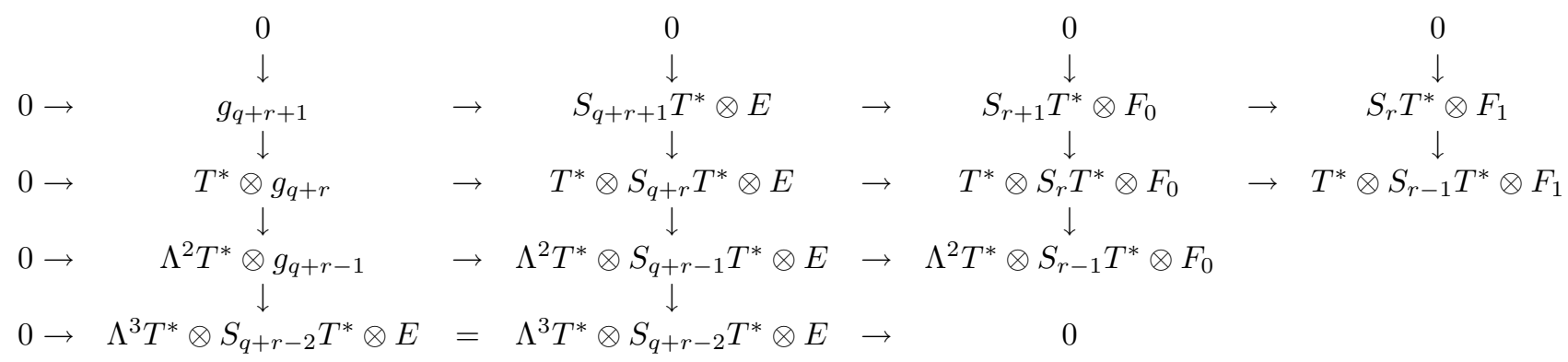


where the vertical sequences are $\delta$-sequences.

Using the exactness of the top rows of the preceding diagrams and the assumption of formal integrability, it now follows by induction on $r \geq 0$ that we have the following commutative and exact diagram:

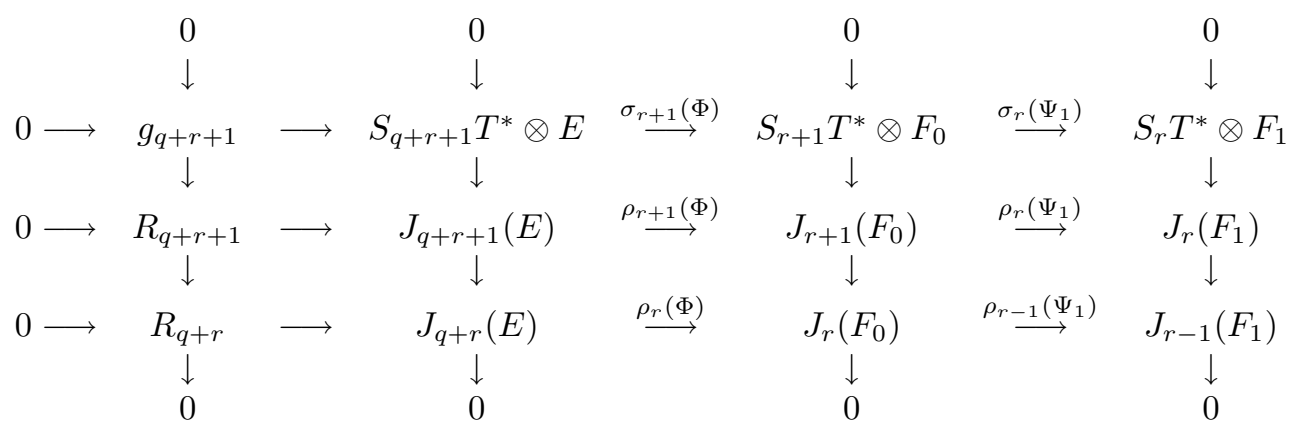

Accordingly, the compatibility conditions of order $r+1$ are nothing else than the $r$-prolongation $\rho_{r}\left(\Psi_{1}\right)$ of the compatibility conditions of order 1 , namely $\Psi_{1}: J_{1}\left(F_{0}\right) \rightarrow F_{1}$ and we may introduce the first order operator $\mathcal{D}_{1}=\Psi_{1} \circ j_{1}$. We let the reader check as an exercise that $\mathcal{D}_{1}$ is again involutive and we may successively construct similarly the first order involutive operators $\mathcal{D}_{2}, \ldots, \mathcal{D}_{n}$.

Finally, cutting the first of the preceding diagrams as we did in the proof of Theorem 3.2 .3 and setting $h_{1}=$ $i m\left(\sigma_{1}(\Phi)\right) \subset T^{*} \otimes F_{0}$, we obtain (exercise) the crucial canonical formula $F_{r}=\Lambda^{r} T^{*} \otimes F_{0} / \delta\left(\Lambda^{r-1} T^{*} \otimes h_{1}\right)$ showing that $\mathcal{D}_{n}$ is always formally surjective.

Q.E.D.

Example 3.3.13: With $n=4, m=1, q=1$, the system $R_{1}$ defined by the two PD equations $\Phi^{2} \equiv y_{4}-x^{3} y_{2}-y=$ $0, \Phi^{3} \equiv y_{3}-x^{4} y_{1}=0$ is not formally integrable as one can easily check $\Phi^{1} \equiv d_{4} \Phi^{2}-d_{3} \Phi^{3}-x^{3} d_{2} \Phi^{2}+x^{4} d_{1} \Phi^{3}-\Phi^{2} \equiv$ $y_{2}-y_{1}=0$. However, the system $R_{1}^{\prime} \subset R_{1}$ defined by the three PD equations $\Phi^{1}=0, \Phi^{2}=0, \Phi^{3}=0$ is involutive with 1 equation of class 4,1 equation of class 3,1 equation of class 2 and one checks the 3 first order compatibility conditions:

$$
\begin{gathered}
\Psi^{3} \equiv d_{4} \Phi^{2}-d_{3} \Phi^{3}-x^{3} d_{2} \Phi^{2}+x^{4} d_{1} \Phi^{3}-\Phi^{2}-\Phi^{1}=0 \\
\Psi^{2} \equiv d_{4} \Phi^{1}-d_{2} \Phi^{3}+d_{1} \Phi^{3}-x^{3} \Phi^{1}-\Phi^{1}=0 \\
\Psi^{1} \equiv d_{3} \Phi^{1}-d_{2} \Phi^{2}+d_{1} \Phi^{2}-x^{4} d_{1} \Phi^{1}=0
\end{gathered}
$$

This is again an involutive system with 2 equations of class 4,1 equation of class 3 and 1 single compatibility condition of class 4, namely:

$$
\left(d_{4}-x^{3} d_{2}-1\right) \Psi^{1}+\left(x^{4} d_{1}-d_{3}\right) \Psi^{2}+\left(d_{2}-d_{1}\right) \Psi^{3}=0
$$

ending the construction of the Janet sequence.

Example 3.3.14: With $n=3, m=1, q=2$, let us consider the homogeneous second order system $\Phi^{1} \equiv$ $y_{22}-y_{11}=0, \Phi^{2} \equiv y_{23}=0, \Phi^{3} \equiv y_{33}-y_{11}=0$. This system is formally integrable though one needs 2 prolongations to get involution (all jets of order 4 are nul !). There are 3 compatibility conditions of order two, namely $d_{33} \Phi^{2}-d_{23} \Phi^{3}-d_{11} \Phi^{2}=0, d_{33} \Phi^{1}-d_{22} \Phi^{3}+d_{11} \Phi^{3}-d_{11} \Phi^{1}=0, d_{23} \Phi^{1}-d_{22} \Phi^{2}+d_{11} \Phi^{2}=0$ and this homogeneous system is again formally integrable though not involutive.

We shall now prove that the "Kalman form" is nothing else but a particular case of the so-called "Spencer form" existing in the formal theory of systems of PD equations, as no reference to control theory is needed.

For simplicity and in a sketchy way, we may say that the Spencer method amounts to use the canonical inclusion $J_{q+1} \subset J_{1}\left(J_{q}(E)\right)$ through the identification $y_{\mu, i}^{k}=y_{\mu+1_{i}}^{k}$ in order to induce a canonical inclusion $R_{q+1} \subset J_{1}\left(R_{q}\right)$ allowing to consider $R_{q+1}$ as a first order system on $R_{q}$, whenever $R_{q+1}$ is a vector bundle. When $R_{q}$ is involutive, this is the Spencer form and the corresponding canonical Janet sequence is called second Spencer sequence $[18,19,24]$. It is not so well known that such a method, which allows to bring a system of order $q$ to a system of order 1 , is only truly useful when $R_{q}$ is formally integrable. Indeed, in this case and only in this case, $R_{q+1}$ can be considered as a first order system over $R_{q}$, without equations of order zero. The following example clarifies this 
delicate point:

Example 3.3.15: Looking back to the preceding example, we notice that $R_{2} \longrightarrow R_{1}$ is not surjective and, introducing the parametric jets (derivatives) $z^{1}=y, z^{2}=y_{1}, z^{3}=y_{2}$ for $R_{1}$, then $R_{2} \subset J_{1}\left(R_{1}\right)$ is defined by first order PD equations and by the zero order equation $z^{3}-z^{2}=0$. On the contrary, if we introduce the new formally integrable system $R_{1}^{\prime}=R_{1}^{(1)} \subset R_{1}$, projection of $R_{2}$ in $R_{1}$, the parametric jets of $R_{1}^{\prime}$ are now only $z^{1}=y, z^{2}=y_{1}$. Though this is not evident at first sight, they are linked by the 7 first order PD equations:

$$
z_{4}^{1}-x^{3} z^{2}-z^{1}=0, z_{4}^{2}-x^{3} z_{1}^{2}-z^{2}=0, z_{3}^{1}-x^{4} z^{2}=0, z_{3}^{2}-x^{4} z_{1}^{2}=0, z_{2}^{1}-z^{2}=0, z_{2}^{2}-z_{1}^{2}=0, z_{1}^{1}-z^{2}=0
$$

because $\operatorname{dim}\left(R_{2}^{\prime}\right)=10-9=4 \times 2-7=1$.

According to the preceding comments and with no loss of generality, we consider from now on a first order system $R_{1} \subset J_{1}(E)$ over $E$ with $R_{1} \longrightarrow E$ surjective and we shall bring such a system to a canonical form, noticing that similar methods can be used for nonlinear systems too as in [19]. For this, changing linearly the system of coordinates if necessary, we may manage to have a maximum number of PD equations solved with respect to the jets $y_{n}^{1}, \ldots, y_{n}^{\beta_{1}^{n}}$, called equations of class $n$. The remaining equations only contain jets with respect to $x^{1}, \ldots, x^{n-1}$ and, changing again linearly the system of coordinates if necessary, we may manage to have a maximum number of PD equations solved with respect to $y_{n-1}^{1}, \ldots, y_{n-1}^{\beta_{1}^{n-1}}$ with $\beta_{1}^{n-1} \leq \beta_{1}^{n}$, called equations of class $n-1$ and so on, till we get the equations of class 1 if any. The complements to $m$ of the $\beta$-integers are the characters (exercise).

Definition 3.3.16: A first order system is said to be involutive if it can be brought to such a canonical form and all the equations of second order can be obtained by differentiating/prolonging the equations of class $i$ with respect to $x^{1}, \ldots, x^{i}$ only, for $i=1, \ldots, n$.

In this situation, $x^{1}, \ldots, x^{i}$ are called multiplicative variables while $x^{i+1}, \ldots, x^{n}$ are called non-multiplicative variable for the equations of class $i$.

Example 3.3.17: The system $R_{1}^{\prime}$ defined in the preceding example is easily seen to be in canonical form and involutive with characters $(1,1,1,0)$. In particular, the equation $y_{2}-y_{1}=0$ is of class 2 with multiplicative variables $x^{1}, x^{2}$ and non-multiplicative variables $x^{3}, x^{4}$. One has $d_{3}\left(y_{2}-y_{1}\right) \equiv d_{2}\left(y_{3}-x^{4} y_{1}\right)-d_{1}\left(y_{3}-x^{4} y_{1}\right)+x^{4} d_{1}\left(y_{2}-y_{1}\right)$

Denoting by $z$ the variables $y^{\beta_{1}^{n+1}}, \ldots, y^{m}$ and using elementary properties of the characteristic variety to be found in section 6, one can prove that the $z$ can be chosen arbitrarily (differential transcendence basis)[ ]. More generaly, if $R_{q}$ is formally integrable with an involutive symbol, that is to say a symbol such that all the groups of Spencer $\delta$-cohomology vanish, the corresponding first order system $R_{q+1} \subset J_{1}\left(R_{q}\right)$ is involutive in the above sense.

It now remains to introduce the linear combinations $\bar{y}^{k}=y^{k}-\operatorname{term}(z)$ for $k=1, \ldots, \beta_{1}^{n}$ that will allow to "absorb" the $z_{n}$ in the $y_{n}$. The generalization of the Kalman form finally depends on the following striking though technical result on involutive systems that does not seem to have been previously known:

Proposition 3.3.18: The new equations of class $n$ only contain $z, z_{1}, \ldots, z_{n-1}$ while the equations of class $1, \ldots, n-1$ no more contain $z$ or its jets.

Proof: $x^{n}$ is a non-multiplicative variable for the equations of class $1, \ldots, n-1$. Hence, if $z$ or any jet of order one should appear in one of the latter equations, by prolonging this equation with respect to $x^{n}$ and using the involutiveness property, one should get a linear combination of equations of various classes, prolonged with respect to $x^{1}, \ldots, x^{n-1}$ and this is not possible as only $z, z_{1}, \ldots, z_{n-1}$ do appear.

Q.E.D.

Example 3.3.19: With $n=2, m=3, q=1$, let us consider the following linear involutive first order system in solved form:

$$
\Phi^{3} \equiv y_{2}^{2}-y_{1}^{2}+z_{2}-z_{1}-z=0, \Phi^{2} \equiv y_{2}^{1}-y_{1}^{2}-z_{2}-z_{1}-z=0, \Phi^{1} \equiv y_{1}^{1}-y_{1}^{2}-2 z_{1}=0
$$

In this system, $\Phi^{2}$ and $\Phi^{3}$ are of class 2 while $\Phi^{1}$ is of class 1 . Setting $\bar{y}^{1}=y^{1}-z, \bar{y}^{2}=y^{2}+z$, we get the new system: 


$$
\Phi^{3} \equiv \bar{y}_{2}^{2}-\bar{y}_{1}^{2}-z=0, \Phi^{2} \equiv \bar{y}_{2}^{1}-\bar{y}_{1}^{2}-z=0, \Phi^{1} \equiv \bar{y}_{1}^{1}-\bar{y}_{1}^{2}=0
$$

and $z$ or its jets no longer appear in $\Phi^{1}$.

In order to study differential modules, for simplicity we shall forget about changes of coordinates and consider trivial bundles. Let $K$ be a differential field with $n$ commuting derivations $\partial_{1}, \ldots, \partial_{n}$, that is to say $K$ is a field and $\partial_{i}: K \rightarrow K$ satisfies $\partial_{i}(a+b)=\partial_{i} a+\partial_{i} b, \partial_{i}(a b)=\left(\partial_{i} a\right) b+a \partial_{i} b, \forall a, b \in K, \forall i=1, \ldots, n$ (say $\mathbb{Q}, \mathbb{Q}\left(x^{1}, \ldots, x^{n}\right)$ or $\mathbb{Q}(a)$ in the previous examples). If $d_{1}, \ldots, d_{n}$ are formal derivatives (pure symbols in computer algebra packages !) which are only supposed to satisfy $d_{i} a=a d_{i}+\partial_{i} a$ in the operator sense for any $a \in K$, we may consider the (non-commutative) ring $D=K\left[d_{1}, \ldots, d_{n}\right]$ of differential operators with coefficients in $K$. If now $y=\left(y^{1}, \ldots, y^{m}\right)$ is a set of differential indeterminates, we let $D$ act formally on $y$ by setting $d_{\mu} y^{k}=y_{\mu}^{k}$ and set $D y=D y^{1}+\ldots+D y^{m}$. Denoting simply by $D \mathcal{D} y$ the subdifferential module generated by all the given OD or PD equations and all their formal derivatives, we may finally introduce the $D$-module $M=D y / D \mathcal{D} y$.

Example 3.3.20: In the Motivating Examples 1 with $K=\mathbb{Q}\left(x^{1}, x^{2}\right)$ and 2 with $K=\mathbb{Q}$, we get $M=0$, while in the Motivating Example 3 with $K=\mathbb{Q}\left(x^{1}, x^{2}, x^{3}\right)$, we get $\operatorname{dim}_{K}(M)=12$.

More generally, with similar definitions, if $A$ is a differential ring, we can consider $D=A[d]$ to be the ring of differential operators with coefficients in $A$ and $D$ is noetherian (is an integral domain) whenever $A$ is noetherian (is an integral domain).

It just remains to prove that even in this non-commutative case, one can also define localization and the torsion submodule. We achieve this in the previous situation of differential operators over a differential field $K$ by means of the following proposition:

Proposition 3.3.21: $D=K[d]$ is an Ore ring, that is to say, given any nonzero $P, Q \in D$, then one can find $A, B \in D$ such that $A P=B Q$.

proof: Let us consider the system with second members $P y=u, Q y=v$ and its prolongations as a linear system over $K$ for the jets of $y, u, v$. If $\operatorname{deg}(P)=p, \operatorname{deg}(Q)=q$, we may suppose that $p \leq q$ and, if we prolong $r$ times, the number of jets of $y$ of order $q+r$ is equal to $(n+q+r) ! / n !(q+r) !=(q+r+1) \ldots(q+r+n) / n !=\frac{1}{n !} r^{n}+\ldots$. Meanwhile, the number of jets of order $r$ of $u$ and $v$ is equal to $2(n+r) ! / n ! r !=\frac{2}{n !} r^{n}+\ldots$. Hence, when $r$ is large enough, the second number is greater than the first and we can eliminate the jets of $y$ by using Cramer's rule over $K$. Accordingly, one can find at least one compatibility condition of the form $A u-B v=0$ and thus $A P=B Q$.

Q.E.D.

The application of the preceding results on localization to $D$ and $S=D-\{0\}$ is immediate and we refer the reader to [19] for more general situations.

\section{I.4 PROBLEM FORMULATION}

Though it seems that we are very far from any possible application, let us now present three problems which, both with the previous examples, look like unrelated with what we already said and between themselves.

Problem 4.1: Let a rigid bar of length $L$ be able to slide horizontally and attach at the end of abcissa $x$ (resp. $x+L$ ) a pendulum of length $l_{1}$ (resp. $l_{2}$ ) with mass $m_{1}$ (resp. $m_{2}$ ), making an angle $\theta_{1}$ (resp. $\theta_{2}$ ) with the downwards vertical axis. Projecting the dynamical equations on the perpendicular to each pendulum in order to eliminate the respective tension, we get:

$$
m_{1}\left(\ddot{x} \cos \theta_{1}+l_{1} \ddot{\theta_{1}}\right)+m_{1} g \sin \theta_{1}=0
$$

where $g$ is the gravity. When $\theta_{1}$ and $\theta_{2}$ are small, we get the following two OD equations that only depend on $l_{1}$ and $l_{2}$ but no longer on $m_{1}$ and $m_{2}$ :

$$
\left\{\begin{array}{l}
\ddot{x}+l_{1} \ddot{\theta}_{1}+g \theta_{1}=0 \\
\ddot{x}+l_{2} \ddot{\theta}_{2}+g \theta_{2}=0
\end{array}\right.
$$

Now it is easy to check experimentally that, when $l_{1} \neq l_{2}$, it is possible to bring any small amplitude motion $\theta_{1}=\theta_{1}(t), \theta_{2}=\theta_{2}(t)$ of the two pendula back to equilibrium $\theta_{1}=0, \theta_{2}=0$, just by choosing a convenient $x=x(t)$ 
and the system is said to be controllable. On the contrary, if $l_{1}=l_{2}$ and unless $\theta_{1}(t)=\theta_{2}(t)$, then it is impossible to bring the pendula back to equilibrium and the system is said to be uncontrollable. A similar question can be asked when $l_{1}=l_{1}(t), l_{2}=l_{2}(t)$ are given, the variation of length being produced by two small engines hidden in the bar.

Hence, a much more general question concerns the controllability of control systems defined by systems of OD or $\mathrm{PD}$ equations as well, like in gasdynamic or magnetohydrodynamic.

In our case, setting $x_{1}=x+l_{1} \theta_{1}, x_{2}=x+l_{2} \theta_{2}$, we get:

$$
\left\{\begin{array}{l}
\ddot{x}_{1}+\left(g / l_{1}\right) x_{1}-\left(g / l_{1}\right) x=0 \\
\ddot{x}_{2}+\left(g / l_{2}\right) x_{2}-\left(g / l_{2}\right) x=0
\end{array}\right.
$$

and may set $\dot{x}_{1}=x_{3}, \dot{x}_{2}=x_{4}$ in order to bring the preceding system to Kalman form with 4 first order OD equations. The controllability condition is then easily seen to be $l_{1} \neq l_{2}$ but such a result not only seems to depend on the choice of input and output but cannot be extended to PD equations.

Problem 4.2: Any engineer knows about the first set of Maxwell equations:

$$
\vec{\nabla} \cdot \vec{B}=0, \quad \vec{\nabla} \wedge \vec{E}+\frac{\partial \vec{B}}{\partial t}=0
$$

and the fact that any solution can be written in the form:

$$
\vec{B}=\vec{\nabla} \wedge \vec{A}, \quad \vec{E}=-\vec{\nabla} \cdot V-\frac{\partial \vec{A}}{\partial t}
$$

for an arbitrary vector $\vec{A}$ and an arbitrary function $V$.

According to special relativity, these equations can be condensed on space-time by introducing a 1 -form $A$ for the potential and a 2 -form $F$ for the field in order to discover that the above Mawell equations can be written in the form $d F=0$ and admit the "generic" solution $d A=F$ where $d$ is the exterior derivative. Hence, we have "parametrized" the field equations by means of a "potential", that is the field equations generate the compatibility conditions of the inhomogeneous system allowing to express the field (right member) by means of the potential (left member).

Similarly, in 2-dimensional elasticity theory, if we want to solve the stress equations with no density of force, namely:

$$
\partial_{1} \sigma^{11}+\partial_{2} \sigma^{21}=0 \quad, \quad \partial_{1} \sigma^{12}+\partial_{2} \sigma^{22}=0
$$

we may use the first $\mathrm{PD}$ equation to get:

$$
\exists \varphi, \quad \sigma^{11}=\partial_{2} \varphi \quad, \quad \sigma^{21}=-\partial_{1} \varphi
$$

and the second $\mathrm{PD}$ equation to get:

$$
\exists \psi, \quad \sigma^{12}=-\partial_{2} \psi \quad, \quad \sigma^{22}=\partial_{1} \psi
$$

Now, $\sigma^{12}=\sigma^{21} \Rightarrow \exists \phi, \varphi=\partial_{2} \phi \quad, \quad \psi=\partial_{1} \phi$

and we finally get the generic parametrization by the Airy function:

$$
\sigma^{11}=\partial_{22} \phi \quad, \quad \sigma^{12}=\sigma^{21}=-\partial_{12} \phi \quad, \quad \sigma^{22}=\partial_{11} \phi
$$

The reader will have noticed that such a specific computation cannot be extended in general, even to 3-dimensional elasticity theory.

In 1970 J. Wheeler asked a similar question for Einstein equations in vacuum and we present the linearized version of this problem.

Indeed, if $\omega=\left(d x^{1}\right)^{2}+\left(d x^{2}\right)^{2}+\left(d x^{3}\right)^{2}-\left(d x^{4}\right)^{2}$ with $x^{4}=c t$, where $c$ is the speed of light, is the Minkowski metric of space-time, we may consider a perturbation $\Omega$ of $\omega$ and the linearized Einstein equations in vacuum become equivalent to the following second order system with 10 equations for 10 unknowns:

$$
\begin{aligned}
& \omega^{r s}\left(d_{i j} \Omega_{r s}+d_{r s} \Omega_{i j}-d_{r i} \Omega_{s j}-d_{s j} \Omega_{r i}\right) \\
& \quad-\omega_{i j}\left(\omega^{r s} \omega^{u v} d_{r s} \Omega_{u v}-\omega^{r u} \omega^{s v} d_{r s} \Omega_{u v}\right)=0
\end{aligned}
$$


Surprisingly, till we gave the (negative) answer in 1995 [17], such a problem had never been solved before.

More generally, if one considers a system of the form $\mathcal{D}_{1} \eta=0$, the question is to know whether one can parametrize or not the solution space by $\mathcal{D} \xi=\eta$ with arbitrary potential-like functions $\xi$, in such a way that $\mathcal{D}_{1} \eta=0$ just generates the compatibility conditions of the parametrization. The problem of multiple parametrizations may also be considered, as an inverse to the construction of differential sequences. For example, in vector calculus, the div operator is parametrized by the curl operator which is itself parametrized by the grad operator (See $[19,21,22]$ for more details).

Problem 4.3: When $M$ is an $A$-module, there is a canonical morphism $\epsilon=\epsilon_{M}: M \longrightarrow M^{* *}$ given by $\epsilon(x)(f)=f(x), \forall x \in M, \forall f \in M^{*}$ and $M$ is said to be torsionless if $\epsilon$ is injective and reflexive if $\epsilon$ is bijective. Any finitely projective module is reflexive but a reflexive module may not be projective. We have $t(M) \subseteq \operatorname{ker}(\epsilon)$ because, if $x \in M$ is a torsion element for $a \neq 0$, then $a f(x)=f(a x)=f(0)=0 \Rightarrow f(x)=0, \forall f \in M^{*}$ as before and $\epsilon$ fails to be injective. Hence, it just remains to study whether this inclusion is strict or not.

The striking result of this lecture is to prove that THESE THREE PROBLEMS ARE IDENTICAL !.

\section{I.5 PROBLEM SOLUTION}

The main but highly not evident trick will be to introduce the adjoint operator $\tilde{\mathcal{D}}=\operatorname{ad}(\mathcal{D})$ by the formula of integration by part:

$$
<\lambda, \mathcal{D} \xi>=<\tilde{\mathcal{D}} \lambda, \xi>+\operatorname{div}(\quad)
$$

where $\lambda$ is a test row vector and $<>$ denotes the usual contraction. The adjoint can also be defined formally, as in computer algebra packages, by setting $a d(a)=a, \forall a \in K, \operatorname{ad}\left(d_{i}\right)=-d_{i}, a d(P Q)=\operatorname{ad}(Q) a d(P), \forall P, Q \in D$. Another way is to define the adjoint of an operator directly on $D$ by setting $P=\sum_{0 \leq|\mu| \leq p} a^{\mu} d_{\mu} \longrightarrow \operatorname{ad}(P)=$ $\sum_{0 \leq|\mu| \leq p}(-1)^{|\mu|} d_{\mu} a^{\mu}$ for any $P \in D$ with $\operatorname{ord}(P)=p$ and to extend such a definition by linearity.

We shall denote by $N$ the differential module defined from $\operatorname{ad}(\mathcal{D})$ exactly like $M$ was defined from $\mathcal{D}$, we have $[7,13,14,19]$ :

Theorem 5.1: The following statements are equivalent:

- A control system is controllable.

- The corresponding operator is simply (doubly) parametrizable.

- The corresponding module is torsion-free (reflexive).

Proof: Let us start with a free presentation of $M$ :

$$
F_{1} \stackrel{d_{1}}{\longrightarrow} F_{0} \longrightarrow M \longrightarrow 0
$$

By definition, we have $M=\operatorname{coker}\left(d_{1}\right) \Longrightarrow N=\operatorname{coker}\left(d_{1}^{*}\right)$ and we may exhibit the following free resolution of $N$ :

$$
0 \longleftarrow N \longleftarrow F_{1}^{*} \stackrel{d_{1}^{*}}{\longleftarrow} F_{0}^{*} \stackrel{d_{0}^{*}}{\longleftarrow} F_{-1}^{*} \stackrel{d_{-1}^{*}}{\longleftarrow} F_{-2}^{*}
$$

where $M^{*}=\operatorname{ker}\left(d_{1}^{*}\right)=\operatorname{im}\left(d_{0}^{*}\right) \simeq \operatorname{coker}\left(d_{-1}^{*}\right)$. The deleted sequence is:

$$
0 \longleftarrow F_{1}^{*} \stackrel{d_{1}^{*}}{\longleftarrow} F_{0}^{*} \stackrel{d_{0}^{*}}{\longleftarrow} F_{-1}^{*} \stackrel{d_{-1}^{*}}{\longleftarrow} F_{-2}^{*}
$$

Applying $\operatorname{hom}_{A}(\bullet, A)$ and using the canonical isomorphism $F^{* *} \simeq F$ for any free module $F$, we get the sequence:

$$
\begin{array}{ccccc}
0 \longrightarrow F_{1} \stackrel{d_{1}}{\longrightarrow} & F_{0} & \stackrel{d_{0}}{\longrightarrow} & F_{-1} & \stackrel{d_{-1}}{\longrightarrow} F_{-2} \\
\downarrow & & \uparrow & \\
M & \stackrel{\epsilon}{\longrightarrow} & M^{* *} \\
& & & \uparrow \\
0 & & 0 &
\end{array}
$$

Denoting as usual a coboundary space by $B$, a cocycle space by $Z$ and the corresponding cohomology by $H=Z / B$, we get the commutative and exact diagram:

$$
\begin{array}{cccccc}
0 \longrightarrow & B_{0} & \longrightarrow & F_{0} & \longrightarrow & M \\
& & & \| & & \downarrow \\
0 & Z_{0} & \longrightarrow & F_{0} & \longrightarrow & M^{* *}
\end{array}
$$


An easy chase provides at once $H_{0}=Z_{0} / B_{0}=\operatorname{ext}_{A}^{1}(N, A) \simeq \operatorname{ker}(\epsilon)$. It follows that $\operatorname{ker}(\epsilon)$ is a torsion module and, as we already know that $t(M) \subseteq \operatorname{ker}(\epsilon) \subseteq M$, we finally obtain $t(M)=k e r(\epsilon)$. Also, as $B_{-1}=i m(\epsilon)$ and $Z_{-1} \simeq M^{* *}$, we obtain $H_{-1}=Z_{-1} / B_{-1}=\operatorname{ext}_{A}^{2}(N, A) \simeq \operatorname{coker}(\epsilon)$. Accordingly, a torsion-free (reflexive) module is described by an operator that admits a single (double) step parametrization.

Q.E.D.

This proof also provides an effective test for applications by using $D$ and ad instead of $A$ and $*$ in the differential framework. In particular, a control system is controllable if it does not admit any "autonomous element", that is to say any finite linear combination of the control variables and their derivatives that satisfies, for itself, at least one $\mathrm{OD}$ or PD equation. More precisely, starting with the control system described by an operator $\mathcal{D}_{1}$, one MUST construct $\tilde{\mathcal{D}}_{1}$ and then $\mathcal{D}$ such that $\tilde{\mathcal{D}}$ generates all the compatibility conditions of $\tilde{\mathcal{D}}_{1}$. Finally, $M$ is torsion-free if and only if $\mathcal{D}_{1}$ generates all the compatibility conditions of $\mathcal{D}$. Though striking it could be, this is the true generalization of the standard Kalman test.

Example 5.2: If $\mathcal{D}_{1}:\left(\sigma^{11}, \sigma^{12}=\sigma^{21}, \sigma^{22}\right) \rightarrow\left(\partial_{1} \sigma^{11}+\partial_{2} \sigma^{21}, \partial_{1} \sigma^{12}+\partial_{2} \sigma^{22}\right)$ is the stress operator, then $\tilde{\mathcal{D}}_{1}:\left(\xi^{1}, \xi^{2}\right) \rightarrow\left(\partial_{1} \xi^{1}=\epsilon_{11}, \frac{1}{2}\left(\partial_{1} \xi^{2}+\partial_{2} \xi^{1}\right)=\epsilon_{12}=\epsilon_{21}, \partial_{2} \xi^{2}=\epsilon_{22}\right)$ is half of the Killing operator. The only compatibility condition for the strain tensor $\epsilon$ is $\tilde{\mathcal{D}} \epsilon=0 \Leftrightarrow \partial_{11} \epsilon_{22}+\partial_{22} \epsilon_{11}-2 \partial_{12} \epsilon_{12}=0$ and $\mathcal{D}$ describes the Airy parametrization.

Now, in order to have a full picture of the correspondence existing between differential modules and differential operators, it just remains to explain why and how we can pass from left to right modules and conversely. By this way, we shall be able to take into account the behaviour of the adjoint of an operator under changes of coordinates. We start with a technical lemma (exercise):

Lemma 5.3: If $f \in \operatorname{aut}(X)$ is a local diffeomorphism of $X$, we may set $y=f(x) \Rightarrow x=f^{-1}(y)=g(y)$ and introduce the jacobian $\Delta(x)=\operatorname{det}\left(\partial_{i} f^{k}(x)\right) \neq 0$. Then, we have the identity:

$$
\frac{\partial}{\partial y^{k}}\left(\frac{1}{\Delta(g(y))} \partial_{i} f^{k}(g(y)) \equiv 0\right. \text {. }
$$

Accordingly, we notice that, if $\mathcal{D}: E \rightarrow F$ is an operator, the way to obtain the adjoint through an integration by parts proves that the test function is indeed a section of the adjoint bundle $\tilde{F}=F^{*} \otimes \Lambda^{n} T^{*}$ and that we get an operator $\operatorname{ad}(\mathcal{D}): \tilde{F} \rightarrow \tilde{E}$. This is in particular the reason why, in elasticity, the deformation is a covariant tensor but the stress is a contravariant tensor density and, in electromagnetism, the EM field is a covariant tensor (in fact a 2-form) but the induction is a contravariant tensor density.

Also, if we define the adjoint formally, we get, in the operator sense:

$$
a d\left(\frac{1}{\Delta} \partial_{i} f^{k} \frac{\partial}{\partial y^{k}}\right)=-\frac{\partial}{\partial y^{k}} \circ\left(\frac{1}{\Delta} \partial_{i} f^{k}\right)=-\frac{1}{\Delta} \partial_{i} f^{k} \frac{\partial}{\partial y^{k}}=-\frac{1}{\Delta} \frac{\partial}{\partial x^{i}}
$$

and obtain therefore:

$$
\frac{\partial}{\partial x^{i}}=\partial_{i} f^{k}(x) \frac{\partial}{\partial y^{k}} \Rightarrow a d\left(\frac{\partial}{\partial x^{i}}\right)=-\frac{\partial}{\partial x^{i}}=\Delta a d\left(\frac{1}{\Delta} \partial_{i} f^{k}(x) \frac{\partial}{\partial y^{k}}\right)
$$

a result showing that the adjoint of the gradient operator $d: \Lambda^{0} T^{*} \rightarrow \Lambda^{1} T^{*}$ is minus the exterior derivative $d: \Lambda^{n-1} T^{*} \rightarrow \Lambda^{n} T^{*}$.

If $A$ is a differential ring and $D=A[d]$ as usual, we may introduce the ideal $I=\{P \in D \mid P(1)=0\}$ and obtain $A \simeq D / I$ both with the direct sum decomposition $D \simeq A \oplus I$. In fact, denoting by $D_{q}$ the submodule over $A$ of operators of order $q, A$ can be identified with the subring $D_{0} \subset D$ of zero order operators and we may consider any differential module over $D$ as a module over $A$, just "forgetting" about its differential structure. Caring about the notation, we shall set $T=D_{1} / D_{0}=\left\{\xi=a^{i} d_{i} \mid a^{i} \in A\right\}$ with $\xi(a)=\xi^{i} \partial_{i} a, \forall a \in A$, so that $D$ can be generated by $A$ and $T$.

The module counterpart is much more tricky and is based on the following theorem [19]:

Theorem 5.4: If $M$ and $N$ are right $D$-modules, then $\operatorname{hom}_{A}(M, N)$ becomes a left $D$-module. 
Proof: We just need to define the action of $\xi \in T$ by the formula:

$$
(\xi f)(m)=f(m \xi)-f(m) \xi, \quad \forall m \in M
$$

Indeed, setting $(a f)(m)=f(m) a=f(m a)$ and introducing the bracket $(\xi, \eta) \rightarrow[\xi, \eta]$ of vector fields, we let the reader check that $a(b f)=(a b) f, \forall a, b \in A$ and that we have the formulas:

$$
\xi(a f)=(\xi(a)+a \xi) f, \quad(\xi \eta-\eta \xi) f=[\xi, \eta] f, \forall a \in A, \forall \xi, \eta \in T
$$

in the operator sense.

Q.E.D.

Finally, if $M$ is a left $D$-module, according to the comment following lemma 3.1.13, then $M^{*}=h o m_{D}(M, D)$ is a right $D$-module and thus $N=N_{r}$ is a right $D$-module. However, we have the following technical proposition:

Proposition 5.5: $\Lambda^{n} T^{*}$ has a natural right module structure over $D$.

Proof: If $\alpha=a d x^{1} \wedge \ldots \wedge d x^{n} \in T^{*}$ is a volume form with coefficient $a \in A$, we may set $\alpha . P=a d(P)(a) d x^{1} \wedge \ldots \wedge d x^{n}$. As $D$ is generated by $A$ and $T$, we just need to check that the above formula has an intrinsic meaning for any $\xi \in T$. In that case, we check at once:

$$
\alpha . \xi=-\partial_{i}\left(a \xi^{i}\right) d x^{1} \wedge \ldots \wedge d x^{n}=-\mathcal{L}(\xi) \alpha
$$

by introducing the Lie derivative of $\alpha$ with respect to $\xi$, along the intrinsic formula $\mathcal{L}(\xi)=i(\xi) d+d i(\xi)$ where $i()$ is the interior multiplication and $d$ is the exterior derivative of exterior forms. According to well known properties of the Lie derivative, we get :

$$
\alpha \cdot(a \xi)=(\alpha . \xi) . a-\alpha . \xi(a), \quad \alpha \cdot(\xi \eta-\eta \xi)=-[\mathcal{L}(\xi), \mathcal{L}(\eta)] \alpha=-\mathcal{L}([\xi, \eta]) \alpha=\alpha .[\xi, \eta] .
$$

Q.E.D.

According to the preceding theorem and proposition, the left differential module corresponding to $\operatorname{ad}(\mathcal{D})$ is not $N_{r}$ but rather $N_{l}=\operatorname{hom}_{A}\left(\Lambda^{n} T^{*}, N_{r}\right)$. When $D$ is a commutative ring, this side changing procedure is no longer needed.

Of course, keeping the same module $M$ but changing its presentation or even using an isomorphic module $M^{\prime}$ (2 OD equations of order 2 or 4 OD equations of order 1 as in the case of the double pendulum), then $N$ may

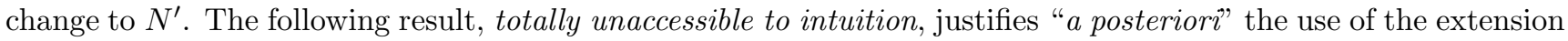
functor by proving that the above results are unchanged and are thus "intrinsic" [19,22].

Theorem 5.6: $N$ and $N^{\prime}$ are projectively equivalent, that is to say one can find projective modules $P$ and $P^{\prime}$ such that $N \oplus P \simeq N^{\prime} \oplus P^{\prime}$.

Proof: According to Schanuel lemma, we can always suppose, with no loss of generality, that the resolution of $M$ projects onto the resolution of $M^{\prime}$. The kernel sequence is a splitting sequence made up with projective modules because the kernel of the projection of $F_{i}$ onto $F_{i}^{\prime}$ is a projective module $P_{i}$ for $i=0,1$. Such a property still holds when applying duality. Hence, if $C$ is the kernel of the epimorphism from $P_{1}$ to $P_{0}$ induced by $d_{1}$, then $C$ is a projective module and the top short exact sequence splits in the following commutative and exact diagram:

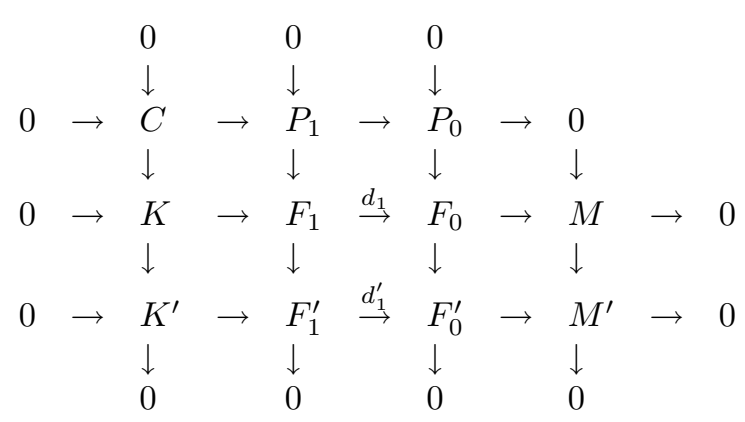


Applying $\operatorname{hom}_{A}(\bullet, A)$ to this siagram while taking into account Corollary 3.1.15, we get the following commutative and exact diagram:

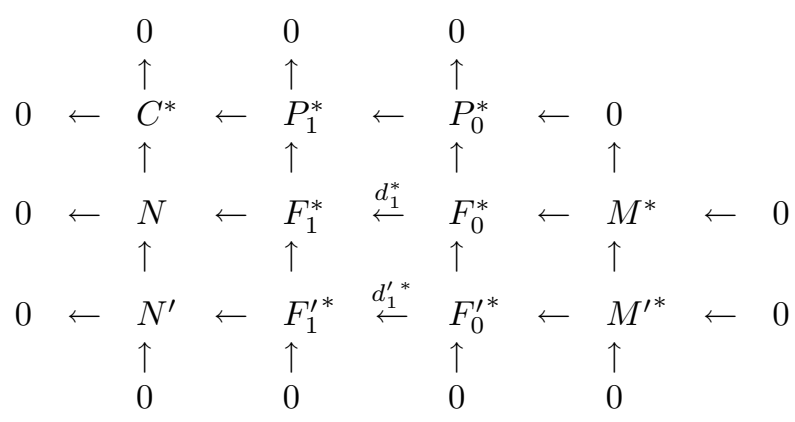

In this diagram $C^{*}$ is also a projective module, the upper and left short exact sequences split and we obtain $N \simeq N^{\prime} \oplus C^{*}$.

Q.E.D.

Remark 5.7: When $A$ is a principal ideal ring, it is well known (See [3,23] for more details) that any torsion-free module over $A$ is free and thus projective. Accordingly, the kernel of the projection of $F_{0}$ onto $M$ is free and we can always suppose, with no loss of generality, that $d_{1}$ and $d_{1}^{\prime}$ are monomorphisms [8]. In that case, there is an isomorphism $P_{0} \simeq P_{1}$ in the proof of the preceding theorem and $C=0 \Rightarrow C^{*}=0$, that is to say $N \simeq N^{\prime}$. This is the very specific situation only considered by OD control theory where the OD equations defining the control systems are always supposed to be differentially independent (linearly independent over $D$ ).

Accordingly, using the properties of the extension functor, we get:

Corollary 5.8: $\operatorname{ext}_{A}^{i}(N, A) \simeq \operatorname{ext}_{A}^{i}\left(N^{\prime}, A\right) \quad \forall i \geq 1$.

We finally apply these results in order to solve the three preceding problems.

Solution 5.9: As the operator $\mathcal{D}$ of the control system is surjective, it follows that the map $d_{1}$ of the presentation is injective. When $K=\mathbb{R}$ and $n=1$, then $D$ can be identified with a polynomial ring in one indeterminate and is therefore a principal ideal domain (any ideal can be generated by a single polynomial). In this case, it is well known, as we just said, that any torsion-free module is indeed free and thus projective. The short exact sequence of the presentation splits, with a similar comment for its dual sequence. Accordingly, $M$ is torsion-free if and only if $N=0$ and it just remains to prove that $\tilde{\mathcal{D}}$ is injective. We have to solve the system:

$$
\left\{\begin{array}{c}
x \longrightarrow \ddot{\lambda}_{1}+\ddot{\lambda}_{2}=0 \\
\theta_{1} \longrightarrow l_{1} \ddot{\lambda}_{1}+g \lambda_{1}=0 \\
\theta_{2} \longrightarrow l_{2} \ddot{\lambda}_{2}+g \lambda_{2}=0
\end{array}\right.
$$

Multiplying the second OD equation by $l_{2}$, the third by $l_{1}$ and adding them while taking into account the first OD equation, we get:

$$
l_{2} \lambda_{1}+l_{1} \lambda_{2}=0
$$

Differentiating this OD equation twice while using the second and third OD equations, we get:

$$
\left(l_{2} / l_{1}\right) \lambda_{1}+\left(l_{1} / l_{2}\right) \lambda_{2}+0
$$

The determinant of this linear system for $\lambda_{1}$ and $\lambda_{2}$ is just $l_{1}-l_{2}$, hence the system is controllable if and only if $l_{1} \neq l_{2}$.

Conversely, if $l_{1}=l_{2}=l$, the corresponding module has torsion elements. In particular, setting $\theta=\theta_{1}-\theta_{2}$ and substracting the second dynamic equation from the first, we get $l \ddot{\theta}+g \theta=0$. Hence $\theta$ is a torsion element which is solution of an autonomous OD equation, that is an OD equation for itself which cannot therefore be "controlled" by any means.

We now study the problem of a double-pendulum when one of the pendula has a variable length, namely , setting $l_{1}=l(t), l_{2}=1, g=1$, we study the system:

$$
\ddot{x}+l(t) \ddot{\theta}_{1}+\theta_{1}=0, \quad \ddot{x}+\ddot{\theta}_{2}+\theta_{2}=0
$$


Multiplying these two OD equations by test functions $\lambda^{1}, \lambda^{2}$ and integrating by part, we obtain for the kernel of the adjoint operator:

$$
\ddot{\lambda}^{1}+\ddot{\lambda}_{2}=0, \quad l \ddot{\lambda}^{1}+2 \ddot{\lambda}^{1}+(\ddot{l}+1) \lambda^{1}=0, \quad \ddot{\lambda}^{2}+\lambda^{2}=0
$$

Eliminating $\lambda^{2}$ among the first and third OD equations, we get at once for $\lambda=\lambda^{1}$ the system:

$$
\lambda^{(4)}+\ddot{\lambda}=0, \quad l \ddot{\lambda}+2 \ddot{l} \dot{\lambda}+(\ddot{l}+1) \lambda=0
$$

which is surely not formally integrable.

Differentiating twice the second OD equation, we successively get:

$$
\begin{gathered}
l \lambda^{(3)}+3 \ddot{\lambda}+(3 \ddot{l}+1) \dot{\lambda}+l^{(3)} \lambda=0 \\
l \lambda^{(4)}+4 \dot{l} \lambda^{(3)}+(6 \ddot{l}+1) \ddot{\lambda}+4 l^{(3)} \dot{\lambda}+l^{(4)} \lambda=0
\end{gathered}
$$

Using the first OD equation of order 4, we get:

$$
4 \dot{l} \lambda^{(3)}+(6 \ddot{l}-l+1) \ddot{\lambda}+4 l^{(3)} \dot{\lambda}+l^{(4)} \lambda=0
$$

Using the previous OD equation of order 3, we get:

$$
\left(6 l \ddot{l}-12 \dot{l}^{2}-l^{2}+l\right) \ddot{\lambda}+\left(4 l l^{(3)}-12 \dddot{l}-4 i\right) \dot{\lambda}+\left(l l^{(4)}-4 l^{(3)}\right) \lambda=0
$$

Using the previous OD equation of order 2, we get:

$$
\left(4 l^{2} l^{(3)}-24 l \dddot{l} \ddot{l}-6 l \dot{l}+2 l^{2} \dot{l}+24 \dot{l}^{3}\right) \dot{\lambda}+\left(l^{2} l^{(4)}-4 l \dot{l}^{(3)}-(\ddot{l}+1)\left(6 l \ddot{l}-4 \dot{l}^{2}-l^{2}+l\right)\right) \lambda=0
$$

It remains to differentiate this OD equation, substitute the $\ddot{\lambda}$ obtained from the first OD equation of order 2 found for $\lambda$ and eliminate $\dot{\lambda}, \lambda$ in the resulting linear system of two OD equations by equating to zero the corresponding determinant. We should obtain for $l(t)$ A HIGHLY NONLINEAR OD EQUATION OF ORDER 5. The MAPLE output has been produced by Daniel Robertz (daniel@momo.math.rwth-aachen.de) and covers half a page!.

Of course, no computer algebra technique may work in order to look for all the solutions of this OD equation or even have any idea of possible solutions. By chance, in this specific case, exactly as in [19, Example 1.104 where one should point out a confusion as the adjoint operator corresponds to the reduced Kalman type system with variable length and not to the bipendulum, though the conceptual approach is similar], there is a way to find out directly the general solution by integrating the system for $\lambda^{1}=\lambda, \lambda^{2}$ and $l$. From the third OD equation, we get:

$$
\lambda^{2}=\alpha \cos t+\beta \sin t
$$

where $\alpha$ and $\beta$ are arbitrary constants and thus, from the first OD equation, we therefore obtain:

$$
\lambda=a t+b-\alpha \cos t-\beta \sin t
$$

where $a$ and $b$ are again arbitrary constants. Accordingly, we get:

$$
\left(d^{2} / d t^{2}\right)(l \lambda)=-\lambda=-a t-b+\alpha \cos t+\beta \sin t
$$

and thus, with two new arbitrary constants $c$ and $d$, we get:

$$
l \lambda=-\frac{1}{6} a t^{3}-\frac{1}{2} b t^{2}+c t+d-\alpha \cos t-\beta \sin t
$$

We finally get the general solution:

$$
l(t)=\frac{-\frac{1}{6} a t^{3}-\frac{1}{2} b t^{2}+c t+d-\alpha \cos t-\beta \sin t}{a t+b-\alpha \cos t-\beta \sin t}
$$

For example, if $a=b=0$, we get:

$$
l(t)=1-\frac{c t+d}{\alpha \cos t+\beta \sin t}
$$

but other possibilities may be of interest and could be tested or simulated, provided $l(t) \geq 0$. Of course, if $l(t)=1$, we get $a=b=c=d=0 \Rightarrow \lambda^{1}+\lambda^{2}=0$ while, if $l(t)=c s t \neq 1$, we also get $\alpha=\beta=0 \Rightarrow \lambda^{1}=\lambda^{2}=0$ in a 
coherent way with the constant coefficient case.

Solution 5.10: After a short computation left to the reader as an exercise, one checks easily that the Einstein operator is self-adjoint because the 6 terms are just exchanged between themselves. Then, it is well known that the compatibility condition is made by the standard divergence operator and its adjoint is the Killing operator (Lie derivative of the Minkowski metric) which admits the linearized Riemann curvature (20 PD equations) as compatibility conditions and not the Einstein equations (10 PD equations only). Hence, the Einstein operator cannot be parametrizable and it follows that Einstein equations cannot be any longer considered as field equations (For a computer algebra solution, see [27]).

Solution 5.11: It has already been provided by the preceding theorems.

Remark 5.12: Writing a Kalman type system in the form $-\dot{x}+A x+B u=0$ and multiplying on the left by a test row vector $\lambda$, the kernel of the adjoint operator is defined by the system:

$$
\dot{\lambda}+\lambda A=0, \quad \lambda B=0
$$

Differentiating the second equations, we get:

$$
\dot{\lambda} B=0 \Longrightarrow \lambda A B=0 \Longrightarrow \lambda A^{2} B=0 \Longrightarrow \ldots
$$

and we discover that the Kalman criterion just amounts to the injectivity of the adjoint operator. Hence, in any case, controllability only depends on formal integrability. Comparing with the Motivating Examples, we notice that, when a constant coefficient operator is injective, the fact that we can find differentially independent compatibility conditions is equivalent to the Quillen-Suslin theorem saying roughly that a projective module over a polynomial ring is indeed free (See [9,23] for details). More generally, by duality we obtain at once $t(M) \simeq \operatorname{ext}_{A}^{1}(N, A) \Leftrightarrow t(N) \simeq \operatorname{ext}_{A}^{1}(M, A)$ and this result is coherent with the introduction of this lecture provided we say that a control system is "observable" if $\operatorname{ext}_{A}^{1}(M, A)=0$. Finally, in the case of the linearized system with variable coefficients provided at the end of section 2 , multilying by a test function $\lambda$ and integrating by parts, we have to study the system :

$$
U \longrightarrow \dot{\lambda}+\dot{y} \lambda=0, \quad Y \longrightarrow u \dot{\lambda}+\dot{u} \lambda=0 .
$$

Multiplying the first OD equation by $u$ and substracting the second, we get $a \lambda=0$ and the linearized system is controllable if and only if $a \neq 0$.

We now explain the link existing between localization and parametrization [21]. This result will prove at once that localization is the good substitute for superseding the transfer matrix approach and can even be used for systems with variable coefficients. The main idea is contained in the following technical proposition:

Proposition 5.15: If $A$ is an integral domain with quotient field $K=Q(A)$ and $M$ is a finitely generated torsionfree module over $A$, then $M$ can be embedded into a free module of the same rank.

Proof: As $t(M)=0$, the module $M=\left(x_{1}, \ldots, x_{n}\right)$ can be identified with a submodule of $K \otimes_{A} M$. Also, as $K \otimes_{A} M$ is a vector space over $K$, the $x_{1}, \ldots, x_{n}$ may not be lineary independent over $K$ and we may select a basis $\left(e_{1}, \ldots, e_{m}\right)$ with $r k_{A}(M)=m \leq n$. We have therefore $x_{i}=\sum_{j=1}^{m} b_{i}^{j} e_{j}$ with $b_{i}^{j} \in K$. We may reduce all these coefficients to the same denominator $s \in S=A-\{0\}$ and write $x_{i}=\sum \frac{a_{i}^{j}}{s} e_{i}=\sum a_{i}^{j} \bar{e}_{j}$ with $a_{i}^{j} \in A$. Accordingly, $M$ becomes a submodule of the free module generated over $A$ by the $\bar{e}$ which are linearly independent over $K$ and thus over $A$.

Q.E.D.

When $D$ is used in place of $A$ with $S=D-\{0\}$, the expression $x_{i}=\sum_{j=1}^{m} a_{i}^{j} \bar{e}_{j}$ just describes the parametrization of the corresponding system by $m$ potentials. In the non-commutative case, the technique relies on the following lemma that essentially uses the adjoint operator:

Lemma 5.16: $S^{-1} D \simeq D S^{-1}$.

Proof: Let $U \in S$ and $P \in D$. We may consider the adjoint operators $\tilde{U}=\operatorname{ad}(U) \in S$ and $\tilde{P}=\operatorname{ad}(P) \in D$. Taking into account the Ore property of $S \subset D$, we may find $\tilde{V}=\operatorname{ad}(V) \in S$ and $\tilde{Q}=\operatorname{ad}(Q) \in D$ such that $\tilde{V} \tilde{P}=\tilde{Q} \tilde{U}$ 
and obtain therefore $P V=U Q$, that is to say $U^{-1} P=Q V^{-1}$.

Q.E.D.

Example 5.17: With $n=2, m=2, K=\mathbb{Q}\left(x^{1}, x^{2}\right)$, let us consider the system $d_{1} y^{1}-d_{2} y^{2}-x^{2} y^{1}=0$. We have $d_{2} y^{2}=d_{1} y^{1}-x^{2} y^{1}$ and thus $y^{2}=d_{2}^{-1}\left(d_{1}-x^{2}\right) y^{1}$. Using the above lemma, we obtain (exercise) the identity:

$$
\left(d_{1}-x^{2}\right) d_{22}=d_{2}\left(d_{12}-x^{2} d_{2}+1\right)
$$

We have therefore $y^{2}=\left(d_{12}-x^{2} d_{2}+1\right) d_{22}^{-1} y^{1}$. Hence, setting $d_{22}^{-1} y^{1}=z$, we get the parametrization:

$$
y^{1}=d_{22} z, \quad y^{2}=\left(d_{12}-x^{2} d_{2}+1\right) z
$$

This parametrization is of course not unique and one could exhibit the other (very different) parametrization:

$$
y^{1}=\left(d_{12}-x^{2} d_{2}-2\right) z, \quad y^{2}=\left(d_{11}-2 x^{2} d_{1}+\left(x^{2}\right)^{2}\right) z
$$

In both cases, we check that the corresponding compatibility conditions are indeed generated by the given system.

We have therefore exhibited a kind of non-commutative SISO transfer function for a system with variable coefficients. In actual practice, unless we know by means of other techniques that $M$ is torsion-free, the only way to look for a parametrization is to proceed as above for exhibiting one parametrization and to check that it is indeed generic. Of course, many other different parametrizations may exist as in the above example.

At the end of this section, we shall present a new constructive way to study the torsion submodule $t(M)$ of a differential module $M$ having torsion.

For this, setting $D^{\prime}=K\left[d_{1}, \ldots, d_{n-1}\right] \subset D$, let us now introduce the nested chain:

$$
M_{(0)} \subseteq M_{(1)} \subseteq \ldots \subseteq M_{(\infty)}=M
$$

of $D^{\prime}$-submodules of the $D$-module $M$ by defining $M_{(r)}$ as the residue of the set of all parametric jets for which $y_{\mu}^{k}$ is such that $1 \leq k \leq \beta_{1}^{n}, \mu_{n}=0$ and $z_{\nu}^{l}$ is such that $1 \leq l \leq m-\beta_{1}^{n}, \nu_{n} \leq r$.

Definition 5.18: We shall introduce the chain of $D^{\prime}$-submodules:

$$
M_{(-\infty)} \subseteq \ldots \subseteq M_{(-1)} \subseteq M_{(0)}
$$

by setting $M_{(-r-1)}=\left\{m \in M_{(-r)} \mid d_{n} m \in M_{(-r)}\right\}$.

Our main result is the following theorem (compare to [ ]):

Theorem 5.19: $M_{(-\infty)}=t(M)$.

Proof: By analogy with the method used for the Kalman form, a torsion element cannot contain any $z_{\nu}$ with $\nu_{n} \geq 1$ and thus $t(M) \subset M_{(0)}$. As $t(M)$ is a $D$-module, it is stable by $d_{n}$ and $t(M) \subseteq M_{(-\infty)}$. Let now $w \in M_{(-\infty)}$ be an element of order $q$. The number of derivatives of $w$ of order $r$ is a polynomial in $r$ with leading term $\frac{1}{n !} r^{n}$. Let us now call jet of class $i$ any jet with $\mu_{1}=\ldots=\mu_{i-1}=0$ and equation of class $i$ any equation solved with respect to a leading jet of class $i$, called principal. We notice that the prolongations of an involutive solved form are also in involutive solved form because the prolongation of an equation of class $i$ with respect to a multiplicative variable $x^{j}$ becomes an equation of class $j$, as $j \leq i$. Accordingly, one can express all the principal jets as linear combinations of the parametric jets and the number of such jets of order $q+r$ for each dependent variable is a polynomial in $r$ with leading term not greater than $\frac{1}{(n-1) !} r^{n-1}$. Hence, when $r$ is large enough, one can eliminate the parametric jets among the derivatives of $w$ that must therefore satisfy at least one OD or PD equation for itself, that is to say $M_{(-\infty)} \subseteq t(M)$.

Q.E.D.

Remark 5.20: Using $(\bar{y}, z)$ in place of $(y, z)$ as we did for the Kalman form, we discover that a torsion element cannot contain anymore $z$ or its jets and only depends on $\bar{y}$ and its jets with respect to $x^{1}, \ldots, x^{n-1}$. The Kalman form is thus only the particular case $n=1$ of the modified Spencer form. In this case, $M_{(-1)}=$ $\{\Sigma \lambda x\} \subset M_{(0)}=\{\Sigma \lambda x+\Sigma \mu u\}$ and $M_{(-r-2)}$ can be identified with the orthogonal space to $S_{r}$ in $M_{(-1)}$ with 
$\operatorname{dim}\left(M_{(-r-2)}\right)=n-\operatorname{dim}\left(S_{r}\right)$ for $r \geq 0$.

Example 5.21: Looking back to example [19], we notice that $M_{(0)}$ is generated by $\left(\bar{y}^{1}, \bar{y}^{2}, z\right)$ and their jets in $x^{1}$, modulo the only equation $\bar{y}_{1}^{1}-\bar{y}_{1}^{2}=0$ of class 1 . However, $d_{2} z \in M_{(0)}$ and thus $M_{(-1)}$ is only generated by $\left(\bar{y}^{1}, \bar{y}^{2}\right)$. Finally, $t(M)$ is generated by $w=\bar{y}^{1}-\bar{y}^{2}=y^{1}-y^{2}-2 z$ which satisfies $d_{2} w=0$ but also $d_{1} w=0$ and we get $t(M)=M_{(-2)}$.

The effectivity of this recursive algorithm lies in the following non-trivial corollary which constitutes the core of the procedure and brings a quite new understanding of the controllability indices (Compare to [1]).

Corollary 5.22: $t(M)=M_{(-r)}$ for a certain $r<\infty$.

Proof: The previous definitions amount to the exact sequences:

$$
0 \longrightarrow M_{(-r-1)} \longrightarrow M_{(-r)} \stackrel{d_{n}}{\longrightarrow} M_{(-r+1)} / M_{(-r)}
$$

and we obtain therefore the induced monomorphisms:

$$
0 \longrightarrow M_{(-r)} / M_{(-r-1)} \stackrel{d_{n}}{\longrightarrow} M_{(-r+1)} / M_{(-r)}
$$

of $D^{\prime}$-modules. Composing these monomorphisms, we deduce that all these quotient modules are torsion-free $D^{\prime}$-modules because they are submodules of $M_{(0)} / M_{(-1)}$ which is only generated by the $z$, exactly like in classical control theory.

Now, if $F \simeq D^{r}$ is a free $D$-module, we define its differential rank to be $r k_{D}(F)=r$ and, more generally, if $M$ is a $D$-module, we define its differential rank $r k_{D}(M)$ to be the differential rank of a maximum free differential submodule $F \subseteq M$, and, in this case, $T=M / F$ is a torsion module. In our situation, setting $r k_{D^{\prime}}\left(M_{(-r-1)} / M_{(-r-2)}\right)=l_{r}$, we notice that, if $l_{r}=0$, then $M_{(-r-1)} / M_{(-r-2)}$ is a torsion $D^{\prime}$-module and get a contradiction unless $M_{(-r-1)}=M_{(-r-2)}$. Otherwise, if $l_{r}>0$ strictly, using the additivity property of the differential rank, we obtain the strict inequalities $r k_{D^{\prime}}\left(M_{(-r-2)}\right)<r k_{D^{\prime}}\left(M_{(-r-1)}\right)<r k_{D^{\prime}}\left(M_{(-r)}\right)<\ldots<r k_{D^{\prime}}\left(M_{(-1)}\right)=$ $n b$ of $\bar{y}-n b$ of equations of class $(n-1)<\infty$ and the inductive procedure necessarily stops after a finite number of steps. When $n=1$, the $l_{r}$ are nothing else but the controllability indices and $\bar{y}$ is just the output/state.

Q.E.D.

\section{I.6 POLES AND ZEROS}

In order to explain our motivation in this section, let us start with a simple motivating example and consider the SISO system:

$$
\ddot{y}-3 \dot{y}+2 y=\ddot{u}-\dot{u}
$$

Using a Laplace transform, we should get:

$$
(s-1)(s-2) \hat{y}=s(s-1) \hat{u} \Rightarrow \hat{y}=\frac{s}{s-2} \hat{u}
$$

as we can divide both members by $s-1$. In the rational transfer function thus obtained, we may pay attention separately to the numerator $s$ or to the denominator $s-2$, which are both polynomials in $s$, and look for their roots $s=0$ or $s=2$ respectively called zeros and poles. Of couse, in the present traditional setting, we may obtain $\hat{y}$ from $\hat{u}$ and thus $y$ from $u$ but we could also, asking for the inverse problem, try to obtain $\hat{u}$ from $\hat{y}$ and thus $u$ from $y$. Meanwhile, the common factor $s-1$ with root $s=1$ just "disappeared", though, in view of the way to obtain the transfer function, it could be interpreted either as a zero or as a pole.

It becomes clear that the challenge is now to extend these concepts of poles and zeros to MIMO systems defined by PD equations in a way that should not depend on any multivariable Laplace transform and could be applied to systems with variable coefficients. Also, even if we understand at once that poles and zeros do not any longer, as in the previous sections, depend on a structural property of the control system but rather on an inpututput property, we should like to define poles and zeros in a unique intrinsic way related to module theory.

For this, we need a few more definitions and results on modules that were not needed in the previous sections. 
If $M$ is a module over a $\operatorname{ring} A$ and $\operatorname{ann}(M)=a n n_{A}(M)$ is the annihilator of $M$ in $A$, it follows from its definition that, in any case, $\operatorname{ann}(M)=0$ if $M$ is not a torsion module. Also, if $M^{\prime}$ is a submodule of $M$, then $\operatorname{ann}(M) \subseteq \operatorname{ann}\left(M^{\prime}\right)$. The proof of the following lemma is left to the reader as an exercise:

Lemma 6.1: If $M^{\prime}$ and $M^{\prime \prime}$ are submodules of $M$, we have:

- $\quad \operatorname{ann}\left(M^{\prime}+M^{\prime \prime}\right)=\operatorname{ann}\left(M^{\prime}\right) \cap \operatorname{ann}\left(M^{\prime \prime}\right)$

- $\quad \operatorname{ann}\left(M^{\prime} \oplus M^{\prime \prime}\right)=\operatorname{ann}\left(M^{\prime}\right) \cap \operatorname{ann}\left(M^{\prime \prime}\right)$

- $\quad \operatorname{ann}\left(M^{\prime} \cap M^{\prime \prime}\right) \supseteq \operatorname{ann}\left(M^{\prime}\right)+\operatorname{ann}\left(M^{\prime \prime}\right)$

The key result is the following proposition:

Proposition 6.2: For any short exact sequence:

$$
0 \rightarrow M^{\prime} \stackrel{f}{\rightarrow} M \stackrel{g}{\rightarrow} M^{\prime \prime} \rightarrow 0
$$

one has the relations:

$$
\operatorname{ann}(M) \subseteq \operatorname{ann}\left(M^{\prime}\right) \cap \operatorname{ann}\left(M^{\prime \prime}\right) \Rightarrow \operatorname{rad}(\operatorname{ann}(M))=\operatorname{rad}\left(\operatorname{ann}\left(M^{\prime}\right)\right) \cap \operatorname{rad}\left(\operatorname{ann}\left(M^{\prime \prime}\right)\right)
$$

Proof: If $a \in \operatorname{ann}(M)$, then $f\left(a x^{\prime}\right)=a f\left(x^{\prime}\right)=0, \forall x^{\prime} \in M^{\prime}$ because $x=f\left(x^{\prime}\right) \in M$ and thus ax $=0$ because $f$ is a monomorphism. It follows that $\operatorname{ann}(M) \subset$ ann $\left(M^{\prime}\right)$. Now, if $x^{\prime \prime} \in M^{\prime \prime}$, we have $x^{\prime \prime}=g(x)$ for some $x \in M$ because $g$ is an epimorphism and we get $a x^{\prime \prime}=a g(x)=g(a x)=g(0)=0$, that is $\operatorname{ann}(M) \subseteq \operatorname{ann}\left(M^{\prime \prime}\right)$. It follows that $\operatorname{ann}(M) \subseteq \operatorname{ann}\left(M^{\prime}\right) \cap \operatorname{ann}\left(M^{\prime \prime}\right)$ and thus $\operatorname{rad}(\operatorname{ann}(M)) \subseteq \operatorname{rad}\left(\operatorname{ann}\left(M^{\prime}\right)\right) \cap \operatorname{rad}\left(\operatorname{ann}\left(M^{\prime \prime}\right)\right)$.

Conversely, let $a \in \operatorname{rad}\left(\operatorname{ann}\left(M^{\prime}\right)\right) \cap \operatorname{rad}\left(\operatorname{ann}\left(M^{\prime \prime}\right)\right)$. As $a \in \operatorname{rad}\left(\operatorname{ann}\left(M^{\prime \prime}\right)\right)$, we may find $x \in M$ such that $g(x)=x^{\prime \prime}$ and we have $a^{r} x^{\prime \prime}=0$ for a certain $r \in \mathbb{N}$, that is $a^{r} g(x)=g\left(a^{r} x\right)=0$. Hence we may find $x^{\prime} \in M^{\prime}$ such that $a^{r} x=f\left(x^{\prime}\right)$ because the sequence is exact. As $a \in \operatorname{rad}\left(\operatorname{ann}\left(M^{\prime}\right)\right)$, we have $a^{s} x^{\prime}=0$ for a certain $s \in \mathbb{N}$ and we get $a^{r+s} x=a^{s} a^{r} x=a^{s} f\left(x^{\prime}\right)=f\left(a^{s} x^{\prime}\right)=0$, that is $a \in \operatorname{rad}(\operatorname{ann}(M))$, a result leading to the converse inclusion $\operatorname{rad}\left(\operatorname{ann}\left(M^{\prime}\right)\right) \cap \operatorname{rad}\left(\operatorname{ann}\left(M^{\prime \prime}\right)\right) \subseteq \operatorname{rad}(\operatorname{ann}(M))$.

Q.E.D

Definition 6.3: For any ideal $\mathfrak{a} \in A$, we may introduce the zero of $\mathfrak{a}$ to be the family $Z(\mathfrak{a})=\{\mathfrak{p} \in \operatorname{spec}(A) \mid \mathfrak{p} \supseteq$ $\operatorname{rad}(\mathfrak{a}) \supseteq \mathfrak{a}\}$ of proper prime ideals of $A$.

Definition 6.4: If $\mathfrak{p} \in \operatorname{spec}(A)$, the localization with respect to the multiplicatively closed set $S=A-\mathfrak{p}$ is denoted by $M_{\mathfrak{p}}$ and the support of $M$ is the family $\operatorname{supp}(M)$ of proper prime ideals $\mathfrak{p} \in \operatorname{spec}(A)$ such that $M_{\mathfrak{p}} \neq 0$.

Proposition 6.5: If $M$ is a finitely generated $A$-module, then $\operatorname{supp}(M)=Z(\operatorname{ann}(M))$.

Proof: If $\mathfrak{p} \in \operatorname{spec}(A), \mathfrak{p} \nsupseteq \operatorname{ann}(M)$, then we can find $s \in \operatorname{ann}(M) \subset A, s \notin \mathfrak{p}$ and $M_{\mathfrak{p}}=0$, that is to say $\operatorname{supp}(M) \subseteq Z(\operatorname{ann}(M))$.

Conversely, if $M=A x_{1}+\ldots+A x_{r}$ is a finitely generated $A$-module and $\mathfrak{p} \in \operatorname{spec}(A)$ is such that $M_{\mathfrak{p}}=0$, then we can find $s_{i} \in A-\mathfrak{p}$ such that $s_{i} x_{i}=0$ for $i=1, \ldots, r$. We have $s=s_{1} \ldots s_{r} \in$ ann $(M)$ but $s \notin \mathfrak{p}$ and herefore $\mathfrak{p} \nsupseteq \operatorname{ann}(M)$, that is to say $Z(\operatorname{ann}(M)) \subseteq \operatorname{supp}(M)$.

Q.E.D.

We obtain at once the following corollary from the two preceding propositions:

Corollary 6.6: For any short exact sequence:

$$
0 \rightarrow M^{\prime} \stackrel{f}{\rightarrow} M \stackrel{g}{\rightarrow} M^{\prime \prime} \rightarrow 0
$$

one has the relation:

$$
\operatorname{supp}(M)=\operatorname{supp}\left(M^{\prime}\right) \cup \operatorname{supp}\left(M^{\prime \prime}\right) .
$$

Having in mind the preceding example, we now define poles and zeros for systems with coefficients in a field $k$ of constants. 
With $D=k[d]=k\left[d_{1}, \ldots, d_{n}\right]$ as usual, separating the control variables into inputs $u$ and outputs $y$, we may use the canonical injection $D u \subset D u+D y$, take the intersection of $D u$ with the submodule of equations in $D u+D y$ allowing to define $M$ and define by residue the differential input module $M_{i n}$. A similar procedure may be applied with $y$ in place of $u$ and leads to the differential output module $M_{\text {out }}$. We may then introduce the differential modules $M_{\text {in }}^{\prime}=M_{\text {in }}+t(M), M_{\text {out }}^{\prime}=M_{\text {out }}+t(M)$ and obtain the following commutative diagram of inclusions:

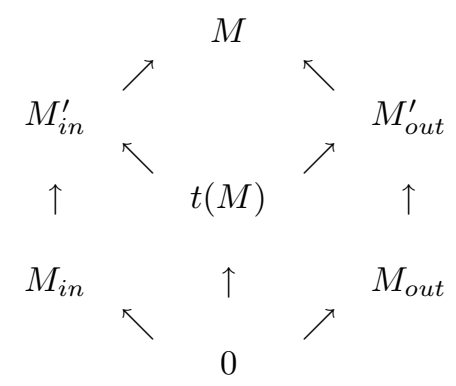

We now prove that all the known results on poles and zeros just amount to apply the preceding corollary to the various modules we have introduced, both with their sums and quotient whenever they can be defined.

For example, in order to define $\operatorname{supp}\left(M / M_{i n}\right)$, that provides the so-called system poles, we just need to add $u=0$ to the control OD equations and look for the annihilator of the differential module $M / M_{\text {in }}$ which is a torsion module by tradition in OD control. In the preceding example, we get the ideal $((s-1)(s-2))$ and the only two prime ideals containing it are $(s-1)$ and $(s-2)$. Now, the torsion submodule $t(M)$ is easily seen to be generated by $z=\dot{y}-2 y-\dot{u}$ satisfying $\dot{z}-z=0$. Hence, in order to define $\operatorname{supp}\left(M / M_{i n}^{\prime}\right)$, that provides the so-called controllable poles, we just need to add $u=0, \dot{y}-2 y-\dot{u}=0$ to the control OD equations and look for the annihilator of the differential module $M / m_{i n}^{\prime}$ which is generated by $(s-2)$ and is already prime. We have thus recovered the "poles" and could similarly recover the "zeros" by using now $\operatorname{supp}\left(M / M_{\text {out }}\right)$ and $\operatorname{supp}\left(M / M_{\text {out }}^{\prime}\right)$. Finally, in order to define $\operatorname{supp}(t(M))$, that provides the so-called input decoupling zeros, we have to look for the annihilator of $t(M)$ which is generated by $(s-1)$ and thus prime.

The only difficulty left is to define the corresponding concepts in the non-commutative case $D=K[d]$ when $K$ is an arbitrary differential field. For this, using the inclusion $D_{q}=\{P \in D \mid \operatorname{ord}(P) \leq q\} \subset D$, we obtain the inclusion $D_{q} y \subset D y$ inducing by residue, as above, a submodule $M_{q} \subset M$ which is not a differential module with an action $D_{r} M_{q} \subseteq M_{q+r}, \forall q, r \geq 0$ providing an equality for $q$ large enough. Looking at the composition $P, Q \in D \rightarrow P \circ Q \neq Q \circ P \in D$, we notice (exercise) that $\operatorname{gr}(D)=\oplus_{q=0}^{\infty} D_{q} / D_{q-1}$ is isomorphic to the commutative ring $K[\chi]=K\left[\chi_{1}, \ldots, \chi_{n}\right]$ of polynomials in $n$ indeterminates with coefficients in $K$. Introducing $G_{q}=M_{q} / M_{q-1}$ and setting $G=\operatorname{gr}(M)=\oplus_{q=0}^{\infty} G_{q}$, we notice that $G$ is a module over $\operatorname{gr}(D)$ and we are brought back to the commutative case with $\operatorname{gr}(D)$ and $G$ in place of $D$ and $M$. As a byproduct, we may state:

Definition 6.7: $\operatorname{char}(M)=\operatorname{supp}(G)$ is called the characteristic set of $M$ while ann $(G)$ is called the characteristic ideal.

Remark 6.8: According to the last corollary, one must rather use $\operatorname{rad}(\operatorname{ann}(G))$ in place of $\operatorname{ann}(G)$. (For more details, see $[10,19,20])$.

Remark 6.9: The above technique may also be used in order to define poles and zeros for non-linear systems through a generic linearization as will become clear from the following example.

Example 6.10: With $n=2, M=1, q=2$, let us consider the following non-linear system:

$$
y_{22}-\frac{1}{3}\left(y_{11}\right)^{3}=0, \quad y_{12}-\frac{1}{2}\left(y_{11}\right)^{2}=0
$$

We let the reader prove that this system is involutive with respective multiplicative variables $\left(x^{1}, x^{2}\right)$ and $\left(x^{1}\right)$. The generic linearization:

$$
Y_{22}-\left(y_{11}\right)^{2} Y_{11}=0, \quad Y_{12}-y_{11} Y_{11}=0
$$

is defined (exercise) over the differential field $K=\mathbb{Q}\left(y, y_{1}, y_{2}, y_{11}, y_{111}, \ldots\right)$ and the characteristic ideal in $K\left[\chi_{1}, \chi_{2}\right]$ becomes:

$$
\left(\left(\chi_{2}\right)^{2}-\left(y_{11}\right)^{2}\left(\chi_{1}\right)^{2}, \chi_{1}\left(\chi_{2}-y_{11} \chi_{1}\right)\right)
$$


Its radical is the prime ideal $\left(\chi_{2}-y_{11} \chi_{1}\right)$.

When $k$ is a ring of constants, the commutative ring $D=k\left[d_{1}, \ldots, d_{n}\right]$ of linear differential operators in $n$ commuting formal derivatives with constant coefficients in $k$ can be identified with the polynomial integral domain $A=k\left[\chi_{1}, \ldots, \chi_{n}\right]$ in $n$ indeterminates. In 1955, J.P. Serre conjectured that any projective module over $A$ was free, that is to say isomorphic to $A^{r}$ for some positive integer $r$ [9,23]. This result has been proved in 1976 , independently by D. Quillen and A.A. Suslin through very technical arguments and a constructive proof has only been given less than 10 years ago [15].

As a generalization of the above results, N.K. Bose and Z. Lin proposed in 1998 a conjecture on the possibility to factorize a certain type of full rank polynomial matrices through zero prime polynomial matrices in order to factor out the P.G.C.D. of the major minors [3]. Our purpose is to use algebraic analysis in order to solve positively this conjecture while giving its intrinsic module theoretic meaning.

Any linear operator $\mathcal{D}$ of order $q$ with constant coefficients acting on $m$ differential indeterminates $y^{1}, \ldots, y^{m}$ can be written as $\mathcal{D}=\sum_{0 \leq|\mu| \leq q} a^{\mu} d_{\mu}$ when using a multi-index $\mu=\left(\mu_{1}, \ldots, \mu_{n}\right)$ with $|\mu|=\mu_{1}+\ldots+\mu_{n}$ and $a^{\mu} \in k^{l \times m}$ for some $l$ while setting for short $d_{\mu}=\left(d_{1}\right)^{\mu_{1}} \ldots\left(d_{n}\right)^{\mu_{n}}$. According to what we said, $\mathcal{D}$ can be identified with the polynomial matrix $\varphi=\left(\sum a^{\mu} \chi_{\mu}\right) \in A^{l \times m}$. From now on, we shall suppose that $l \leq m$ and that $\varphi$ has $l$ rows, $m$ columns and maximal rank equal to $l$. In general, any operator $\mathcal{D}$ may have (generating) compatibility conditions expressed by another operator $\mathcal{D}_{1}$ such that $\mathcal{D}_{1} \circ \mathcal{D} \equiv 0$ and the above assumption just amounts to the fact that $\mathcal{D}_{1}$ is the zero operator and we shall say in this case that $\mathcal{D}$ is (formally) surjective.

Similarly, we may introduce the (formal) adjoint operator $\tilde{\mathcal{D}}=\operatorname{ad}(\mathcal{D})$ as usual. In actual practice, for a constant coefficient operator $\mathcal{D}$ with matrix $\varphi$, we shall identify the matrix of $\tilde{\mathcal{D}}$ with the simple transposed $\tilde{\varphi}$ of $\varphi$, forgetting about the need to also change $\chi$ to $-\chi$.

Example 6.11: When $n=3$, the curl operator is not surjective, is equal to its own adjoint and the action of the corresponding polynomial matrix just amounts to exterior product by the covector $\chi=\left(\chi_{1}, \ldots, \chi_{n}\right)$, up to sign. The div operator is surjective and its adjoint is the grad operator.

Let us introduce the module $M$ over $A$ defined by the finite presentation $A^{l} \stackrel{\varphi}{\longrightarrow} A^{m} \rightarrow M \rightarrow 0$ where $\varphi$ acts on the right on row vectors. Similarly, let $N$ be the module defined by the cokernel of $\tilde{\varphi}$. Following [], we set:

Definition 6.12: We denote by $I_{i}(\varphi)$ the ideal of $A$ generated by the determinants of the $i \times i$ submatrices (minors of size $i$ ) of $\varphi$, with the convention $I_{0}(\varphi)=A$. One can prove that the ideals $F i t t_{r}(M)=I_{m-r}(\varphi)$ of $A$, called $r$-Fitting ideal of $M$, only depend on $M$ and not on its presentation. Moreover we have $F_{i t t}(M)=F i t t_{r+l-m}(N)$.

If $M$ is any module over $A$, we denote by $a n n_{A}(M)$ or simply ann $(M)$ the annihilator of $M$ in $A$ and we shall set as usual $Z=$ zero and $M^{\star}=\operatorname{hom}_{A}(M, A)$. We quote the two main (very technical) results on Fitting ideals that will be crucially used in the sequel with $l \leq m$ and $r=m-l$ :

Theorem 6.13: $\operatorname{Fitt}_{0}(N) \subseteq \operatorname{ann}(N)$ and $\operatorname{rad}\left(F i t t_{0}(N)\right)=\operatorname{rad}(\operatorname{ann}(N))$.

Theorem 6.14: $M$ is a projective (thus free) module of rank $r$ if and only if Fitt $_{r}(M)=F i t t_{0}(N)=A$.

We now recall from section 3 the following three numbers:

- The last character $\alpha_{q}^{n}=r k_{D}(M) \leq m$.

- The number of nonzero characters $d(M)=d_{M}=d \leq n$.

- The smallest nonzero character $\alpha$.

These numbers are intrinsic because, when the corresponding system $R_{q}$ is involutive in the differential geometric framework, the Hilbert polynomial of $M$ is by definition:

$$
P_{M}(r)=\operatorname{dim}_{k}\left(R_{q+r}\right)=\frac{\alpha}{d !} r^{d}+\ldots
$$

It follows from the delicate Hilbert-Serre theorem $[10,19]$ that $d$ is also equal to the maximum dimension of the irreducible components of the characteristic set, this number being equal, in the present situation, to the maximum dimension of the irreducible components of the algebraic set defined by $\operatorname{ann}_{A}(M)$ as we are dealing with systems having constant coefficients. In particular, we have $d=n \Leftrightarrow \alpha_{q}^{n}=\alpha^{n} \neq 0$. We shall define the $\operatorname{codimension} c d(M)$ 
of $M$ to be $n-d$ and we may introduce the two following concepts that coincide when $n=1$ [20]:

Definition 6.15: $\varphi$ is said to be zero prime if $\operatorname{Fitt}_{r}(M)=$ Fitt $_{0}(N)=A$ or, equivalently, if $d(N)=-1$ (convention).

Definition 6.16: $\varphi$ is said to be minor prime if the elements of $F i t t_{0}(N)$ have no common divisor in $A \backslash k$ or, equivalently, if $d(N) \leq n-2$.

We are now ready to state the conjecture we want to prove:

Conjecture 6.17: Let us suppose that the greatest common divisor $c \in A$ of the $m ! /(m-l) ! l ! m i n o r s ~ a_{i}=c a_{i}^{\prime}$ of $\varphi$ is such that $\left(a_{1}^{\prime}, \ldots, a_{m ! /(m-l) ! l !}^{\prime}\right)=A$, then one can find $\varphi^{\prime} \in A^{l \times m}$ and $\psi \in A^{l \times l}$ such that $\varphi=\psi \circ \varphi^{\prime}$ and $\operatorname{det}(\psi)=c$.

Surprisingly, in order to understand the intrinsic meaning of this conjecture, we shall need many more (delicate) facts from algebraic analysis $[7,0,19,20]$. In particular, if $\ldots \stackrel{d_{2}}{\longrightarrow} F_{1} \stackrel{d_{1}}{\longrightarrow} F_{0} \stackrel{p}{\longrightarrow} M \rightarrow 0$ is a free resolution of the $A$-module $M$, we recall that the groups of cohomology of the dual complex $\ldots \stackrel{d_{2}^{\star}}{\longleftarrow} F_{1}^{\star} \stackrel{d_{1}^{\star}}{\longleftarrow} F_{0}^{\star} \leftarrow 0$, where $d^{\star}(f)=f \circ d, \forall f \in \operatorname{hom}_{A}(F, A)=F^{\star}$, do not depend on the resolution and will be denoted by $\operatorname{ext}_{A}^{i}(M, A)=$ $\operatorname{ker}\left(d_{i+1}^{\star}\right) / \operatorname{im}\left(d_{i}^{\star}\right)$ or simply by $\operatorname{ext}^{i}(M)$ with $\operatorname{ext}^{0}(M)=M^{\star}$. The proof of the three following results is quite delicate but valid for an arbitrary differential module $M$.

Theorem 6.18: $\operatorname{cd}\left(\operatorname{ext}^{i}(M)\right) \geq i$.

Theorem 6.19: $c d(M) \geq r \Longleftrightarrow \operatorname{ext}^{i}(M)=0, \forall i<r$.

Setting $\operatorname{char}(M)=Z(\operatorname{ann}(M))=\{\mathfrak{p} \in \operatorname{spec}(A) \mid \mathfrak{p} \supseteq \operatorname{ann}(M)\}$, we have:

Theorem 6.20: $\operatorname{char}(M)=\cup_{i=0}^{n} \operatorname{char}\left(\operatorname{ext}^{i}(M)\right)$.

We shall now use these results in order to give an intrinsic interpretation and solution of the previous conjecture.

Introducing the torsion submodule $t(M)=\{x \in M \mid \exists 0 \neq a \in A, a x=0\}$ and the torsion-free module $M^{\prime}=$ $M / t(M)$, the main trick will be to use $N$ and $N^{\prime}$ in the preceding theorems, in order to study $t(M), M$ and $M^{\prime}$. First of all, if $c \in k$, then $c \neq 0$ because of the maximum rank assumption on $\varphi$ and the conjecture is trivial, that is $M$ is projective, thus free. In particular, if $l=m$, then $M=0$ (See Janet conjecture in [20]). Hence, we may suppose that $c \in A \backslash k$. Surprisingly, in this case, we shall need all the previous results in order to prove that the quoted conjecture is equivalent to the following theorem:

Theorem 6.21: $M^{\prime}$ is a projective module if and only if $\operatorname{char}(N)$ is the union of irreducible varieties of the same dimension $n-1$.

Proof: If $M^{\prime}$ is projective, the kernel $K^{\prime}$ of the composition $A^{m} \rightarrow M \rightarrow M^{\prime}$ of epimorphisms is a projective module because the short exact sequence $0 \rightarrow K^{\prime} \rightarrow A^{m} \rightarrow M^{\prime} \rightarrow 0$ splits. It is thus a free module of rank $l$ because of the additivity property of the rank and the fact that, if $Q(A)$ is the quotient field of $A$, we have an isomorphism $Q(A) \otimes_{A} M \simeq Q(A) \otimes_{A} M^{\prime}$ of vector spaces over $Q(A)$. We obtain therefore a commutative and exact diagram:

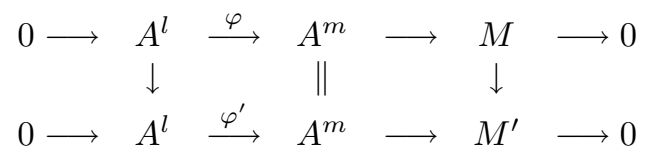

inducing the matrix $\psi: A^{l} \rightarrow A^{l}$ on the left, with $\varphi=\psi \circ \varphi^{\prime}$ acting on the right on row vectors. According to Theorem 2, we get $a_{i}=\operatorname{det}(\psi) a_{i}^{\prime}$ fot the corresponding minors and obtain the assumption of the conjecture with $c=\operatorname{det}(\psi)$. 
The hard step is the converse way. First of all, if $\operatorname{char}(N)$ is $(n-1)$-equidimensional, we find the assumption of the conjecture as we have indeed $\operatorname{ann}(N)=(c)$, though this monogenic ideal needs not be equal to its radical. Now, using Theorem 5.21 for $N$, we get:

$$
\operatorname{char}(N)=\operatorname{char}\left(\operatorname{ext}^{0}(N)\right) \cup \operatorname{char}\left(\operatorname{ext}^{1}(N)\right) \cup \operatorname{char}\left(\operatorname{ext}^{2}(N)\right) \cup \ldots
$$

Applying $\operatorname{hom}_{A}(\bullet, A)$ to the ker/coker exact sequece:

$$
0 \longleftarrow N \longleftarrow A^{l} \stackrel{\tilde{\varphi}}{\longleftarrow} A^{m} \longleftarrow M^{\star} \longleftarrow 0
$$

and using the fact that $\mathcal{D}$ surjective $\Leftrightarrow \varphi$ injective, we get $N^{\star}=e x t^{0}(N)=0$ and $\operatorname{char}(0)=\emptyset$.

It then follows from Section 5 that we have the $k e r / c o k e r$ exact sequence:

$$
0 \longrightarrow \operatorname{ext}^{1}(N) \longrightarrow M \stackrel{\epsilon}{\longrightarrow} M^{\star \star} \longrightarrow \operatorname{ext}^{2}(N) \longrightarrow 0
$$

where $\epsilon(x)(f)=f(x), \forall x \in M, \forall f \in M^{\star}$ and $e x t^{1}(N)=t(M)$, with $d(t(M))=n-1$ strictly in our case.

Using Theorem 3, we have $d\left(\operatorname{ext}^{i}(N)\right)<n-1$ when $i=2,3, \ldots, n$ and, from the assumption of the theorem, this is impossible unless $\operatorname{ext}^{i}(N)=0, \forall i=2,3, \ldots, n$.

Then, applying $\operatorname{hom}_{A}(\bullet, A)$ to the short exact sequence $0 \rightarrow t(M) \rightarrow M \rightarrow M^{\prime} \rightarrow 0$, we get $M^{\star}=M^{\prime \star}$ because, if $x \in t(M)$ is such that $a x=0$ with $a \neq 0$, we get $a f(x)=f(a x)=f(0)=0 \Rightarrow f(x)=0$ as $A$ is an integral domain and thus $t(M)^{\star}=0$.

Using the commutative and exact diagram:

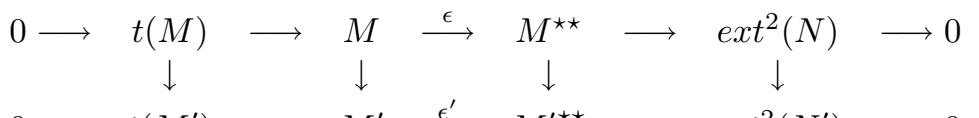

$$
\begin{aligned}
& 0 \longrightarrow t\left(M^{\prime}\right) \longrightarrow M^{\prime} \stackrel{\epsilon^{\prime}}{\longrightarrow} M^{\prime \star \star} \longrightarrow \operatorname{ext}^{2}\left(N^{\prime}\right) \longrightarrow 0
\end{aligned}
$$

where $t\left(M^{\prime}\right)=e x t^{1}\left(N^{\prime}\right)=0$ because $M^{\prime}$ is torsion-free, we obtain from an easy chase the isomorphism $e x t^{2}\left(N^{\prime}\right) \simeq$ $\operatorname{ext}^{2}(N)=0$.

Finally, from the previous ker/coker exact sequences defining $N$ and $N^{\prime}$, using twice the connecting sequence for the ext, we get:

$$
\operatorname{ext}^{i}\left(N^{\prime}\right) \simeq \operatorname{ext}^{i-2}\left(M^{\prime \star}\right) \simeq \operatorname{ext}^{i-2}\left(M^{\star}\right) \simeq \operatorname{ext}(N)=0, \forall i=3, \ldots, n
$$

It follows that $\operatorname{ext}^{i}\left(N^{\prime}\right)=0, \forall i \geq 1$ and $M^{\prime}$ is projective according to [20, Corollary 4].

Q.E.D.

Remark 6.22: This result is coherent with the fact that $N$ is defined up to an isomorphism (exercise) while $N^{\prime}$ is only defined up to a projective equivalence and $\operatorname{ext}^{i}(P)=0, \forall i \geq 1$ for any projective module $P$. It is also coherent with the long exact connecting sequence for ext :

$$
0 \rightarrow M^{\prime \star} \rightarrow M^{\star} \rightarrow 0 \rightarrow \operatorname{ext}^{1}\left(M^{\prime}\right) \rightarrow \operatorname{ext}^{1}(M) \rightarrow \operatorname{ext}^{1}(t(M)) \rightarrow \ldots
$$

as we deduce from it the monomorphism $0 \rightarrow N \rightarrow \operatorname{ext}^{1}\left(\operatorname{ext}^{1}(N)\right)$ showing that $N$ is 1-pure [19].

Remark 6.23: In a more specific way, taking into account Theorem 6.19 and applying it to $N$, we notice that:

$$
N^{*}=\operatorname{hom}_{D}(N, D)=\operatorname{ext}_{D}^{0}(N, D)=0 \Leftrightarrow \text { Ntorsion } \Leftrightarrow \mathcal{D} \text { formally surjective. }
$$

We obtain therefore the following recapitulating tabular for systems made up by differentially independent PD equations [22]:

\begin{tabular}{|l|l|l|l|}
\hline Module $M$ & $\operatorname{ext}_{D}^{i}(N, D)$ & $\boldsymbol{d}(\boldsymbol{N})$ & Primeness \\
\hline with torsion & $\operatorname{ext}_{D}^{0}(N, D)=0$ & $\leq n-1$ & $\emptyset$ \\
\hline torsion-free & $\operatorname{ext}_{D}^{i}(N, D)=0,0 \leq i \leq 1$ & $\leq n-2$ & minor prime \\
\hline reflexive & $\operatorname{ext}_{D}^{i}(N, D)=0,0 \leq i \leq 2$ & $\leq n-3$ & \\
\hline$\cdot$ & $\cdot$ & $\cdot$ &. \\
\hline$\cdot$ & $\cdot$ & $\cdot$ &. \\
\hline projective & $\operatorname{ext}_{D}^{i}(N, D)=0,0 \leq i \leq n$ & $\leq-1$ & zero prime \\
\hline
\end{tabular}


where we set $d(N)=-1$ when $\operatorname{char}(N)=\emptyset$. For example, the divergence operator for $n=3$ provides a good example of a differential module which is reflexive but not projective because $\operatorname{ext}_{D}^{3}(N, D) \neq 0$.

Example 6.24: With $n=3$ and $k=\mathbb{Q}$, let $M$ be defined by the two differentially independent PD equations $y_{12}^{3}-y_{3}^{2}-y^{3}=0, y_{22}^{3}-y_{3}^{1}=0$. We have $a_{1}=\left(\chi_{3}\right)^{2}, a_{2}=\chi_{3}\left(\chi_{1} \chi_{2}-1\right), a_{3}=\chi_{3}\left(\chi_{2}\right)^{2}$ and $b_{1}=\chi_{3}, b_{2}=$ $\chi_{1} \chi_{2}-1, b_{3}=\left(\chi_{2}\right)^{2}$ with $\left(\chi_{1}\right)^{2} b_{3}-\left(\chi_{1} \chi_{2}+1\right) b_{2}=1$, a result leading to $\operatorname{ann}(N)=\operatorname{rad}(\operatorname{ann}(N))=\left(\chi_{3}\right)$. The generating torsion element $z=y_{22}^{2}-y_{12}^{1}+y^{1}$ satisfies $z_{3}=0$. Though this is not evident at all, we may define $M^{\prime}$ by the two independent PD equations $y_{22}^{2}-y_{12}^{1}+y^{1}=0, y_{123}^{2}-y_{113}^{1}+y_{3}^{2}+y^{3}=0$ and we have the injective parametrization $u_{22}=y^{1}, u_{12}-u=y^{2}, u_{3}=y^{3}$ showing that $M^{\prime} \simeq A$ is free.

\section{I.7 CONCLUSION}

We hope to have convinced the reader that the results presented are striking enough to open a wide future for applications of computer algebra. The systematic use of the adjoint operator has allowed to relate together results as far from each other as the Quillen-Suslin theorem in module theory and the controllability criterion in control theory. A similar criterion for projective modules does exist and relies on the possibility to have finite length differential sequences [19]. We believe that the corresponding symbolic packages will be available in a short time. It will thus become possible to classify (differential) modules, having in mind that such a classification always describes hidden but deep concepts in the range of applications.

Exercises: We provide thereafter three exercises which can help the reader recapitulating the various concepts introduced through this chapter.

Exercise 1: In the motivating examples of Section 2, we have seen that the system:

$$
P y \equiv d_{22} y=u, \quad Q y \equiv d_{12} y-y=v
$$

where $P, Q \in D=\mathbb{Q}\left[d_{1}, d_{2}\right]$, admits the two generating compatibility conditions of order 4 :

$$
A \equiv d_{1122} u-u-d_{1222} v-d_{22} v=0, \quad B \equiv d_{1112} u-d_{11} u-d_{1122} v=0
$$

or the single compatibility condition of order 2 :

$$
C \equiv d_{12} u-u-d_{22} v=0
$$

1) Prove that the two systems $A=0, B=0$ on one side and $C=0$ on the other side for $(u, v)$ determine the same differential module $M \simeq D y=D$.

2) Determine the unique generating compatibility condition of order 2 satisfied by $(A$,$) .$

3) Exhibit the corresponding resolution (') of $M$ :

$$
0 \longrightarrow D \longrightarrow D^{2} \longrightarrow D^{2} \longrightarrow M \longrightarrow 0
$$

with central morphism $D A+D B \rightarrow D u+D v$.

4) Exhibit similarly a resolution (") of $M$ :

$$
0 \longrightarrow D \longrightarrow D^{2} \longrightarrow M \longrightarrow 0
$$

with morphism $D C \rightarrow D u+d v$.

5) Prove that (') projects onto (") by exhibiting an epimorphism $D^{2} \rightarrow D: D A+D B \rightarrow D C$.

6) Prove that the kernel of the preceding epimorphism is a free module by finding out an injective parametrization of the second order operator just obtained.

7) Find a lift for the preceding epimorphism and exhibit a short split exact sequence:

$$
0 \longrightarrow D \longrightarrow D^{2} \longrightarrow D \longrightarrow 0
$$

with morphism $D A+D B \rightarrow D C$ on the right.

8) Applying $\operatorname{hom}(\bullet, D)$ to the commutative diagram just obtained, prove that, if $N^{\prime}$ and $N^{\prime \prime}$ are the modules " $N$ " that can be constructed from the two preceding resolutions of $M$, then one has $N^{\prime} \simeq N^{\prime \prime} \oplus D$ and thus $\operatorname{ext}_{D}\left(N^{\prime}, D\right) \simeq \operatorname{ext}_{D}\left(N^{\prime \prime}, D\right)=0, \forall i \geq 1$. 
Exercise 2: With $m=2, n=2, q=1$, let us consider the following first order system with two variable coefficients, namely one arbitrary constant $\alpha$ and one arbitrary function $a(x)=a\left(x^{1}, x^{2}\right)$ (for example $\alpha \in \mathbb{Q}=k, a \in K=$ $\left.\mathbb{Q}\left(x^{1}, x^{2}\right)\right)$ :

$$
d_{2} y^{1}-\alpha d_{1} y^{1}-d_{2} y^{2}+a(x) y^{2}=0
$$

Study how the corresponding module $M$ does depend on the coefficients by exhibiting a "tree" of 4 possibilities, from the less generic to the most generic one (successively with torsion, free, torsion-free, projective).

1) In the case $M$ has torsion, exhibit a generator for the torsion submodule $t(M)$.

2) In the case $M$ is free, prove that $M \simeq D$.

3) In the case $M$ is torsion-free but not free, prove that $M \subset D$ with strict inclusion and prove that $M$ is not projective.

4) In the case $M$ is projective, exhibit a lift for the presentation $0 \rightarrow D \rightarrow D^{2} \rightarrow M \rightarrow 0$ which becomes therefore a split short exact sequence.

Exercise 3: With $n=1, \chi=\frac{d}{d t}$ and $D=\mathbb{Q}[d] \simeq \mathbb{Q}[\chi]$, we shall revisit the following example of a third order OD control system given in 1980 by Blomberg and Ylinen in [2]:

$$
\left(\begin{array}{ccc}
2 \chi^{3}+\chi^{2}-8 \chi-4 & 2 \chi+1 & 4 \chi^{2}+2 \chi \\
\chi^{2}-4 & \chi^{3}+5 \chi^{2}+8 \chi+5 & 3 \chi+2
\end{array}\right) \quad\left(\begin{array}{c}
y^{1} \\
y^{2} \\
u
\end{array}\right)=\left(\begin{array}{l}
0 \\
0
\end{array}\right)
$$

with one input $u$ and two outputs $\left(y^{1}, y^{2}\right)$.

1) Check that the above operator matrix $\mathcal{D}$ can be factorized through $\mathcal{D}=\mathcal{P} \circ \mathcal{D}^{\prime}$ with $\mathcal{P}$ square and $\mathcal{D}^{\prime}$ the operator matrix:

$$
\left(\begin{array}{ccc}
\chi^{2}-4 & 1 & 2 \chi \\
0 & \chi^{2}+3 \chi+2 & 1
\end{array}\right)
$$

Provide the corresponding commutative diagram interpretation, both in terms of operators and in terms of the differential modules $M$ and $M^{\prime}$ respectively determined by $\mathcal{D}$ and $\mathcal{D}^{\prime}$.

2) Prove that $M^{\prime}$ is torsion-free.

3) Check that the ideal generated in $\mathbb{Q}[\chi]$ by the 3 determinants of the $2 \times 2$ minors of $\mathcal{D}^{\prime}$ is equal to $\mathbb{Q}[\chi]$, that is this ideal contains 1 . Use this result in order to find out a lift for $\mathcal{D}^{\prime}$.

4) Prove that $M^{\prime} \simeq M / t(M)$.

5) Denoting by $M_{i n}$ the submodule of $M$ induced by the inclusion $D u \subset D u+D y^{1}+D y^{2}$ and introducing $M_{i n}{ }^{\prime}=M_{i n}+t(M) \subset M$, work out the ideals $\mathfrak{a}=\operatorname{ann}\left(M / M_{i n}{ }^{\prime}\right), \mathfrak{a}^{\prime}=\operatorname{ann}\left(M_{i n}{ }^{\prime} / M_{i n}\right), \mathfrak{a}^{\prime \prime}=\operatorname{ann}\left(M / M_{i n}{ }^{\prime}\right)$ and prove that $\operatorname{rad}(\mathfrak{a})=\operatorname{rad}\left(\mathfrak{a}^{\prime}\right) \cap \operatorname{rad}\left(\mathfrak{a}^{\prime \prime}\right)$ though $\mathfrak{a} \neq \mathfrak{a}^{\prime} \cap \mathfrak{a}^{\prime \prime}$.

For this last question, one may refer to the general theory by showing that $t(M) \cap M_{i n}=0 \Rightarrow M_{i n}{ }^{\prime} / M_{i n}=t(M)$ and exhibit the short exact sequence:

$$
0 \longrightarrow M_{i n}{ }^{\prime} / M_{i n} \longrightarrow M / M_{\text {in }} \longrightarrow M / M_{\text {in }}{ }^{\prime} \longrightarrow 0
$$

and finally find:

$$
\begin{gathered}
\mathfrak{a} \subset \operatorname{rad}(\mathfrak{a})=((2 \chi+1)(\chi+1)(\chi+2)(\chi-2)), \\
\mathfrak{a}^{\prime}=\operatorname{rad}\left(\mathfrak{a}^{\prime}\right)=((2 \chi+1)(\chi+2)), \\
\mathfrak{a}^{\prime \prime} \subset \operatorname{rad}\left(\mathfrak{a}^{\prime \prime}\right)=((\chi+1)(\chi+2)(\chi-2)) .
\end{gathered}
$$

\section{References:}

[1] E.ARANDA-BRICAIRE, C.H. MOOG, J.-B. POMMET, A Linear Algebraic Framework for Dynamics Feedback Linearization, IEEE Transactions on Automatic Control, 40, 1, 1995, 127-132.

[2] H. BLOMBERG, Y. YLINEN, Algebraic Theory for Multivariable Linear Systems, Academic Press, 1983.

[3] N.K. BOSE, Z. LIN, A generalization of Serre's conjecture and related issues, Linear Algebra and its Applications, 338, 2001, 125-138.

[4] D. EISENBUD, Commutative Algebra with a view towards Algebraic Geometry, Graduate Texts in Mathematics 150, Springer, 1995.

[5] M. JANET, Sur les Systèmes aux dérivées partielles, Journal de Math., 8, 3, 1920, 65-151. 
[6] E.R. KALMAN, Y.C. YO, K.S. NARENDA, Controllability of Linear Dynamical Systems, Contrib. Diff. Equations, 1, 2, 1963, 189-213.

[7] M. KASHIWARA, Algebraic Study of Systems of Partial Differential Equations, Mémoires de la Société Mathématique de France 63, 1995, (Transl. from Japanese of his 1970 Master's Thesis).

[8] E. KUNZ, Introduction to Commutative Algebra and Algebraic Geometry, Birkhäuser, 1985.

[9] T.Y. LAM, Serre's Conjecture, Lecture Notes in Mathezmatics 635, Springer, 1978.

[10] P. MAISONOBE, C. SABBAH, D-Modules Cohérents et Holonomes, Travaux en Cours, 45, Hermann, Paris, 1993.

[11] B. MALGRANGE, Cohomologie de Spencer (d'après Quillen), Sém. Math. Orsay, 1966.

[12] H. MATSUMURA, Commutative Ring Theory, Cambridge Studies in Advanced Mathematics 8, Cambridge University Press, 1986.

[13] U. OBERST, Multidimensional Constant Linear Systems, Acta Appl. Math., 20, 1990, 1-175.

[14] V.P. PALAMODOV, Linear Differential Operators with Constant Coefficients, Grundlehren der Mathematischen Wissenschaften 168, Springer, 1970.

[15] H. PARK, C. WOODBURN, An Algorithmic Proof of Suslin's Stability Theorem for Polynomial Rings, J. Algebra, 178, 1995, 277-298.

[16] J.F. POMMARET, Géométrie Différentielle Algébrique et Théorie du Contrôle, C. R. Acad. Sci. Paris, 302, série I, 1986, 547-550.

[17] J.F. POMMARET, Dualité Différentielle et Applications, C. R. Acad. Sci. Paris, 320, Série I, 1995, 12251230.

[18] J.F. POMMARET, Partial Differential Equations and Group Theory: New Perspectives for Applications, Kluwer, 1994.

[19] J.F. POMMARET, Partial Differential Control Theory, Kluwer, 2001.

(http://cermics.enpc.fr/ pommaret/home.html)

[20] J.F. POMMARET, A. QUADRAT, Algebraic Analysis of Linear Multidimensional Control Systems, IMA Journal of Mathematical Control and Informations, 16, 1999, 275-297.

[21] J.F. POMMARET, A. QUADRAT, Localization and Parametrization of Linear Multidimensional Control Systems, Systems and Control Letters, 37, 1999, 247-260.

[22] A. QUADRAT, Analyse Algébrique des Systèmes de Contrôle Linéaires Multidimensionnels, Thèse de Docteur de l'Ecole Nationale des Ponts et Chaussées, 1999(http://www-sop.inria.fr/cafe/Alban.Quadrat/ index.html).

[23] J.J. ROTMAN, An Introduction to Homological Algebra, Pure and Applied Mathematics, Academic Press, 1979 .

[24] D.C. SPENCER, Overdetermined Systems of Partial Differential Equations, Bull. Amer. Math. Soc., 75, $1965,1-114$.

[25] W.M. WONHAM, Linear Multivariable Control: a Geometric Approach, Springer, 1985.

[26] J. WOOD, Modules and behaviours in nD systems theory, Multidimensional Systems and Signal Processing, $11,2000,11-48$.

[27] E. ZERZ, Topics in Multidimensional Linear Systems Theory, Lecture Notes in Control and Information Sciences 256, Springer, 2000. 